\title{
The MAGNUM survey: different gas properties in the outflowing and disc components in nearby active galaxies with MUSE`
}

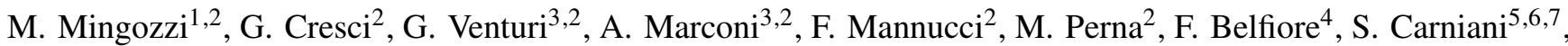 \\ B. Balmaverde ${ }^{8}$, M. Brusa ${ }^{1,9}$, C. Cicone ${ }^{8}$, C. Feruglio ${ }^{10}$, A. Gallazzi ${ }^{2}$, V. Mainieri ${ }^{11}$, R. Maiolino ${ }^{5,6}$, T. Nagao ${ }^{12}$, \\ E. Nardini ${ }^{2}$, E. Sani ${ }^{13}$, P. Tozzi ${ }^{2}$, and S. Zibetti ${ }^{2}$
}

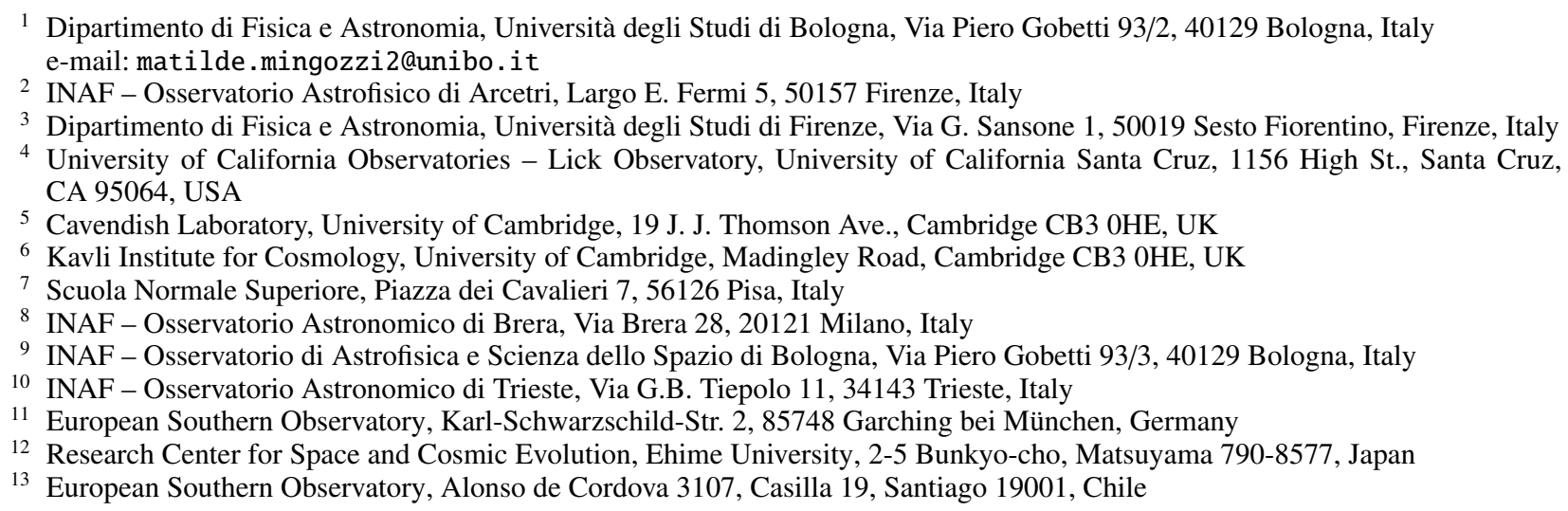

Received 3 October 2018 / Accepted 16 November 2018

\begin{abstract}
We investigated the interstellar medium (ISM) properties of the disc and outflowing gas in the central regions of nine nearby Seyfert galaxies, all characterised by prominent conical or biconical outflows. These objects are part of the Measuring Active Galactic Nuclei Under MUSE Microscope (MAGNUM) survey, which aims to probe their physical conditions and ionisation mechanism by exploiting the unprecedented sensitivity of the Multi Unit Spectroscopic Explorer (MUSE), combined with its spatial and spectral coverage. Specifically, we studied the different properties of the gas in the disc and in the outflow with spatially and kinematically resolved maps by dividing the strongest emission lines in velocity bins. We associated the core of the lines with the disc, consistent with the stellar velocity, and the redshifted and the blueshifted wings with the outflow. We measured the reddening, density, ionisation parameter, and dominant ionisation source of the emitting gas for both components in each galaxy. We find that the outflowing gas is characterised by higher values of density and ionisation parameter than the disc, which presents a higher dust extinction. Moreover, we distinguish high- and low-ionisation regions across the portion of spatially resolved narrow-line region (NLR) traced by the outflowing gas. The high-ionisation regions characterised by the lowest $\left[\mathrm{N}_{\mathrm{II}}\right] / \mathrm{H} \alpha$ and $\left[\mathrm{S}_{\mathrm{II}}\right] / \mathrm{H} \alpha$ line ratios generally trace the innermost parts along the axis of the emitting cones where the $\left[\mathrm{S}_{\mathrm{III}}\right] /\left[\mathrm{S}_{\mathrm{II}}\right]$ line ratio is enhanced, while the low-ionisation regions follow the cone edges and/or the regions perpendicular to the axis of the outflows, also characterised by a higher [O III] velocity dispersion. A possible scenario to explain these features relies on the presence of two distinct populations of line emitting clouds: one is optically thin to the radiation and is characterised by the highest excitation, while the other is optically thick and is impinged by a filtered, and thus harder, radiation field which generates strong low-excitation lines. The highest values of $\left[\mathrm{N}_{\mathrm{II}}\right] / \mathrm{H} \alpha$ and $\left[\mathrm{S}_{\mathrm{II}}\right] / \mathrm{H} \alpha$ line ratios may be due to shocks and/or a hard filtered radiation field from the active galactic nucleus.
\end{abstract}

Key words. galaxies: ISM - galaxies: Seyfert - galaxies: jets

\section{Introduction}

The gas located in the inner kiloparsecs of active galaxies plays a crucial role in many important processes; it fuels accretion onto the central black hole (BH) in active galactic nuclei (AGN) and absorbs the energy and momentum injected in the interstellar medium (ISM) by the AGN, becoming a key ingredient in the $\mathrm{BH}-$ galaxy co-evolution. The AGN radiation acts as a flashlight

\footnotetext{
* The maps of the derived quantities shown in the Appendix (FITS files) are only available at the CDS via anonymous ftp to cdsarc.u-strasbg.fr $(130.79 .128 .5)$ or via http://cdsarc. u-strasbg.fr/viz-bin/qcat?]/A+A/622/A146
}

that illuminates and ionises the nearby gas, forming the narrowline region (NLR). Outflows powered by accretion disc winds and relativistic jets interact with the NLR gas, possibly inducing feedback effects on the host galaxy (Cresci \& Maiolino 2018). Feedback mechanisms appear necessary to explain the presentday scaling relations between the host galaxy properties and $\mathrm{BH}$ mass and prevent galaxies from overgrowing (e.g. Silk \& Rees 1998; Fabian 2012).

The NLR can extend by several kpc in Seyfert galaxies (i.e. extended narrow-line regions, ENLRs, e.g. Greene et al. 2012; Sun et al. 2017, 2018) and has a relatively low-density $\left(n_{\mathrm{e}} \sim 10^{2}-10^{4} \mathrm{~cm}^{-3}\right.$, e.g. Vaona et al. 2012). It comprises 
ionised and neutral gas set-up in clouds, emitting low- and highionisation lines, such as [S II] $\lambda 6717,31$ and [O III] $\lambda \lambda 44959,5007$, respectively, of elements photoionised by the non-stellar continuum emission of the AGN (e.g. Netzer 2015). These clouds are often observed to be entrained in AGN-driven outflows (e.g. Hutchings et al. 1998; Crenshaw \& Kraemer 2000) that, especially in nearby Type 2 AGN, are observed to be fan-shaped, showing clearly defined biconical structures (e.g. Storchi-Bergmann et al. 1992; Das et al. 2006; Crenshaw et al. 2010; Cresci et al. 2015a; Venturi et al. 2018). Therefore, resolved NLRs are ideal laboratories for investigating in detail the effects of feeding and feedback, and for constraining the ionisation structure of the emitting regions.

Specifically, diagnostic diagrams constructed from emissionline intensity ratios are widely used to differentiate between excitation mechanisms. Firstly, Baldwin et al. (1981) proposed the $[\mathrm{N} \mathrm{II}] / \mathrm{H} \alpha$ versus $[\mathrm{O} \mathrm{III}] / \mathrm{H} \beta$ diagram in order to discriminate $\mathrm{H}$ II-like sources from objects photoionised by a harder radiation field, such as a power-law continuum by an AGN or shock excitation. This classification scheme was enlarged and improved by Veilleux \& Osterbrock (1987), who added the $\left[\mathrm{S}_{\text {II }}\right] / \mathrm{H} \alpha$ and the $\left[\mathrm{O}_{\mathrm{I}}\right] / \mathrm{H} \alpha$ line ratios. These are known as Baldwin-PhillipsTerlevich (BPT) diagrams and involve line ratios that are not significantly separated in wavelength, minimising the effects of differential reddening by dust, and can discriminate among three different groups of emission line galaxies: those excited by starbursts, the Seyfert galaxies, and those found in the lowionisation (nuclear) emission-line regions (LI(N)ERs, Heckman 1980; Belfiore et al. 2016).

There have been many studies aimed at identifying the ionisation source in Seyfert and star-forming galaxies through the observed intensity ratios. Photoionisation models have had remarkable success in reproducing high-ionisation AGN lines, such as [O III] and [S III] (Ferguson et al. 1997; Komossa \& Schulz 1997), while the excitation mechanisms for low-ionisation lines (e.g. $\left[\mathrm{N}_{\mathrm{II}}\right],\left[\mathrm{S}_{\mathrm{II}}\right],\left[\mathrm{O}_{\mathrm{I}}\right]$ ) in the region overlapping with star-forming galaxies remains uncertain. Furthermore, fast radiative shocks produced by different phenomena (e.g. cloud-cloud collisions, the expansion of $\mathrm{H}$ II regions into the surrounding ISM, outflows from young stellar objects, supernova blast waves, outflows from active and starburst galaxies, and collisions between galaxies) can be a powerful ionising source and can provide an important component of the total energy budget, and in some circumstances dominate the line emission spectrum (Dopita \& Sutherland 1995, 1996; Allen et al. 2008).

The recent development of powerful integral field unit (IFU) spectrographs has allowed us to make a considerable step forward in understanding galaxy evolution, providing spatially resolved information on the kinematics of the gas and stars, and on galaxy properties, in terms of gas ionisation and chemical abundances (e.g. the CALIFA, Sánchez et al. 2012; SAMI, Allen et al. 2015; and MaNGA, Bundy et al. 2015 surveys, locally, and Förster Schreiber et al. 2009; Law et al. 2009; Cresci et al. 2010; Contini et al. 2012; Wisnioski et al. 2015; Stott et al. 2016; Turner et al. 2017 at high redshift). Integral field spectroscopy (IFS) is a powerful tool also for the study of outflows, both in the local Universe (e.g. Storchi-Bergmann et al. 2010; Sharp \& Bland-Hawthorn 2010; Riffel et al. 2013; McElroy et al. 2015; Cresci et al. 2015a; Karouzos et al. 2016a,b) and at higher redshift (e.g. Cano-Díaz et al. 2012; Liu et al. 2013; Cresci et al. 2015b; Perna et al. 2015; Harrison et al. 2016; Carniani et al. 2016; Förster Schreiber et al. 2018a). Integral field data can indeed shed light on the impact of AGN activity on the host galaxy through the kinematic signatures visible in emission-line profiles and the spatial distribution of emission-line flux ratios.

In this paper, we present our results obtained with the survey Measuring Active Galactic Nuclei Under MUSE Microscope (MAGNUM, PI Marconi, Cresci et al. 2015a; Venturi et al. 2017, 2018, and in prep.) survey, which studies nearby AGN $\left(D_{\mathrm{L}}<50 \mathrm{Mpc}\right)$ by probing the physical conditions of the NLRs/ENLRs, and also studies the interplay between nuclear activity and star formation (SF), and the properties of outflows thanks to the unprecedented combination of spatial and spectral coverage provided by the integral field spectrograph MUSE at VLT (Bacon et al. 2010).

Specifically, in this paper we use IFS to conduct detailed studies on how ISM properties (e.g. density, temperature, ionisation, reddening) and the relative contributions of different ionisation mechanisms vary within individual galaxies, all characterised by prominent conical or biconical outflows. Taking a step forward, we kinematically disentangle the disc and outflow components, and compare their gas properties.

The paper is structured as follows. In Sect. 2 we introduce the galaxy sample and the data analysis, while in Sect. 3 we explain our approach to disentangle the outflow component from the disc, and we investigate the gas properties through proper diagnostics and the gas excitation through BPT diagrams. Finally, in Sect. 4 we discuss our results, making a comparison with photoionisation and shock models found in the literature, and in Sect. 5 we conclude with a summary of the results.

\section{Galaxy sample}

MAGNUM galaxies have been selected by cross-matching the optically selected AGN samples of Maiolino \& Rieke (1995) and Risaliti et al. (1999), and Swift-BAT 70-month Hard Xray Survey (Baumgartner et al. 2013), choosing only sources observable from Paranal Observatory $\left(-70^{\circ}<\delta<20^{\circ}\right)$ and with a luminosity distance $D_{\mathrm{L}}<50 \mathrm{Mpc}$. In Venturi et al. (in prep.), we present our sample, explaining in detail the selection criteria, data reduction, and analysis, and investigating the kinematics of the ionised gas. In this work we present our results for nine Seyfert galaxies, namely Centaurus A, Circinus, NGC 4945, NGC 1068, NGC 1365, NGC 1386, NGC 2992, NGC 4945, and NGC 5643. In the following, we briefly describe their main features.

\subsection{MAGNUM galaxies}

Centaurus $A$ (Seyfert $2-D_{\mathrm{L}} \sim 3.82 \mathrm{Mpc}-1^{\prime \prime} \sim 18.5 \mathrm{pc}$ ) is an early-type galaxy characterised by a major axis stellar component, a minor axis dust-lane, and a gas disc. The last follows the dust-lane and comprises the majority of the observed ionised, atomic, neutral, and molecular gas (Morganti 2010). It is characterised by the presence of a spectacular double-sided radio jet revealed in both the radio and X-rays (e.g. Blanco et al. 1975; Hardcastle et al. 2003), which was found to match a diffuse highly ionised halo of [O III] (Kraft et al. 2008) first identified by Bland-Hawthorn \& Kedziora-Chudczer (2003).

Circinus (Seyfert $2-D_{\mathrm{L}} \sim 4.2 \mathrm{Mpc}-1^{\prime \prime} \sim 20.4 \mathrm{pc}$ ) is a nearby gas rich spiral, hosting one of the nearest Seyfert 2 nuclei known. The Seyfert 2 nature of Circinus is supported by the optical images showing a spectacular one-sided and wide-angled kpcscale [O III] cone, first revealed by Marconi et al. (1994; see also Veilleux \& Bland-Hawthorn 1997), and the optical/IR spectra 
rich in prominent and narrow coronal lines (Oliva et al. 1994; Moorwood et al. 1996). Furthermore, from the X-ray spectral analysis Circinus is a highly obscured AGN, but with strong thermal dust emission dominated by heating from nuclear star formation (Matt et al. 2000).

IC 5063 (Seyfert $2-D_{\mathrm{L}} \sim 45.3 \mathrm{Mpc}-1^{\prime \prime} \sim 219.6 \mathrm{pc}$ ) is a massive early-type galaxy with a central gas disc and a radio jet that propagates for several hundred parsec before it extends beyond the disc plane (Morganti et al. 1998). An extended ( $20 \mathrm{kpc})$ [O III]-dominated double ionisation cone with an $\mathrm{X}$ morphology was observed (Colina et al. 1991), ubiquitously dominated by photoionisation from the AGN (Sharp \& Bland-Hawthorn 2010). In this galaxy there is one of the most spectacular examples of jet-driven outflow, with similar features in the ionised, neutral atomic, and molecular phase, since the radio plasma jet is expanding into a clumpy gaseous medium, possibly creating a cocoon of shocked gas which is pushed away from the jet axis (Morganti et al. 1998; Sharp \& Bland-Hawthorn 2010; Tadhunter et al. 2014; Dasyra et al. 2015; Oosterloo et al. 2017).

NGC 1068 (Seyfert $2-D_{\mathrm{L}} \sim 12.5 \mathrm{Mpc}-1^{\prime \prime} \sim$ $60 \mathrm{pc}$ ) is one of the closest and archetypal Seyfert 2 galaxies. This galaxy also hosts powerful starburst activity (e.g. Lester et al. 1987; Bruhweiler et al. 1991) and is characterised by a large-scale oval and a nuclear stellar bar aligned NESW (Scoville et al. 1988; Thronson et al. 1989). NGC 1068 is known to host a radio jet, observed from X-ray to radio wavelengths (Bland-Hawthorn et al. 1997). Its activity seems confined to bipolar cones centred on the AGN, intersecting the plane of the disc with an inclination of $\sim 45^{\circ}$, such that the disc interstellar medium to the $\mathrm{NE}$ and $\mathrm{SW}$ sees the nuclear radiation field directly (Cecil et al. 1990; Gallimore et al. 1994). This galaxy shows clear evidence of outflowing material in both the ionised (e.g. Macchetto et al. 1994; Axon et al. 1997; Cecil et al. 2002; Barbosa et al. 2014) and molecular gas (García-Burillo et al. 2014; Gallimore et al. 2016), possibly driven by the radio jet (Cecil et al. 1990; Gallimore et al. 1994; Axon et al. 1997), located in the north-east and south-west directions.

NGC 1365 (Seyfert $1.8-D_{\mathrm{L}} \sim 18.6 \mathrm{Mpc}-1^{\prime \prime} \sim 90.2 \mathrm{pc}$ ) is a local spiral, characterised by a strong bar and prominent spiral structure, displaying nuclear activity and ongoing SF. A comprehensive review about the early works on this object is given by Lindblad (1999). Our MUSE data was already published by Venturi et al. (2018), who studied the circumnuclear gas in its different phases by comparing MUSE and Chandra data and analysing the nuclear and extended gas outflows. Specifically, Venturi et al. (2018) revealed that [O III] emission that traces the kpc-scale biconical outflow, ionised by the AGN, is nicely matched with the soft X-rays. However, the hard X-ray emission from the star-forming circumnuclear ring suggests that SF might in principle contribute to the outflow.

NGC 1386 (Seyfert $2-D_{\mathrm{L}} \sim 15.6 \mathrm{Mpc}-1^{\prime \prime} \sim 76 \mathrm{pc}$ ), classified as a $\mathrm{Sb} / \mathrm{c}$ spiral galaxy, is one of nearest Seyfert 2 galaxies and has an inclination of $\sim 65^{\circ}$ with respect to the line of sight. Lena et al. (2015) analysed the gas and stellar kinematics in the inner regions $(530 \times 680 \mathrm{pc})$, obtained from IFS GEMINI South telescope observations, revealing the presence of a bright nuclear component, two elongated structures to the north and south of the nucleus, and low-level emission extending over the whole field of view (FOV), probably photoionised by the AGN. Furthermore, Lena et al. (2015) identified a bipolar outflow aligned with the radiation cone axis. This fea- ture is resolved in HST observations (Ferruit et al. 2000) into two bright knots that represent the approaching and receding sides of the outflow, respectively. Finally, Lena et al. (2015) suggested the presence of an outflow and/or rotation in a plane roughly perpendicular to the AGN radiation cone axis, extending $2^{\prime \prime}-3^{\prime \prime}$ on both sides of the nucleus, characterised by an enhanced velocity dispersion and coincident with a faint, barlike emission structure revealed by HST imaging (Ferruit et al. 2000).

NGC 2992 (Seyfert $1.9-D_{\mathrm{L}} \sim 31.5 \mathrm{Mpc}-1^{\prime \prime} \sim 150 \mathrm{pc}$ ) is a spiral galaxy hosting a Seyfert nucleus and interacting with the companion galaxy NGC 2993, located to the south-east. This galaxy is classified as a Seyfert 1.9 in the optical, on the basis of a broad $\mathrm{H} \alpha$ component with no corresponding $\mathrm{H} \beta$ component in its nuclear spectrum, although its type has changed between 1.5 and 2 according to past observations (Trippe et al. 2008). Looking at the optical image of this galaxy, a prominent dust lane extending along the major axis of the galaxy and crossing the nucleus stands out (Ward et al. 1980). Opposite ENLR cones extend along either side of the disc with large opening angles, and are both easily visible due to the high inclination of NGC 2992 (e.g. Allen et al. 1999). Evidence of a wide biconical large-scale outflow, extending above and below the plane of the galaxy and possibly driven by AGN activity (Friedrich et al. 2010), comes from $\mathrm{H} \alpha$ and [O III] emission and soft X-ray observations (e.g. Colina et al. 1987; Colbert et al. 1996a,b; Veilleux et al. 2001).

NGC 4945 (Seyfert $2-D_{\mathrm{L}} \sim 3.7 \mathrm{Mpc}-1^{\prime \prime} \sim 18 \mathrm{pc}$ ) is an almost perfectly edge-on spiral galaxy, well known for being one of the closest objects where AGN and starburst activity coexist. Its central region is characterised by a very strong obscuration, associated with a prominent dust lane aligned along the major axis of the galactic disc, revealed by near- and mid-infrared spectroscopy typical of highly obscured ultra-luminous infrared galaxies (ULIRGs; Pérez-Beaupuits et al. 2011). This galaxy is characterised by a biconical outflow, clearly visible from the [N II] emission map shown in Venturi et al. (2017), where the NW lobe is far brighter than the SE lobe, which is observed only at $15^{\prime \prime}$ from the centre, likely being completely dust-obscured at smaller radii. The AGN presence is only supported by X-rays observations (e.g. Guainazzi et al. 2000), meaning that either its UV radiation is totally obscured along all lines of sight or that it lacks UV photons with respect to X-rays, implying in both cases a non-standard activity (Marconi et al. 2000).

NGC 5643 (Seyfert $2-D_{\mathrm{L}} \sim 17.3 \mathrm{Mpc}-1^{\prime \prime} \sim 84 \mathrm{pc}$ ) is a barred almost face-on Seyfert 2. This galaxy is characterised by a wellknown ionisation cone extending eastward of the nucleus and parallel to the bar (e.g. Schmitt et al. 1994; Simpson et al. 1997; Fischer et al. 2013), and by a sharp, straight dust lane extended from the sides of the central nucleus out to the end of the bar, roughly parallel to its major axis and clearly visible to the east of the nucleus (Ho et al. 2011). Our MUSE data of this target has been already published by Cresci et al. (2015a), who analysed the double-sided ionisation cone, revealed as a blueshifted, asymmetric wing of the [O III] emission line, up to a projected velocity of $\sim-450 \mathrm{~km} \mathrm{~s}^{-1}$, parallel to the low-luminosity radio and X-ray jet, and possibly collimated by a dusty structure surrounding the nucleus. Furthermore, Cresci et al. (2015a) found signs of positive feedback triggered by the outflowing gas in two star-forming clumps, located at the edge of the dust lane of the bar (see Silk 2013), and also characterised by a faint $\mathrm{CO}(2-1)$ emission (Alonso-Herrero et al. 2018). 


\subsection{Data analysis}

The data reduction was performed using the MUSE pipeline (v1.6). The final datacubes consist of $\sim 300 \times 300$ spaxels, for a total of over 90000 spectra with a spatial sampling of $0.2^{\prime \prime} \times 0.2^{\prime \prime}$ and a spectral resolution going from 1750 at $4650 \AA$ to 3750 at $9300 \AA$. The FOV of $1^{\prime} \times 1^{\prime}$ covers the central part of the galaxies, spanning from 1 to $10 \mathrm{kpc}$, according to their distance. The average seeing of the observations, derived directly from foreground stars in the final datacubes, is $\sim 0.8^{\prime \prime}$. Here we summarise the main steps of the data analysis, while full details are given in the survey presentation paper by Venturi et al. (in prep.).

The datacubes were analysed making use of a set of custom python scripts in order to subtract the stellar continuum and fit the emission lines with multiple Gaussian components where needed. First of all, the stellar continuum was modelled using a linear combination of Vazdekis et al. (2010) synthetic spectral energy distributions for single stellar population models in the wavelength range $4750-7500 \AA$. In order to subtract the continuum around the $\left[\mathrm{S}_{\mathrm{III}}\right] \lambda 9069$, we performed a polynomial fit to the local continuum since this line is not contaminated by underlying absorptions. We applied a Voronoi tessellation (Cappellari \& Copin 2003) in order to achieve an average signalto-noise ratio $(\mathrm{S} / \mathrm{N})$ per wavelength channel of 50 on the continuum under $5530 \AA$, we performed the continuum fit using the Penalized Pixel-Fitting (pPXF; Cappellari \& Emsellem 2004) code on the binned spaxels, to reproduce the systemic velocity and the velocity dispersion of the stellar absorption lines. To do this, we simultaneously fit the main emission lines included in the selected wavelength range $(\mathrm{H} \beta-[\mathrm{O}$ III $] \lambda \lambda 4959,5007-$ [O I $\left.] \lambda \lambda 6300,64-\mathrm{H} \alpha-\left[\mathrm{N}_{\text {II }}\right] \lambda \lambda 6548,84-\left[\mathrm{S}_{\mathrm{II}}\right] \lambda \lambda 6717,31\right)$ in order to better constrain the absorption underlying the Balmer lines. Fainter lines and regions affected by sky residuals were masked. Then, the fitted stellar continuum in each Voronoi bin was subtracted spaxel by spaxel. Since NGC 1365 and NGC 2992 host a Seyfert 1 nucleus, we made use of an additional template when fitting the very central bins in order to simultaneously reproduce the broad-line region (BLR) features (broad Balmer lines and $\mathrm{Fe}$ II forest) and the $\mathrm{BH}$ accretion disc continuum.

From the continuum-subtracted cube, we generated both a spatially smoothed data cube and a Voronoi-binned data cube. The former is obtained by spatially smoothing the cube with a Gaussian kernel having $\sigma=1$ spaxel (i.e. $0.2^{\prime \prime}$ ), without degrading significantly the spatial resolution, given the observational seeing. The latter, produced by applying a Voronoi binning in the range $\sim 4850-4870 \AA$ in the vicinity of $\mathrm{H} \beta$ and requiring an average $\mathrm{S} / \mathrm{N}$ per wavelength channel of at least 3 , is used to make comparisons among the galaxies (see Sect. 4). Finally, in each spaxel, we fit the gas emission lines in the same spectral range used for the stellar fitting, making use of MPFIT (Markwardt 2009), with one, two, three, or four Gaussians according to the peculiarities of the line profile, tying the velocity and widths of each component to be the same for all the lines and leaving the intensities free to vary, apart from the [O III], [O I $]$, and [N II] doublets, where an intrinsic ratio of 0.333 between the two lines was used. We applied a reduced $\chi^{2}$ selection to the different fits made in each spaxel in order to define where multiple components were really needed to reproduce the observed spectral profiles, with the idea of keeping the number of fit parameters as low as possible and using multiple components only in case of complex non-Gaussian line profiles (for further details see Venturi et al., in prep.). This happens mainly in the central parts of the galaxies and in the outflowing cones.

\section{Gas properties: disc versus outflow}

Excitation and ionisation conditions, and dust attenuation of the ISM in each MAGNUM galaxy can be investigated through specific emission-line ratios. Thanks to IFS, we explore the different properties of the disc and the outflow of our galaxies by separating and analysing the different gas components across the MUSE FOV.

Specifically, we define velocity channels of $\sim 50 \mathrm{~km} \mathrm{~s}^{-1}$, ranging from $-1000 \mathrm{~km} \mathrm{~s}^{-1}$ to $+1000 \mathrm{~km} \mathrm{~s}^{-1}$ around the main emission lines $\left(\mathrm{H} \beta-\left[\mathrm{O}_{\mathrm{III}}\right]-\left[\mathrm{O}_{\mathrm{I}}\right]-\mathrm{H} \alpha-[\mathrm{N}\right.$ II $\left.]-\left[\mathrm{S}_{\mathrm{II}}\right]-\left[\mathrm{S}_{\mathrm{III}}\right]\right)$, centring the zero velocity to the measured stellar velocity in each spaxel of the MUSE FOV for all the galaxies since the stellar velocity is generally a good approximation of the gas velocity in the disc. In order to derive the flux in each velocity bin, we integrate the fitted line profile, performed as described in Sect. 2.2, within each velocity channel of the emission lines taken into account. However, Centaurus A is characterised by a strong misalignment between stars and gas (e.g. Morganti 2010), and thus we consider the global systemic velocity $\left(v_{\text {sys }}=547 \mathrm{~km} \mathrm{~s}^{-1}\right)$ as a reference for the gas disc velocity.

We associate the velocity channels close to the core of the lines in the fitted line profile with the disc (hereafter disc component), and the sum of the redshifted and blueshifted channels with the outflow (hereafter outflow component). Specifically, the disc component comprises velocity in the range $-100 \mathrm{~km} \mathrm{~s}^{-1}<$ $v<100 \mathrm{~km} \mathrm{~s}^{-1}$ for Circinus, NGC 1365, and NGC 1386, and $-150 \mathrm{~km} \mathrm{~s}^{-1}<v<150 \mathrm{~km} \mathrm{~s}^{-1}$ for the other galaxies, while the outflow component is associated with velocities $v>+150 \mathrm{~km} \mathrm{~s}^{-1}$ and $v<-150 \mathrm{~km} \mathrm{~s}^{-1}$ for Circinus, NGC 1365 and NGC 1386, $v>+250 \mathrm{~km} \mathrm{~s}^{-1}$ and $v<-250 \mathrm{~km} \mathrm{~s}^{-1}$ for NGC 1068, and $v>+200 \mathrm{~km} \mathrm{~s}^{-1}$ and $v<-200 \mathrm{~km} \mathrm{~s}^{-1}$ for the other galaxies. These values are chosen after an accurate spaxel-by-spaxel analysis of the spectra in the outflowing regions of the galaxies. Overall, the disc component represents the low-velocity component of the ionised gas, which is rotating similarly to the stars, while the outflow component (i.e. the high-velocity component) is moving faster than the stellar velocity, and is partly blueshifted and partly redshifted with respect to it. Since we are looking at the central regions of AGN galaxies, the NLR is present in both the disc and in the outflow components.

Figure 1 shows a single spaxel spectrum extracted from the Circinus galaxy datacube to exemplify our division between disc (in green with stripes) and outflow (in red) components for the [O III] $] \lambda 44959,5007$ doublet. In many works, disc and outflow are separated according to the width of the two Gaussian components used in fitting the lines (narrower and broader, respectively). In our analysis, this approach is not feasible since disc and outflow components are both present in many spaxels. Furthermore, in the central regions the line profiles are very complex, and their fit requires three or four Gaussians. This means that the distinction between disc and outflow through the width of the fitted components only is not trivial. We note that our approach is not a rigorous method for discriminating between the gas in the outflow and the disc, but a convenient way to analyse in detail the outflow features, as we show in the following.

Because the $\mathrm{H} \alpha$ emission is in general dominated by the disc, while the outflow is enhanced in [O III] (e.g. Venturi et al. 2018), in Fig. 2 we show the $\mathrm{H} \alpha$ disc component flux maps, superimposing the $[\mathrm{O}$ III $] \lambda 5007$ outflow component flux contours (not corrected for dust reddening) for all the galaxies, using the method described above to discriminate between the two components. The blueshifted and redshifted outflowing components are indicated in blue and red, respectively. For each velocity bin, we 


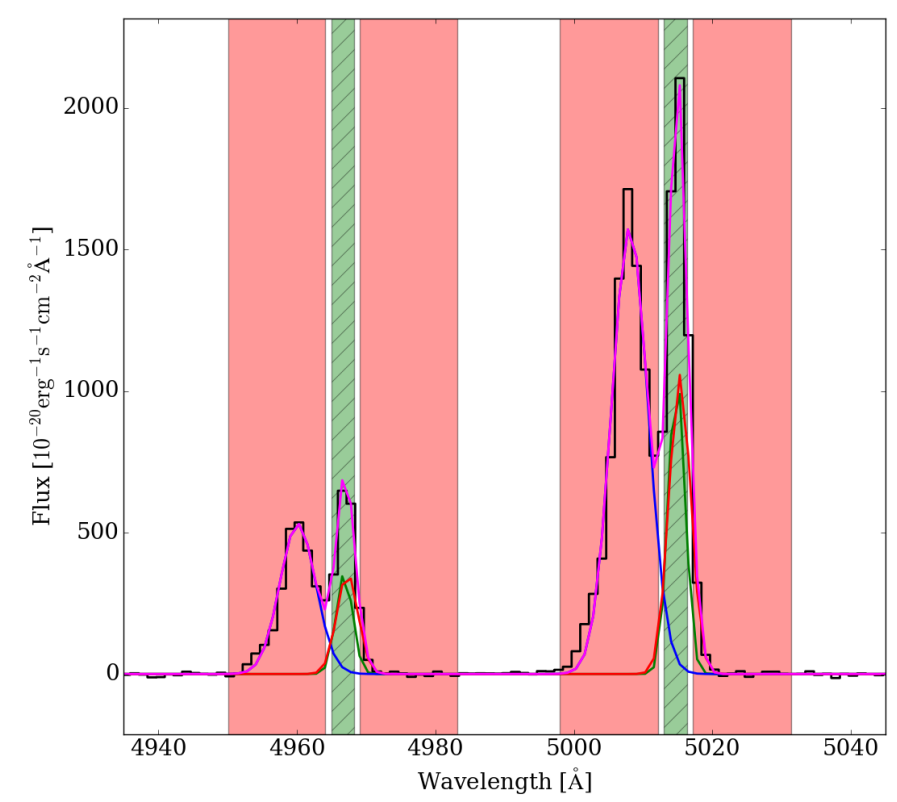

Fig. 1. [O III] $\lambda \lambda 44959,5007$ doublet in a single spaxel spectrum of the continuum-subtracted cube of the Circinus galaxy. The disc $\left(-100 \mathrm{~km} \mathrm{~s}^{-1}<v<100 \mathrm{~km} \mathrm{~s}^{-1}\right)$ and outflow $\left(-1000 \mathrm{~km} \mathrm{~s}^{-1}<v<\right.$ $-150 \mathrm{~km} \mathrm{~s}^{-1}$ and $150 \mathrm{~km} \mathrm{~s}^{-1}<v<1000 \mathrm{~km} \mathrm{~s}^{-1}$ ) components taken into account, highlighted in green with stripes and red, respectively, are superimposed to show an example of our procedure. The disc component is centred to the value of the stellar velocity in this spaxel $\left(\sim 460 \mathrm{~km} \mathrm{~s}^{-1}\right)$, obtained previously by fitting the original data cube with pPXF. The magenta line represents the result of the three-component fit (blue, green, and red Gaussians), performed with MPFIT to reproduce the observed line profiles.

only select the spaxels with a $S / N>5$, computed by dividing the integrated flux in the velocity channels by the corresponding noise. The noise is estimated from the standard deviation of the data-model residuals of the fit around each line (within a range about $60-110 \AA$ wide, depending on the line). Looking at Fig. 2, it can be seen that the disc flux maps and the outflow contours are clearly different from each other. The outflow component is often extended in a kpc-scale conical or biconical distribution, as can be clearly seen in Circinus (north-west cone), IC 5063 (north-west and south-east cones), NGC 2992 (north-west and south-east cones), NGC 4945 (north-west and south-east cones), and NGC 5643 (east and west cones). In Centaurus A the outflow component is mainly distributed in two cones (direction north-east and south-west) in the same direction of the extended double-sided jet revealed both in the radio and X-rays (e.g. Hardcastle et al. 2003), and located perpendicularly with respect to the gas in the disc component. Unfortunately, since for this galaxy we cannot take the stellar velocity as a reference, in some regions we underestimate the gas velocity of the disc component. Consequently, a portion of the disc is still present in the outflowing component. Also in NGC 1365, the outflow component flux map has a biconical shape extended in the south-east and north-west directions, while the disc component appears to be completely different, being dominated by an elongated circumnuclear SF ring and by the bar. Unfortunately, similar to Centaurus A, a portion of the disc is still present in our outflowing gas selection, because of the high velocity reached by the gas along the bar (see Venturi et al. 2018 for more details). NGC 1068 is almost face-on, allowing us to admire the spiral arms of the disc, traced by the disc component, and preventing the outflowing component from having a clear biconical distribution, but to be broadly extended in all the inner region of the observed FOV. As reported in Sect. 2.1, both the ionised and molecular outflow already observed in this galaxy are observed in the north-east and south-west directions (e.g. Cecil et al. 2002; García-Burillo et al. 2014). Finally, in NGC 1386 the outflow component does not show a well-defined conical distribution, but appears to be located in the very inner region of the galaxy, with two elongated structures to the north and south of the nucleus, corresponding to nuclear bipolar outflows already revealed by Ferruit et al. (2000) and Lena et al. (2015).

We note that in almost all galaxies the (bi)conical outflow is detected in both its blueshifted and redshifted components, which are often overlapping. For example, this can be clearly seen in NGC 4945, where the north-west cone has approaching velocities at its edges and receding ones around its axis, while the south-east cone has the opposite behaviour, with receding velocities at its edges and approaching ones around its axis. This was already revealed by Venturi et al. (2017) through the kinematical maps of the $\left[\mathrm{N}_{\text {II }}\right]$ line emission.

\subsection{Extinction, density, and ionisation parameter}

We calculated the visual extinction $A_{V}$ through the Balmer decrement $\mathrm{H} \alpha / \mathrm{H} \beta$, assuming a Calzetti et al. (2000) attenuation law, with $R_{V}=3.1$ (i.e. galactic diffuse ISM) and a fixed temperature of $T_{\mathrm{e}}=10^{4} \mathrm{~K}$. The top panel of Fig. 3 illustrates the extinction map of Circinus obtained for the total line profile (without separating disc and outflow component), where we exclude all the spaxels that have $S / N<5$ on the emission lines involved. In this case, we defined the $\mathrm{S} / \mathrm{N}$ of a line as the ratio between the peak value of the fitted line profile and the standard deviation of the data-model residuals of the fit around the line. The highest values $A_{V} \gtrsim 5$ come from the galaxy disc, while the conical outflow is characterised by $A_{V} \gtrsim 2$. The extinction maps for the other galaxies are shown in Fig. A.1, showing that the highest values of $A_{V}$ come mainly from dust lanes (e.g. Centaurus A and NGC 2992), gas discs as shown for Circinus (e.g. NGC 4945), spiral arms (e.g. NGC 1068 and NGC 1386), or gas flowing along the bar (e.g. NGC 1365).

In order to analyse in detail these differences, we measured two values of $A_{V}$ in each spaxel for every galaxy, one for the disc and one for the outflow component. To do so, for each velocity bin we selected only the spaxels with a $S / N>5$, and we discarded all the velocity bins where $\mathrm{H} \alpha / \mathrm{H} \beta<2.86$ (the theoretical value associated with $A_{V}=0$ ). The top panel of Fig. 4 shows the flux spatial distributions of $A_{V}$ for the disc (in green with stripes) and outflow (in red) components of all the MAGNUM galaxies, with the median of the two distributions superimposed (dotted line). Interestingly, the two distributions are shifted in $A_{V}$ relative to each other, with higher values of extinction in the disc (median: $A_{V} \sim 1.75-\mathrm{H} \alpha / \mathrm{H} \beta \sim 5.51$ ) and lower in the outflow (median: $A_{V} \sim 1.02-\mathrm{H} \alpha / \mathrm{H} \beta \sim 4.16$ ). Therefore, the majority of the outflowing gas appears to be less affected by dust extinction than the disc. We have verified that there is no large distinction between the redshifted and blueshifted outflowing gas distributions due to projection effects, probably due to the fact that redshifted and blueshifted velocities coexist in each cone of the outflow because of geometrical effects (Venturi et al., in prep.).

In order to estimate the gas density, we make use of the optical [S II] $] 6717 /\left[\mathrm{S}_{\text {II }}\right] \lambda 6731$ ratio, converting it to an electron density, using the Osterbrock \& Ferland (2006) model, assuming a temperature of $T_{\mathrm{e}}=10^{4} \mathrm{~K}$. This tracer is density-sensitive in the range $50 \mathrm{~cm}^{-3} \lesssim n_{\mathrm{e}} \lesssim 5000 \mathrm{~cm}^{-3}$, which falls within the 

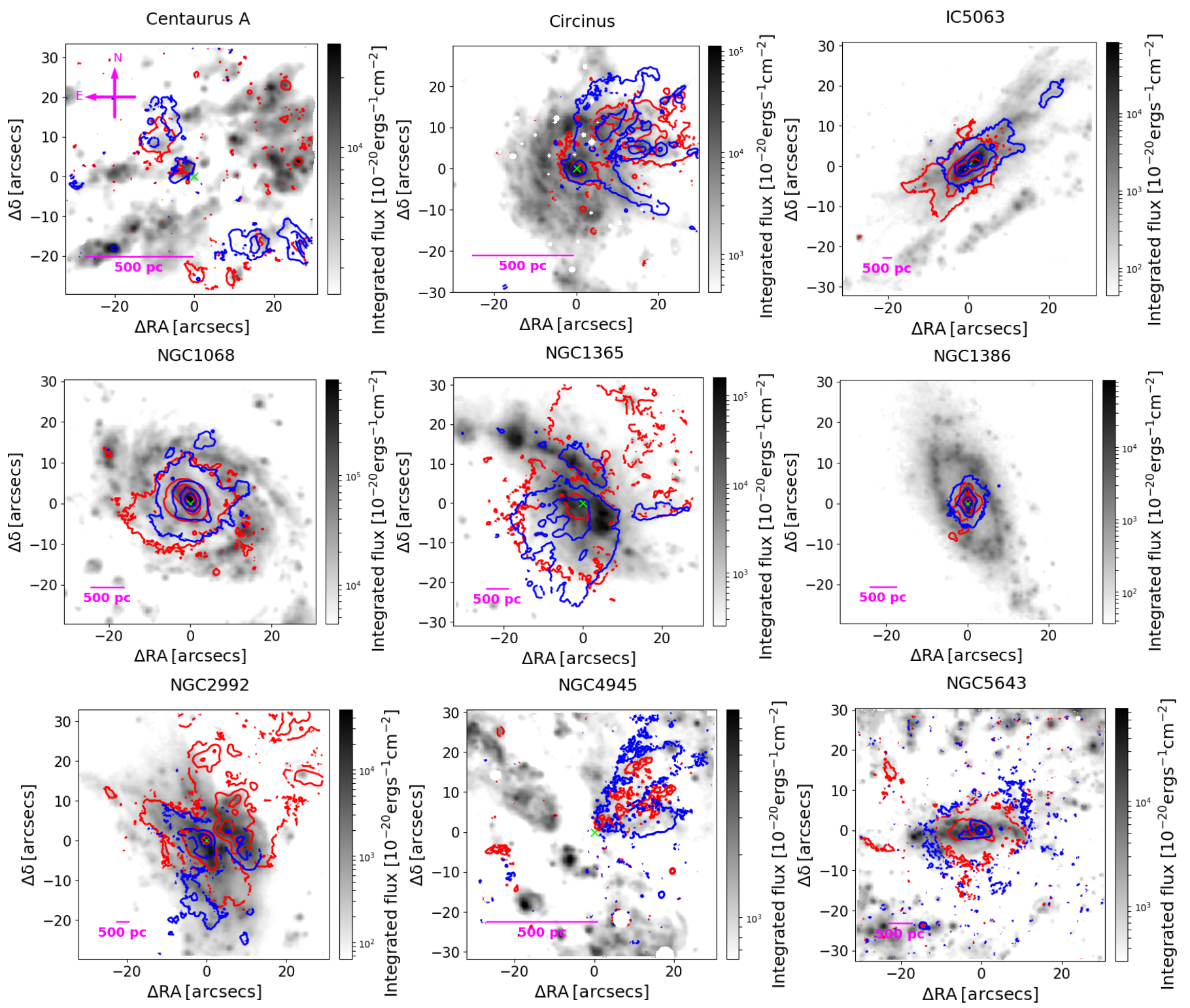

Fig. 2. $\mathrm{H} \alpha$ disc component flux maps (not corrected for dust reddening) for all the galaxies, namely Centaurus A, Circinus, IC 5063, NGC 1068, NGC 1365, NGC 1386, NGC 2992, NGC 4945, and NGC 5643. [O III] 25007 outflow component flux contours are superimposed for all the galaxies. The blueshifted and redshifted outflow emission (in blue and red, respectively) is often extended in a kiloparsec-scale conical or biconical distribution. For each velocity bin, we show only the spaxels with a $S / N>5$. The magenta bar represents a physical scale of $\sim 500 \mathrm{pc}$. East is to the left, as shown in the first image on the left. The white circular regions are masked foreground stars. The cross marks the position either of the Type 1 nucleus (i.e. peak of the broad $\mathrm{H} \alpha$ emission), if present, or the peak of the continuum in the wavelength range $6800-7000 \AA$.

typically estimated range of densities in the extended NLR (e.g. Perna et al. 2017). Below and above these densities, the flux ratio of the doublet saturates. The central panel of Fig. 3 reports the density map of Circinus, obtained for the total line profile, that appears clearly non-uniform, as found by Kakkad et al. (2018), with enhanced values of density up to $n_{\mathrm{e}} \sim 10^{3} \mathrm{~cm}^{-3}$ located in the inner regions of the galaxy and along the northwestern outflowing cone. The density maps of the other galaxies in the sample are reported in Fig. B.1. The majority of them shows the same characteristics found in Circinus. The highest densities, with peaks of up to $n_{\mathrm{e}} \sim 10^{3} \mathrm{~cm}^{-3}$ (e.g. IC 5063, NGC 1068, NGC 1386), are mainly found in the central regions of the galaxies. High densities $\left(10^{2} \mathrm{~cm}^{-3}<n_{\mathrm{e}}<10^{3} \mathrm{~cm}^{-3}\right)$ are also found along the outflowing cone axis, as is clearly visible in the north-east and south-west directions in NGC 1068 and in the double-side cone of NGC 4945 (north-west-south-east direction). On the contrary, NGC 1365 has enhanced values of density around the circumnuclear star-forming ring (see also Kakkad et al. 2018; Venturi et al. 2018), consistent with recent results showing a correlation between density and the location of the SF regions (e.g. Westmoquette et al. 2011, 2013; McLeod et al. 2015). The central panel of Fig. 4 shows the density distribution of all the galaxies for the disc (in green with stripes) and outflow (in red) components, separately. The two distributions peak at the minimum value of the electron density range taken into account (i.e. $n_{\mathrm{e}}=50 \mathrm{~cm}^{-3}$ ), but the disc median value is $n_{\mathrm{e}} \sim 130 \mathrm{~cm}^{-3}$ against $n_{\mathrm{e}} \sim 250 \mathrm{~cm}^{-3}$ for the outflowing gas, and thus the outflow is generally denser than the disc gas in MAGNUM galaxies.

Previous works (e.g. Holt et al. 2011; Arribas et al. 2014; Villar Martín et al. 2014, 2015) have found very high reddening and densities associated with ionised outflows in local objects 


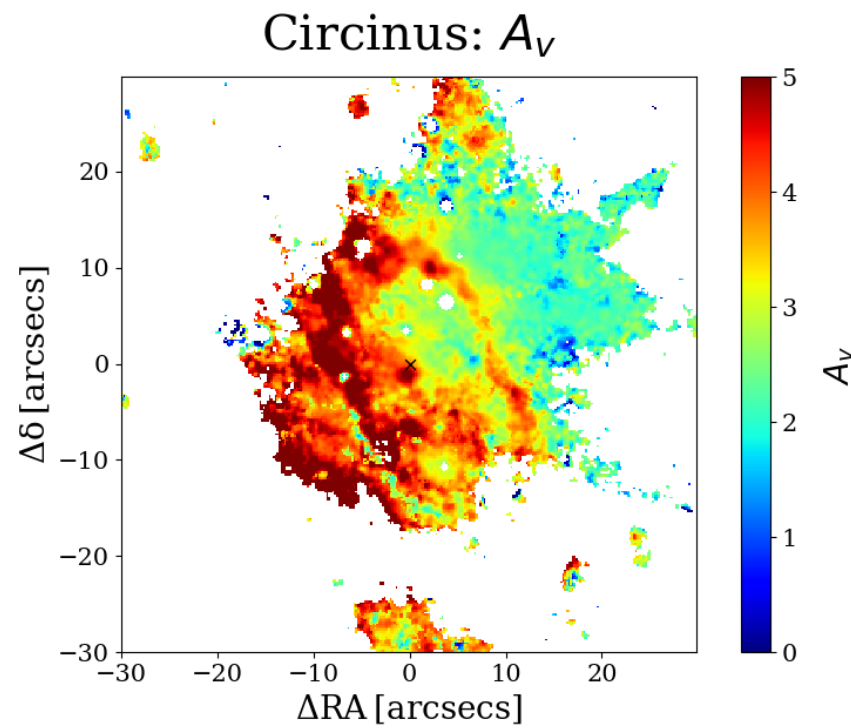

(e.g. $\mathrm{H} \alpha / \mathrm{H} \beta \sim 4.91$ and $n_{\mathrm{e}} \gtrsim 1000 \mathrm{~cm}^{-3}$, Villar Martín et al. 2014). Concerning the reddening, although we find that the outflowing gas is generally less affected by dust extinction than the disc, the median value of the total distribution is significantly affected by dust $(\mathrm{H} \alpha / \mathrm{H} \beta \sim 4.16)$, with tails up to $\mathrm{H} \alpha / \mathrm{H} \beta \gtrsim 6$. Similarly, the outflow density of MAGNUM galaxies is higher than the values in the disc gas, but appears to be far lower than the values found by these authors. This could stem from the fact that the galaxies studied by Holt et al. (2011), Arribas et al. (2014) are local luminous or ultra-luminous infrared galaxies (U/LIRGs), and those of Villar Martín et al. (2014, 2015) are highly obscured Seyfert 2, thus sampling sources that are more gas and dust rich compared to our sample. However, our values are also lower than the outflow densities found in Perna et al. (2017) $\left(n_{\mathrm{e}} \sim 1200 \mathrm{~cm}^{-3}\right)$, who targeted optically selected AGNs from the SDSS, and Förster Schreiber et al. (2018b), who presented a census of ionised gas outflows in high- $z$ AGN with the $\mathrm{KMOS}^{3 \mathrm{D}}$ survey $\left(n_{\mathrm{e}} \sim 1000 \mathrm{~cm}^{-3}\right)$. A possible explanation could be related to the high quality of our MUSE data, which also allows us to detect the faint $[\mathrm{S}$ II] emission associated with lower density regions. If we calculate the median densities of the disc and outflow components, weighting for the [S II] line flux, we obtain higher values $\left(n_{\mathrm{e}} \sim 170 \mathrm{~cm}^{-3}\right.$ and $n_{\mathrm{e}} \sim 815 \mathrm{~cm}^{-3}$, for disc and outflow, respectively). This shows that previous outflow density values from the literature could be biased towards higher $n_{\mathrm{e}}$ because they are based only on the most luminous outflowing regions, characterised by a higher $\mathrm{S} / \mathrm{N}$. This could also mean that outflows at high- $z$ could be far more extended than the values we observe.

Finally, we traced the ionisation parameter $U$, defined as the number of ionising photons $S_{*}$ per hydrogen atom density $n_{\mathrm{H}}$, divided by the speed of light $c$, making use of the $\left[\mathrm{S}_{\text {III }}\right] \lambda \lambda 9069,9532 /[\mathrm{S}$ II $] \lambda \lambda 6717,31$ ratio $^{1}$ (e.g. Díaz et al. 2000). Since $\left[S_{\text {III }}\right] \lambda 9532$ is not covered by the wavelength range observed by MUSE, we adopted a theoretical ratio of $\left[\mathrm{S}_{\mathrm{III}}\right] \lambda \lambda 9532 /[\mathrm{S}$ III $] \lambda \lambda 9069=2.5$ (Vilchez \& Esteban 1996), fixed by atomic physics. The parameter $U$ represents a measure of the intensity of the radiation field, relative to gas density. It can be traced using the ratios of emission lines of different ionisation stages of the same element: the larger the difference in ionisation potentials of the two stages, the better the ratio will constrain $U$. The [S $\left.\mathrm{S}_{\text {III }}\right] \lambda \lambda 9069,9532 /\left[\mathrm{S}_{\mathrm{II}}\right] \lambda \lambda 6717,31$ ratio is a more reliable diagnostic than [O III] $\lambda \lambda 4959,5007 /\left[\mathrm{O}_{\text {II }}\right] \lambda \lambda 3726,28$, since it is largely independent on metallicity (Kewley \& Dopita 2002).

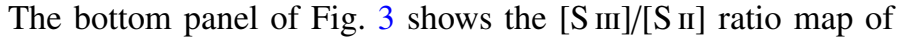
Circinus where $\left[\mathrm{S}_{\mathrm{III}}\right] /\left[\mathrm{S}_{\mathrm{II}}\right]$ reaches values as high as $\sim 2$ along the conical outflow. Figure C. 1 reports the $\left[\mathrm{S}_{\mathrm{III}}\right] /\left[\mathrm{S}_{\mathrm{II}}\right]$ maps for the other galaxies. Similar to Circinus, several other galaxies in our sample (NGC 1068, IC 5063, NGC 1386, NGC 2992, and NGC 5643) also show higher values of [S III]/[S II $]$ along the outflow axis. In addition, $\left[\mathrm{S}_{\mathrm{III}}\right] /\left[\mathrm{S}_{\mathrm{II}}\right]$ is high in the starforming region embedded in the gas disc in Centaurus $\mathrm{A}$, and located in isolated blobs in NGC 1365. Unfortunately, the $\left[\mathrm{S}_{\mathrm{III}}\right] /[\mathrm{S}$ II] ratio is poorly constrained in NGC 4945 . The bottom panel of Fig. 4 shows a comparison between the distribution of $\left[\mathrm{S}_{\mathrm{III}}\right] /\left[\mathrm{S}_{\mathrm{II}}\right]$ associated with the disc component (in green with stripes) and with the outflow (in red). The top $x$-axis translates the $\left[\mathrm{S}_{\mathrm{III}}\right] /\left[\mathrm{S}_{\mathrm{II}}\right]$ ratio into $\log (U)$, following the relation provided by Díaz et al. (2000), assuming that the gas is optically thin (Morisset et al. 2016). In conclusion, $\log \left(\left[\mathrm{S}_{\mathrm{III}}\right] /\left[\mathrm{S}_{\mathrm{II}}\right]\right)$ is observed in the range from $\sim-1$ to $\sim 1$, with higher values associated with the outflowing gas (median:

Fig. 3. ISM properties in the Circinus galaxy. Top panel: map of the total extinction in $V$ band $A_{V}$ obtained from the Balmer decrement $\mathrm{H} \alpha / \mathrm{H} \beta$. Only spaxels with $\mathrm{H} \alpha$ and $\mathrm{H} \beta S / N>5$ are shown. Central panel: map of the total electron density measured from the $\left[\mathrm{S}_{\mathrm{II}}\right] \lambda 6717 /\left[\mathrm{S}_{\mathrm{II}}\right] \lambda 6731$ ratio. Only spaxels with $\left[\mathrm{S}_{\mathrm{II}}\right] \lambda 6717$ and [S II] $] 6731 S / N>5$ are shown. Bottom panel: map of the $\left[\mathrm{S}_{\text {III }}\right] \lambda \lambda 9069,9532 /\left[\mathrm{S}_{\text {II }}\right] \lambda \lambda 6717,31$ ratio, proxy for the ionisation parameter. Only spaxels with [S III] $\lambda \lambda 9069,9532$ and [S II] $\lambda \lambda 6717,31$ $S / N>5$ are shown.

1 Both emission lines were corrected for dust attenuation prior to deriving the ionisation parameter. 

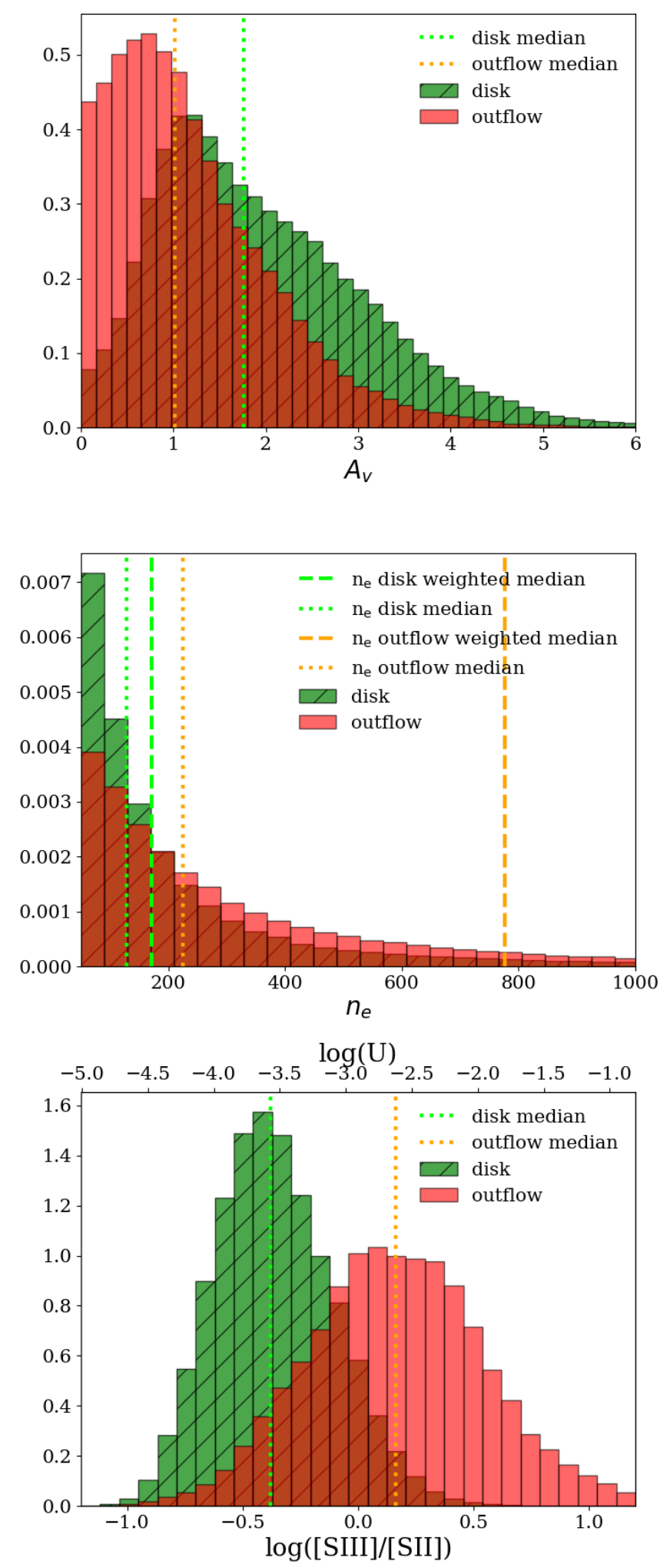

Fig. 4. Comparison of the disc (green with stripes) and outflow (red) component distributions for all the MAGNUM galaxies in terms of visual extinction $A_{V}$, density $n_{\mathrm{e}}$, and $\log \left(\left[\mathrm{S}_{\mathrm{III}}\right] /\left[\mathrm{S}_{\mathrm{II}}\right]\right.$ ) (top, middle, and bottom panel, respectively). The two distributions are normalised such that the integral over the range is 1 . For each velocity bin, we take into account only the spaxels with a $S / N>5$ for the emission lines involved. The light green and orange dotted lines represent the median of the disc and outflow distributions, respectively. The dashed lines in the central panel represent the median value weighted on the $\left[\mathrm{S}_{\mathrm{II}}\right]$ line flux. The top $x$-axis of the bottom panel translates the $\left[\mathrm{S}_{\mathrm{III}}\right] /[\mathrm{S}$ II] ratio into $\log (U)$, following the relation provided by Díaz et al. (2000). $\left.\log \left(\left[\mathrm{S}_{\mathrm{III}}\right] /\left[\mathrm{S}_{\mathrm{II}}\right]\right) \sim 0.16, \log (U) \sim-2.75\right)$ with respect to the disc (median: $\log \left([\mathrm{S}\right.$ III $\left.] /\left[\mathrm{S}_{\mathrm{II}}\right]\right) \sim-0.38, \log (U) \sim-3.60$ ), where it traces mainly star-forming regions. The median value found for the disc component is slightly lower than the ionisation parameters found in nearby star-forming galaxies in other studies (e.g. Dopita et al. 2006; Liang et al. 2006; Kewley \& Ellison 2008; Nakajima \& Ouchi 2014; Bian et al. 2016), obtained through the [O III $] \lambda \lambda 4959,5007 /\left[\mathrm{O}_{\text {II }}\right] \lambda \lambda 3726,28$. We also calculated the median values of the extinction and ionisation parameter distributions, weighting for the line fluxes of the involved lines, finding negligible $\left(\Delta A_{V} \sim-0.02, \Delta \log ([\mathrm{S}\right.$ III $] /[\mathrm{S}$ II $\left.]) \sim+0.03\right)$ and small differences $\left(\Delta A_{V} \sim-0.20, \Delta \log \left(\left[\mathrm{S}_{\mathrm{III}}\right] /\left[\mathrm{S}_{\mathrm{II}}\right]\right) \sim+0.25\right)$ with respect to the median values shown in Fig. 4, for the disc and outflow component, respectively.

In summary, we find that the disc and outflow are characterised by different properties. In the next section, we investigate the ionisation properties of the gas separately for the two kinematic components.

\subsection{Spatially and kinematically resolved BPT}

Spatially and kinematically resolved BPT diagrams allow us to explore the dominant contribution to ionisation in each spaxel in the disc and outflow components separately (a similar approach has been used by e.g. Westmoquette et al. 2012; McElroy et al. 2015; Karouzos et al. 2016b).

The left and middle panels of Fig. 5 show the [N II]- and [S II]-BPT diagrams of Circinus for the disc and outflow components, respectively. For each velocity bin, we select only the spaxels with a $S / N>5$, computed by dividing the integrated flux in the velocity channels for each emission line by the corresponding noise. The dashed curve is the boundary between star-forming galaxies and AGN defined by Kauffmann et al. (2003), while the solid curve is the theoretical upper limit on SF line ratios found by Kewley et al. (2001). The dotted line, instead, is the boundary between Seyferts and LI(N)ERs introduced by Kewley et al. (2006). The dominant source of ionisation is colour-coded: shades of blue for SF, green for intermediate regions in the [N $\mathrm{NI}_{\mathrm{II}}$-BPT and $\mathrm{LI}(\mathrm{N}) \mathrm{ER}$ in the [S $\left.\mathrm{S}_{\mathrm{II}}\right]-\mathrm{BPT}$, and red for AGN-like ionising spectra, as a function of the $x$ axis line ratios (darker shades means higher $x$-axis line ratios). The LI(N)ER-like excitation can be due either to shock excitation (e.g. Dopita \& Sutherland 1995) or to hard- $X$ radiation coming from AGN and to hot evolved (post-asymptotic giant branch) stars (e.g. Singh et al. 2013; Belfiore et al. 2016). The corresponding position on the map of the outflowing gas component, colour-coloured based on the different sources of ionisation, is shown in the right panels of Fig. 5. In the background of all the pictures (black dots in the BPTs and shaded grey in the corresponding maps), we show the disc and outflow components together to allow a better visual comparison.

Looking at Fig. 5, we note that the outflow spans a wider range of $[\mathrm{N} \mathrm{II}] / \mathrm{H} \alpha$ and $\left[\mathrm{S}_{\mathrm{II}}\right] / \mathrm{H} \alpha$, including lower and higher values compared to the disc. Specifically, the highest and lowest values of low-ionisation line ratios (LILrs; i.e. [N $\left.\mathrm{II}_{3}\right] \mathrm{H} \alpha$ and $[\mathrm{S}$ II $] / \mathrm{H} \alpha$ ), displayed in dark red and orange, are prominent in the AGN/LI(N)ER-dominated outflow component, while they are not observable in the disc component. This can also be seen

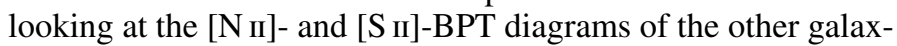
ies, shown in Figs. D.1-D.4, respectively ${ }^{2}$. In the following we

2 [N II]- and [S II]-BPT diagrams in the appendix were made taking into account the smoothed datacubes. An exception is made for NGC 1365 , where the biconical outflow can be well traced only in the Voronoibinned cube. 

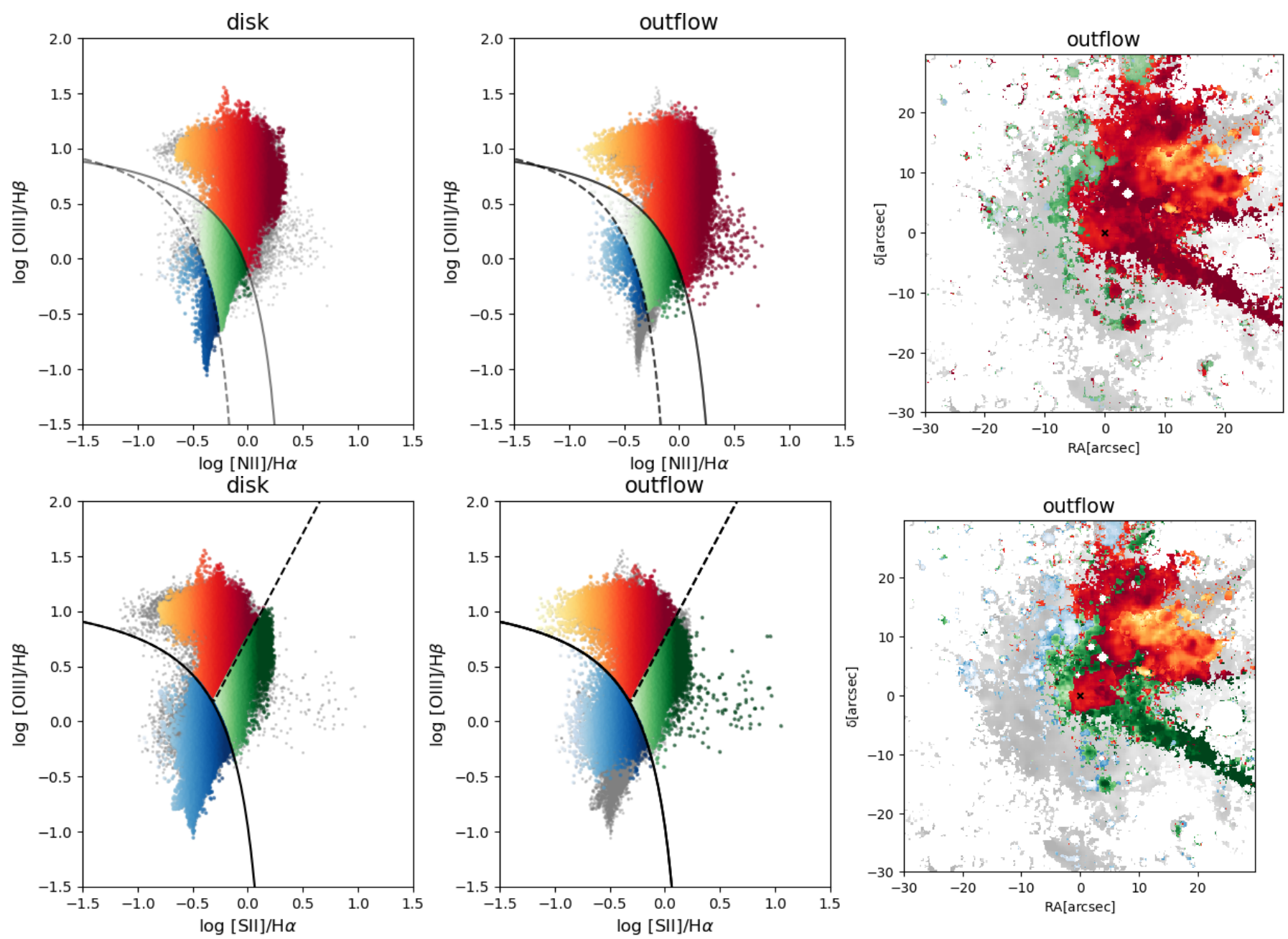

Fig. 5. Left and central panels: [ $\left.\mathrm{N}_{\mathrm{II}}\right]-$ and [ $\left.\mathrm{S}_{\mathrm{II}}\right]-\mathrm{BPT}$ diagrams for the disc and outflow components, on the left and on the right respectively, of Circinus. Blue denotes SF-dominated regions, green intermediate regions in the [N II]-BPT and LI(N)ER in the [S II]-BPT, and red AGN-like ionised spectra, colours-coded as a function of the $x$-axis line ratios (darker shades means higher $x$-axis line ratios). The black dashed curve is the boundary between star-forming galaxies and AGN defined by Kauffmann et al. (2003), while the black solid curve is the theoretical upper limit on SF line ratios found by Kewley et al. (2001). The black dotted line is the Kewley et al. (2006) boundary between Seyferts and LI(N)ERs. Right panels: [N $\left.\mathrm{N}_{\mathrm{II}}\right]-$ and [ $\left.\mathrm{S}_{\mathrm{II}}\right]$-BPT maps of the outflowing gas component, colour-coded according to BPTs. In the background of all the pictures (grey dots in the BPTs and shaded grey in the corresponding maps), we show the disc and outflow component together. For each velocity bin, we select only the spaxels with a $S / N>5$ for all the lines involved.

analyse the regions showing the lowest (Sect. 3.2.1) and the highest (Sect. 3.2.2) LILrs.

\subsubsection{Outflowing gas: the lowest $[\mathrm{N} \Perp] / \mathrm{H} \alpha-[\mathrm{S} \Perp] / \mathrm{H} \alpha$ line ratios}

As Fig. 5 shows, the lowest $\left[\mathrm{N}_{\mathrm{II}}\right] / \mathrm{H} \alpha$ and $\left[\mathrm{S}_{\mathrm{II}}\right] / \mathrm{H} \alpha$ line ratios (in orange, with $\log ([\mathrm{N}$ II $] / \mathrm{H} \alpha) \lesssim-0.5$ and $\log ([\mathrm{S}$ II $] / \mathrm{H} \alpha) \lesssim-0.8)$ in the AGN ionisation regime belong almost exclusively to the outflowing gas. Looking at the right panel of Fig. 5, it can be seen that these values correspond to the innermost parts of the northwestern cone (in orange) $)^{3}$.

These low values of $\left[\mathrm{N}\right.$ II] $/ \mathrm{H} \alpha$ and $\left[\mathrm{S}_{\mathrm{II}}\right] / \mathrm{H} \alpha$ (with logarithmic values down to $\sim-1$ ) in the AGN-dominated region are not restricted to the Circinus outflowing gas, but appear to be typical outflow signatures in almost all the galaxies of our

\footnotetext{
3 Unfortunately, part of the outflowing gas in the west cone of Circinus is oriented along the plane of the sky, and thus has low line-of-sight velocity. Consequently, the disc component is partly contaminated by the outflow, explaining the presence of the orange dots in the left panels of the BPT diagrams in Fig. 5.
}

sample (NGC 2992, NGC 1365, IC 5063, NGC 1068), and, albeit less clearly, in Centaurus A, NGC 1386, and NGC 5643 (see left panels of Figs. D.1 and D.2, for [N $\mathrm{N}_{\mathrm{II}}$-BPT diagrams, Figs. D.3 and D.4, for [S II]-BPT diagrams). Specifically, the lowest LILrs dominate the majority of the visible north-west cone of NGC 2992, which extends out to $\sim 5 \mathrm{kpc}$, and the innermost parts of the cones in most of the other galaxies (northwest cone in NGC 1365, north-west and south-east directions in IC 5063, north-east and south-west directions in NGC 1068, east and west directions in NGC 5643), highlighted in orange in the right panels of Figs. D.1-D.4, for the [N II]- and the [S II]-BPT diagrams, respectively. On the contrary, these features seem to be completely absent in NGC 4945 .

\subsubsection{Outflowing gas: the highest $[\mathrm{N} \Perp] / \mathrm{H} \alpha$ and $[\mathrm{S} \Perp] / \mathrm{H} \alpha$ line ratios}

Looking at Fig. 5, the highest $\left[\mathrm{N}_{\mathrm{II}}\right] / \mathrm{H} \alpha$ and $\left[\mathrm{S}_{\mathrm{II}}\right] / \mathrm{H} \alpha$ line ratios (in darker red and green, respectively), with values higher than 0 , appear to come from the edges of the outflowing cone. 
As noted above for the other ratios, this feature is not only typical of Circinus, but appears to characterise almost all of the sample (NGC 4945, NGC 2992, NGC 1068, Centaurus A, IC 5063, NGC 1386 and NGC 5643), as can be seen in the left panels of Figs. D.1-D.4, for [N II]- and [S II]-BPT diagrams, respectively. For instance, it is clearly visible in NGC 4945, where - as in Circinus - the high LILrs come from the external edges of both the north-western and the south-eastern outflowing cones, even though in the latter only the edges are barely observable because of the low $\mathrm{H} \beta \mathrm{S} / \mathrm{N}$. In NGC 1068, IC 5063 and in NGC 5643, this enhancement is extended from the edges of the cones to a region almost perpendicular to the outflow axis ${ }^{4}$ where a conical dark red region (AGN-dominated) in [N $\left.\mathrm{N}_{\text {II }}\right]-\mathrm{BPT}$ and a conical dark green region (LI(N)ER-dominated) in [S II]BPT can be distinguished.

On the contrary, it can be seen that in NGC 1365 the highest LILrs stem from gas located in the disc component in the vicinity of the outflowing cone edges. However, we believe that the distinction between disc and outflow components is not completely defined in this galaxy. Part of the outflowing gas could have an apparent low line-of-sight velocity because it is oriented along the plane of sky, contaminating the disc component.

\section{Discussion}

In the analysis of the kinematically and spatially resolved BPT diagrams for our sample of galaxies (see Sect. 3.2), we identify two main features in the outflowing gas: a decrease and an enhancement in the $[\mathrm{N}$ II $] / \mathrm{H} \alpha$ and $\left[\mathrm{S}_{\mathrm{II}}\right] / \mathrm{H} \alpha$ line ratios, coming from distinct regions of the outflowing cones. In the following, we discuss possible explanations for these features, such as a combination of optically thin and optically thick clouds or shocks and/or a hard filtered radiation field from the AGN. To do this, we compare ionisation state and gas velocity dispersion with [N $\mathrm{II}]$ - and [S II]-BPT diagrams comprising the whole MAGNUM sample; the only exception is NGC 1068, which shows a different behaviour with respect to the others concerning

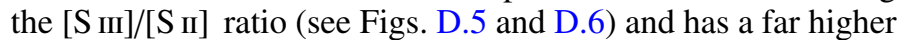
velocity dispersion (see Figs. D.7 and D.8). Therefore, it will be discussed in a forthcoming paper.

\subsection{Possible scenarios for the lowest LILrs}

Figure 6 shows the $\left[\mathrm{N}_{\mathrm{II}}\right]-$ and $\left[\mathrm{S}_{\mathrm{II}}\right]-\mathrm{BPT}$ diagrams of all the MAGNUM galaxies (excluding NGC 1068), colour-coded as a function of the $\left[\mathrm{S}_{\mathrm{III}}\right] \lambda \lambda 9069,9532 /\left[\mathrm{S}_{\mathrm{II}}\right] \lambda \lambda 6717,31$ ratio, which is a proxy for the ionisation parameter, as reported in the corresponding colour bars (darker shades means higher ionisation). Here, we use the Voronoi-binned cubes to increase the S/N and, for each Voronoi bin, we select only the velocity bins where the $\left[\mathrm{S}_{\text {III }}\right.$ and $\left[\mathrm{S}_{\text {II }}\right]$ line fluxes have a $S / N>3$. The lowest LILrs $\left(\log \left(\left[\mathrm{N}_{\mathrm{II}}\right] / \mathrm{H} \alpha\right) \sim-1\right.$ and $\left.\log \left(\left[\mathrm{S}_{\mathrm{II}}\right] / \mathrm{H} \alpha\right) \sim-1\right)$, which mainly trace the innermost regions of the outflowing gas, also correspond to the highest $\left[\mathrm{S}_{\mathrm{III}}\right] /\left[\mathrm{S}_{\mathrm{II}}\right]$ line ratio ([S III $] /\left[\mathrm{S} \mathrm{III}_{2} \sim 2\right.$ ), which decreases to values $[\mathrm{S}$ III $] /[\mathrm{S}$ II] $\lesssim 0.5$ going towards higher LILr values.

This observed variation in the degree of excitation can be interpreted as being due to diverse proportions of two populations of line emitting clouds, characterised by different levels of opacity to the radiation, a matter-bounded (MB)

\footnotetext{
4 In NGC 1068, IC 5063 and NGC 5643 the outflow axis is in the direction north-east and south-west, north-west and south-east, and east and west, respectively.
}

and an ionisation-bounded (IB) component, first discussed by Binette et al. (1996). The former is only responsible for the highionisation lines (e.g. [O III] $\lambda \lambda 44959,5007)$, while the latter is also characterised by low-ionisation ones (e.g. [ $\left.\mathrm{N}_{\mathrm{II}}\right] \lambda \lambda 6548,84$ and [S $\left.\left.\mathrm{SI}_{\mathrm{II}}\right] \lambda 6717,31\right)$. Specifically, a cloud is said to be MB if the outer limit to the $\mathrm{H}^{+}$region is marked by the outer edge of the cloud, which means that it is ionised throughout and is optically thin to the incident radiation field. On the other hand, the cloud is IB (or radiation bounded) if the outer limit to the $\mathrm{H}^{+}$region is defined by a hydrogen ionisation front, so both warm ionised and cold neutral regions coexist, and thus the $\mathrm{H}^{+}$region is optically thick to the hydrogen-ionising radiation, absorbing nearly all of it. The original aspect of the Binette et al. (1996) approach is the assumption that the IB clouds are photoionised only by hard photons which have leaked through the MB clouds, meaning that the input ionising spectrum impinging on the IB clouds has been partially filtered (see Fig. 3, Binette et al. 1996). Hard photons interact much less effectively with the gas, being their energy far above the $\mathrm{H}^{0}$ ionisation threshold. This means that, unlike the MB clouds which share the same excitation (i.e. the same ionisation parameter) being coupled with the radiation field, the IB clouds are not necessarily characterised by a unique value of ionisation parameter. Their modelling is parametrised by the ratio of the solid angles subtended by MB and IB clouds $\left(A_{\mathrm{M} / \mathrm{I}}\right)$, which introduces a new free parameter: the geometry of the system, which is often difficult to constrain.

In Fig. 6, the blue dash-dotted line represents the Binette $A_{\mathrm{M} / \mathrm{I}}$ sequence (Binette et al. 1996; Allen et al. 1999), that closely matches the differences in the line ratios that we observe: in the upper left region $\left(A_{\mathrm{M} / \mathrm{I}} \sim 10\right)$, the MB component dominates, meaning that the gas is more optically thin, and is thus characterised by high-ionisation lines, such as [O III] and [S III], tracing the inner gas within the outflowing cones (see Sect. 3.2); going to higher LILrs, the optically thick IB clouds, characterised by a much lower excitation, start playing a major role $\left(A_{\mathrm{M} / \mathrm{I}} \sim 0.001\right)$ both in the outflowing gas and in the disc gas. However, even though the MB-IB dichotomy accounts for the low LILrs, it cannot explain the highest LILrs that we observe in our sample.

Baldwin-Phillips-Terlevich diagrams colour-coded as a function of $\left[\mathrm{S}_{\mathrm{III}}\right] /\left[\mathrm{S}_{\mathrm{II}}\right]$ for each galaxy can be seen in Fig. D.5 and Fig. D.6, for the [N $\left.\mathrm{NI}_{\mathrm{II}}\right]$ - and $\left[\mathrm{S}_{\mathrm{II}}\right]$-BPT diagrams, respectively ${ }^{5}$. The trend found between the $\left[\mathrm{S}_{\text {III }}\right] /\left[\mathrm{S}_{\text {III }}\right]$ line ratio and low LILrs is mainly visible in Circinus, NGC 2992, and NGC 5643, but also in NGC 1386, even though less clearly because of the cut in $\mathrm{S} / \mathrm{N}$. We note that NGC 4945, which is the only galaxy that lacks the MB clouds features, is characterised either by a very strong obscuration of AGN UV radiation or by an AGN that is highly deficient in its UV with respect to X-ray emission (Marconi et al. 2000). This could explain why we see only the IB cloud component, ionised by hard photons filtered by the dust.

In the top panel of Fig. 7, we show the stacked spectrum in the wavelength range 4850-7500 $\AA$ of the region corresponding to the lowest LILrs in Circinus. Specifically, we selected and summed the AGN- and LI(N)ER-dominated spaxels from the original data cube, where $\log ([\mathrm{N}$ II $] / \mathrm{H} \alpha)<-0.2$ and $\log \left(\left[\mathrm{S}_{\mathrm{II}}\right] / \mathrm{H} \alpha\right)<-0.4$, which correspond to the innermost parts of the outflowing cone, indicated in orange in the right panels of

5 The [N II]- and [S II]-BPT diagrams given in the appendix, apart from NGC 1365, are made taking into account the smoothed datacubes, and not the Voronoi-binned ones that we use in this section to compare all the galaxies. 


\section{[NII] BPT diagram}
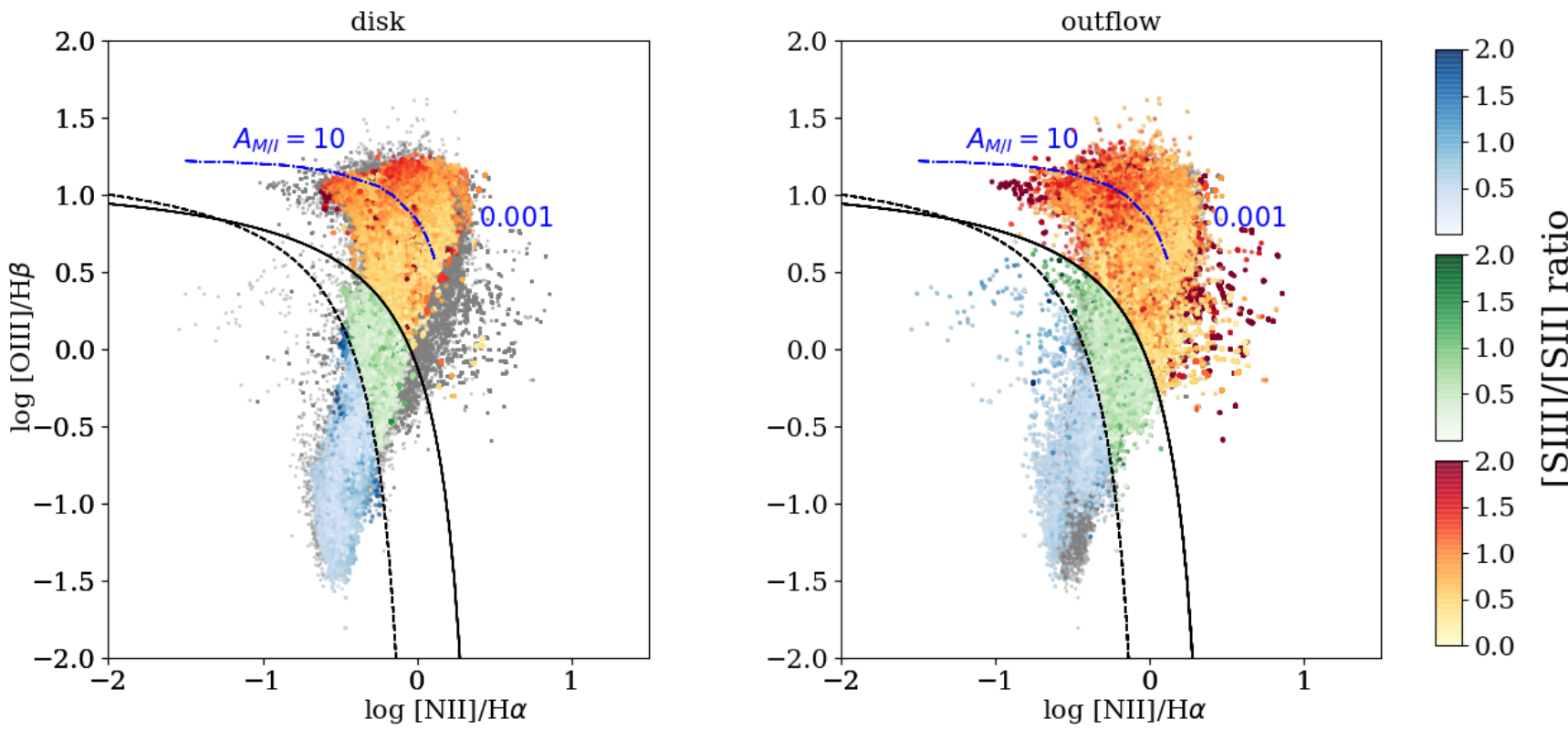

[SII] BPT diagram
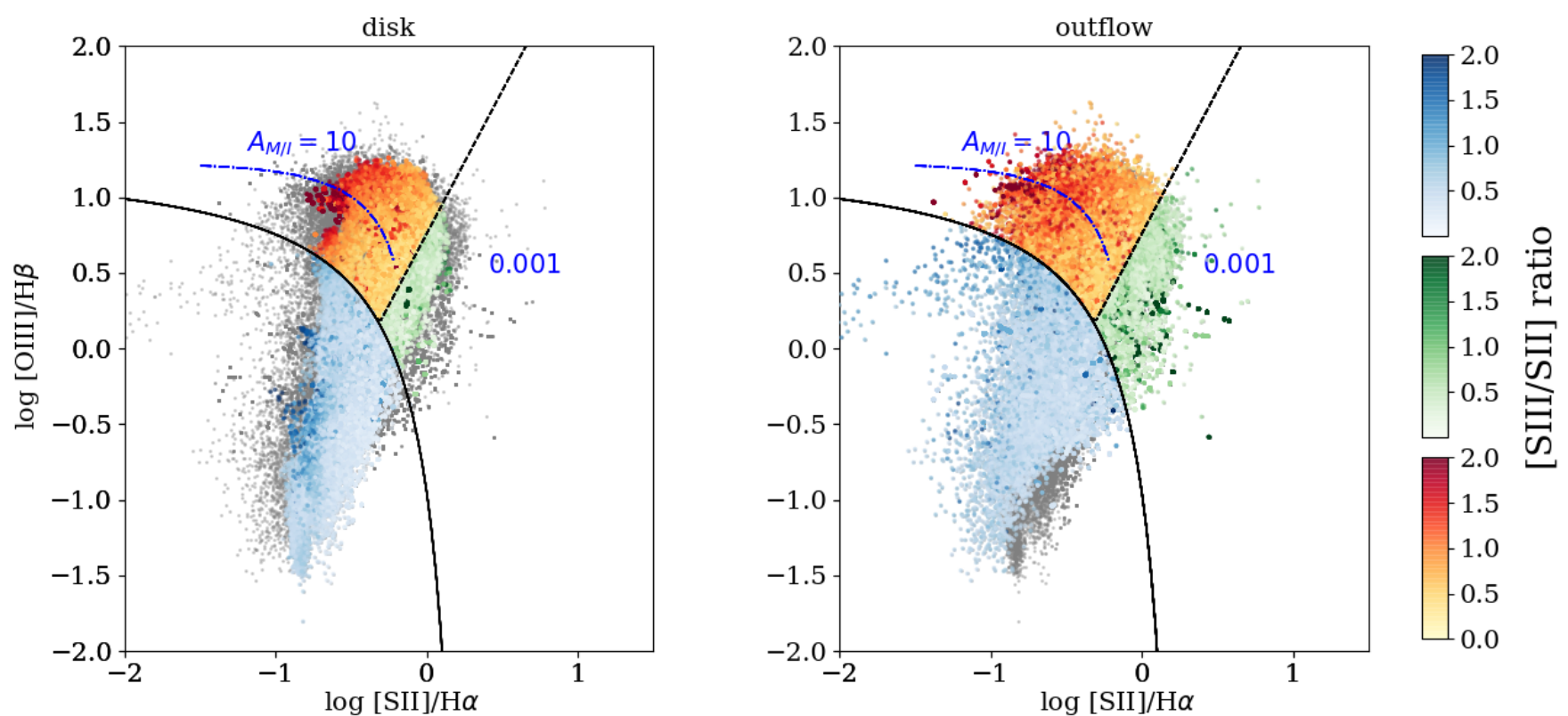

Fig. 6. $\left[\mathrm{N}_{\mathrm{II}}\right]-$ and $\left[\mathrm{S}_{\mathrm{II}}\right]-\mathrm{BPT}$ diagrams for the disc and outflow components, on the left and the right respectively, of all the MAGNUM galaxies, apart from NGC 1068, colour-coded as follows: shades of blue for SF, green for intermediate regions in the [N II]-BPT and LI(N)ER in the [S II]-BPT, and red for AGN-like ionising spectra, as a function of the $\left[\mathrm{S}_{\text {III }}\right] /\left[\mathrm{S}_{\text {III }}\right]$ line ratio (darker shades means higher $\left[\mathrm{S}_{\text {III }}\right] /\left[\mathrm{S}_{\text {III }}\right]$ values). The black dashed curve is the boundary between star-forming galaxies and AGN defined by Kauffmann et al. (2003), while the black solid line is the theoretical upper limit on SF line ratios found by Kewley et al. (2001). The dash-dotted blue curve represents the Binette $A_{\mathrm{M} / \mathrm{I}}$-sequence, dominated by MB clouds in the upper left region and by IB clouds going towards higher LILrs. The black dotted line is the Kewley et al. (2006) boundary between Seyferts and LI(N)ERs. The black dots in the BPTs are all the spaxels taken into account (both of the disc and of the outflow component) with a $S / N>3$ for the line fluxes involved.

Fig. 5. After fitting and subtracting the continuum with pPXF, we fitted the main emission lines with MPFIT, using one Gaussian component with the same velocity and velocity dispersion for each line. We applied the same procedure to obtain the stacked spectrum of the highest LILrs in Circinus $\left(\log \left(\left[\mathrm{N}_{\mathrm{II}}\right] / \mathrm{H} \alpha\right)>0.1\right.$ and $\log ([\mathrm{S}$ II $] / H \alpha)>0)$, shown in the bottom panel of Fig. 7. In Table 1 the observed and dereddened fluxes of the fitted emission lines with respect to the $\mathrm{H} \beta$ are reported for the two spec- tra. It can be seen that the Fe coronal lines (e.g. [Fe vi] $\lambda 5146$, $[\mathrm{Fe}$ xIv $] \lambda 5303,[\mathrm{Fe}$ vII $] \lambda 5721,[\mathrm{Fe}$ vII $] \lambda 6087$, [Fe x] 66374 , highlighted in green) are brighter and are found almost exclusively in the lowest LILr region. These forbidden transitions are peculiar because of their high-ionisation potential $(\geq 100 \mathrm{eV})$, pointing to a scenario in which the innermost parts of the outflowing gas in this galaxy are in optically thin, highly ionised regions, possibly directly illuminated by the central ionising AGN. 

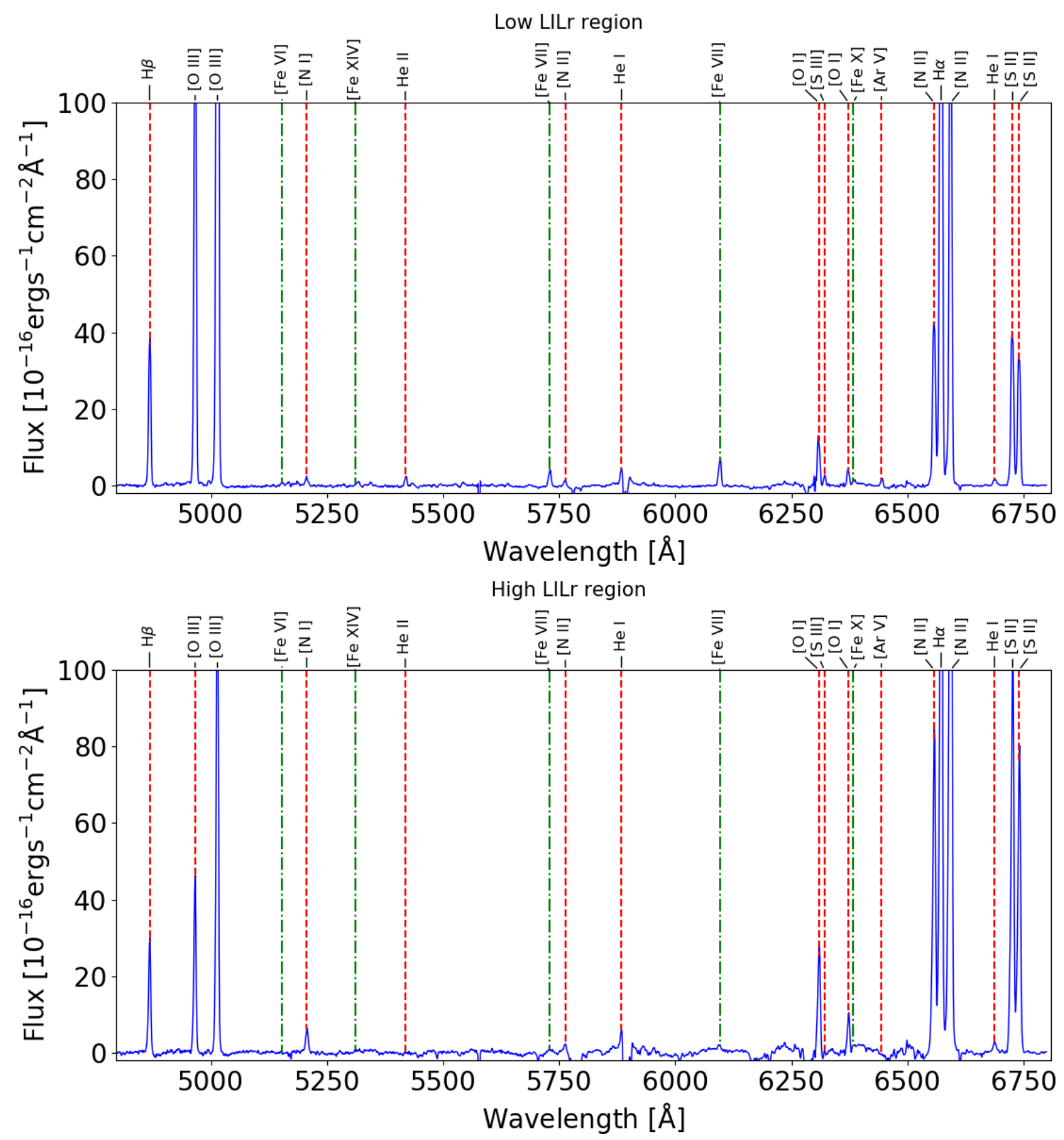

Fig. 7. Top panel: spectrum of the region characterised by $\log \left(\left[\mathrm{N}_{\mathrm{II}}\right] / \mathrm{H} \alpha\right)<-0.2$ and $\log \left(\left[\mathrm{S}_{\mathrm{II}}\right] / \mathrm{H} \alpha\right)<-0.4$ in Circinus, corresponding to the innermost regions of the outflowing cone. Bottom panel: spectrum of the region characterised by $\log \left(\left[\mathrm{N}_{\mathrm{II}}\right] / \mathrm{H} \alpha\right)>0.1$ and $\log \left(\left[\mathrm{S}_{\mathrm{II}}\right] / \mathrm{H} \alpha\right)>0$ in Circinus, mainly tracing the edges of the north-western outflowing cone. High-ionisation coronal lines (in green) are observed almost exclusively in the low LILr region, supporting a scenario where the inner parts of the outflowing gas in this galaxy are in optically thin highly ionised regions.

Furthermore, the $[\mathrm{N}$ II] $\lambda 5755$ auroral line, which is too faint to be detected in the single spaxel spectra, is observed in the stacked spectrum in Fig. 7. We can then determine the electron temperature $\left(T_{\mathrm{e}}\right)$ by exploiting the temperature-sensitive auroral-to-nebular line ratio of this particular ion. The atomic structure of $[\mathrm{N} \mathrm{II}]$ is such that auroral and nebular lines originate from excited states that are well spaced in energy, and thus their relative level populations depend heavily on electron temperature. From the $\left[\mathrm{N}_{\mathrm{II}}\right] \lambda \lambda 6548,84 /\left[\mathrm{N}_{\mathrm{II}}\right] \lambda 5755$ line ratio, we obtain $T_{\mathrm{e}} \sim 9.1 \times 10^{3} \mathrm{~K}$. This value is lower than the few other measurements present in the literature, to the best of our knowledge, derived in outflows by Villar Martín et al. (2014), Brusa et al. (2016), Perna et al. (2017), and Nesvadba (2018) (i.e. $T_{\mathrm{e}} \approx$ $\left.1.5 \times 10^{4} \mathrm{~K}\right)$. This discrepancy is possibly due to the fact that these authors estimated the electron temperature on the basis of [O III] (i.e. [O III] $\lambda \lambda 44959,5007 /\left[\mathrm{O}_{\text {III }}\right] \lambda 4363$ ), characterised by a higher ionisation potential with respect to [N II], which mainly traces the innermost parts of the photoionised regions and can be associated with lower temperatures (Osterbrock \& Ferland 2006; Curti et al. 2017; Perna et al., in prep.).

\subsection{Possible scenarios for the highest LILrs}

Figure 8 shows the $\left[\mathrm{N}_{\mathrm{II}}\right]-$ and $\left[\mathrm{S}_{\mathrm{II}}\right]-\mathrm{BPT}$ diagrams of all the MAGNUM galaxies (excluding NGC 1068 as before), colouredcoded as a function of the [O III] velocity dispersion $\sigma_{\text {[O }}$ III] as reported in the corresponding colour bars (darker shades means higher $\left.\sigma_{[\mathrm{O}} \mathrm{II}\right]$ ). Only bins from the Voronoi-binned cubes 
Table 1. Circinus: observed and dereddened line fluxes $(F$ and $I)$, relative to $\mathrm{H} \beta(\mathrm{H} \beta=1)$, and electron temperatures $T_{\mathrm{e}}$ for the low LILr $\left(\log ([\mathrm{N} I I] / H \alpha)<-0.2\right.$ and $\left.\log \left(\left[\mathrm{S}_{\mathrm{II}}\right] / \mathrm{H} \alpha\right)<-0.4\right)$ and high $\operatorname{LILr}(\log ([\mathrm{N} \mathrm{II}] / \mathrm{H} \alpha)>0.1$ and $\log ([\mathrm{S} \mathrm{II}] / \mathrm{H} \alpha)>0)$ regions, estimated by fitting the corresponding stacked spectra.

\begin{tabular}{|c|c|c|c|c|}
\hline & \multicolumn{2}{|c|}{ Low LILr region } & \multicolumn{2}{|c|}{ High LILr region } \\
\hline & $F$ & $I$ & $F$ & $I$ \\
\hline $\mathrm{H} \beta$ & 1 & 1 & 1 & 1 \\
\hline [O III] $] \lambda 5007$ & $11.14 \pm 0.04$ & $10.17 \pm 0.03$ & $4.69 \pm 0.02$ & $4.12 \pm 0.02$ \\
\hline$[\mathrm{Fe}$ vI $] \lambda 5146$ & $0.022 \pm 0.002$ & $0.018 \pm 0.002$ & - & - \\
\hline$[\mathrm{Fe}$ xIV $] \lambda 5303$ & $0.008 \pm 0.002$ & $0.006 \pm 0.002$ & - & - \\
\hline$[\mathrm{Fe}$ VII $] \lambda 5721$ & $0.210 \pm 0.002$ & $0.128 \pm 0.001$ & $0.098 \pm 0.004$ & $0.049 \pm 0.002$ \\
\hline$\left[\mathrm{N}_{\text {II }}\right] \lambda 5755$ & $0.034 \pm 0.002$ & $0.021 \pm 0.001$ & $0.093 \pm 0.004$ & $0.045 \pm 0.002$ \\
\hline$[\mathrm{Fe}$ VII $] \lambda 6087$ & $0.114 \pm 0.002$ & $0.058 \pm 0.001$ & $0.037 \pm 0.004$ & $0.014 \pm 0.001$ \\
\hline [O I $] \lambda 6300$ & $0.359 \pm 0.002$ & $0.171 \pm 0.001$ & $1.160 \pm 0.006$ & $0.402 \pm 0.001$ \\
\hline$\left[\mathrm{S}_{\text {III }}\right] \lambda 6312$ & $0.069 \pm 0.002$ & $0.033 \pm 0.001$ & - & - \\
\hline$[\mathrm{Fe} x] \lambda 6374$ & $0.041 \pm 0.002$ & $0.019 \pm 0.001$ & - & - \\
\hline$\left[\mathrm{N}_{\mathrm{II}}\right] \lambda 6584$ & $4.23 \pm 0.01$ & $1.765 \pm 0.002$ & $10.98 \pm 0.05$ & $3.168 \pm 0.002$ \\
\hline $\mathrm{H} \alpha$ & $6.79 \pm 0.02$ & 2.86 & $9.78 \pm 0.04$ & 2.86 \\
\hline$[\mathrm{S}$ II $] \lambda 6717$ & $1.141 \pm 0.005$ & $0.450 \pm 0.001$ & $3.46 \pm 0.02$ & $0.921 \pm 0.001$ \\
\hline$[\mathrm{S}$ II $] \lambda 6731$ & $1.357 \pm 0.005$ & $0.532 \pm 0.001$ & $4.62 \pm 0.02$ & $1.222 \pm 0.001$ \\
\hline $\mathrm{H} \beta$ flux & $227.2 \pm 0.7$ & $5160 \pm 40$ & $160.9 \pm 0.7$ & $13700 \pm 100$ \\
\hline$T_{\mathrm{e}}$ & \multicolumn{2}{|c|}{$(9.1 \pm 0.2) \times 10^{3}$} & \multicolumn{2}{|c|}{$(9.8 \pm 0.2) \times 10^{3}$} \\
\hline
\end{tabular}

Notes. Blank entries are undetected lines. $\mathrm{H} \beta$ flux is in units of $10^{-16} \mathrm{erg} \mathrm{s}^{-1} \mathrm{~cm}^{-2}$, while $T_{\mathrm{e}}$ is in Kelvin.

in which the [O III] line flux has a $S / N>3$ are shown. It can be clearly seen that the highest values of $\sigma_{[\mathrm{O} \text { II] }}$ correspond to the highest LILrs found in the outflowing gas. These mainly trace the edges of the outflowing cones and/or the region perpendicular to it (see Sect. 3.2). This correlation between velocity dispersion and emission-line ratio suggests that the kinematics and ionisation state are coupled, indicating that they may have the same physical origin. This trend has been already found in star-forming galaxies, U/LIRGs, and AGN (e.g. Monreal-Ibero et al. 2006, 2010; Rich et al. 2015, 2011; Ho et al. 2014; McElroy et al. 2015; Perna et al. 2017), and it was attributed to shocked gas components as photoionisation is not expected to cause such a trend (see Dopita \& Sutherland 1995 and the detailed discussion in McElroy et al. 2015).

Therefore, we compare the observed data with a grid of MAPPINGS III shock models ${ }^{6}$, taken from Allen et al. (2008), that comprises shock velocities in the range $v_{\mathrm{S}}=100-1000 \mathrm{~km} \mathrm{~s}^{-1}$ and magnetic parameters $B / n_{\mathrm{e}}{ }^{1 / 2}=10^{-2}-10 \mu \mathrm{Gcm}^{3 / 2}$, taking into account solar abundances and a pre-shock density $n_{\mathrm{e}} \sim 100 \mathrm{~cm}^{-3}$. It can be clearly seen that shock models can reproduce the high LILrs (up to values of $\sim 0.3$ ) that the MB-IB dichotomy could not explain. However, the models fail to reproduce the highest LILrs, possibly suggesting even more extreme conditions in the outflowing gas (see also Perna et al. 2017). We tested all the available Allen et al. (2008) shock models, but found no improvement. Since this discrepancy is more significant in the [N II]-BPT diagram, it may be partly related to a metallicity effect. Indeed, $\left[\mathrm{N}_{\mathrm{II}}\right] / \mathrm{H} \alpha$ line-ratios should increase with higher metallicity due to the secondary nitrogen production (e.g. Alloin et al. 1979; Considère et al. 2000; Mallery et al. 2007).

\footnotetext{
6 The values of the magnetic field, $B$, and magnetic parameter, $B / n_{\mathrm{e}}{ }^{1 / 2}$, were chosen by Allen et al. (2008) so as to cover the extremes expected in the ISM. Moreover, the shock velocities taken into account are consistent with those measured from the gas kinematics (Venturi et al., in prep.).
}

BPT diagrams colour-coded as a function of $\sigma_{\left[\mathrm{O}_{\text {III }}\right.}$ for each galaxy can be seen in Figs. D.7 and D.8, for [N II]- and [S II]-BPT diagrams, respectively. This correlation between [O III] velocity dispersion and high LILrs is visible especially in NGC 4945, NGC 5643, and IC 5063. Also NGC 1068 shows this trend, even though it shows far higher values of [O III] velocity dispersion in all the outflowing components. The fact that this correlation is not found in Circinus does not necessarily exclude the shock scenario because, as stressed in McElroy et al. (2015), for idealised planar shock fronts we would only be observing the shock at a single velocity, implying that the observed shock velocity and velocity dispersion will not necessarily have similar values.

From the $\left[\mathrm{N}_{\text {II }}\right] \lambda \lambda 6548,84 /\left[\mathrm{N}_{\text {II }}\right] \lambda 5755$ line ratio, measured from the stacked spectrum shown in the bottom panel of Fig. 7, we obtain $T_{\mathrm{e}} \sim 9.8 \times 10^{3} \mathrm{~K}$ instead of the much higher temperature that we should observe if the bulk of emission were associated with shock excitation $\left(T_{\mathrm{e}}>10^{5} \mathrm{~K}\right.$, Osterbrock \& Ferland 2006). A similar case has been found by Perna et al. (2017), who contend that a possible explanation could be that the shock accelerating the ISM gas is strongly cooled, so that the gas temperature rapidly returns to its pre-shock value (see also King 2014).

Nevertheless, some works pointed out the inefficiency of shocks in producing line emission. For example, Laor (1998) showed that shocks can reprocess only $\sim 10^{-6}$ of the rest mass to ionising radiation, with respect to a maximum conversion efficiency of $\sim 10^{-1}$ for the central continuum source, demonstrating that they can be a viable mechanism only in very low-luminosity sub-Eddington active galaxies, which are very inefficient in converting mass into radiation. An alternative explanation to account for the regions producing the strongest LILrs can be given by a hard ionising radiation field resulting from radiation filtered by clumpy, ionised absorbers located tens of parsecs or less from the nucleus, possibly consistent with the torus dimension (e.g. Netzer 2015). This scenario is consistent with our finding of lower $\left[\mathrm{S}_{\text {III }}\right] /\left[\mathrm{S}_{\text {II }}\right]$ line ratios $\left(\left[\mathrm{S}_{\mathrm{III}}\right] /\left[\mathrm{S}_{\mathrm{II}}\right]<\right.$ 1) in the regions characterised by the highest LILrs, with the exception of NGC 1068. Similar results have been obtained by 
[NII] BPT diagram
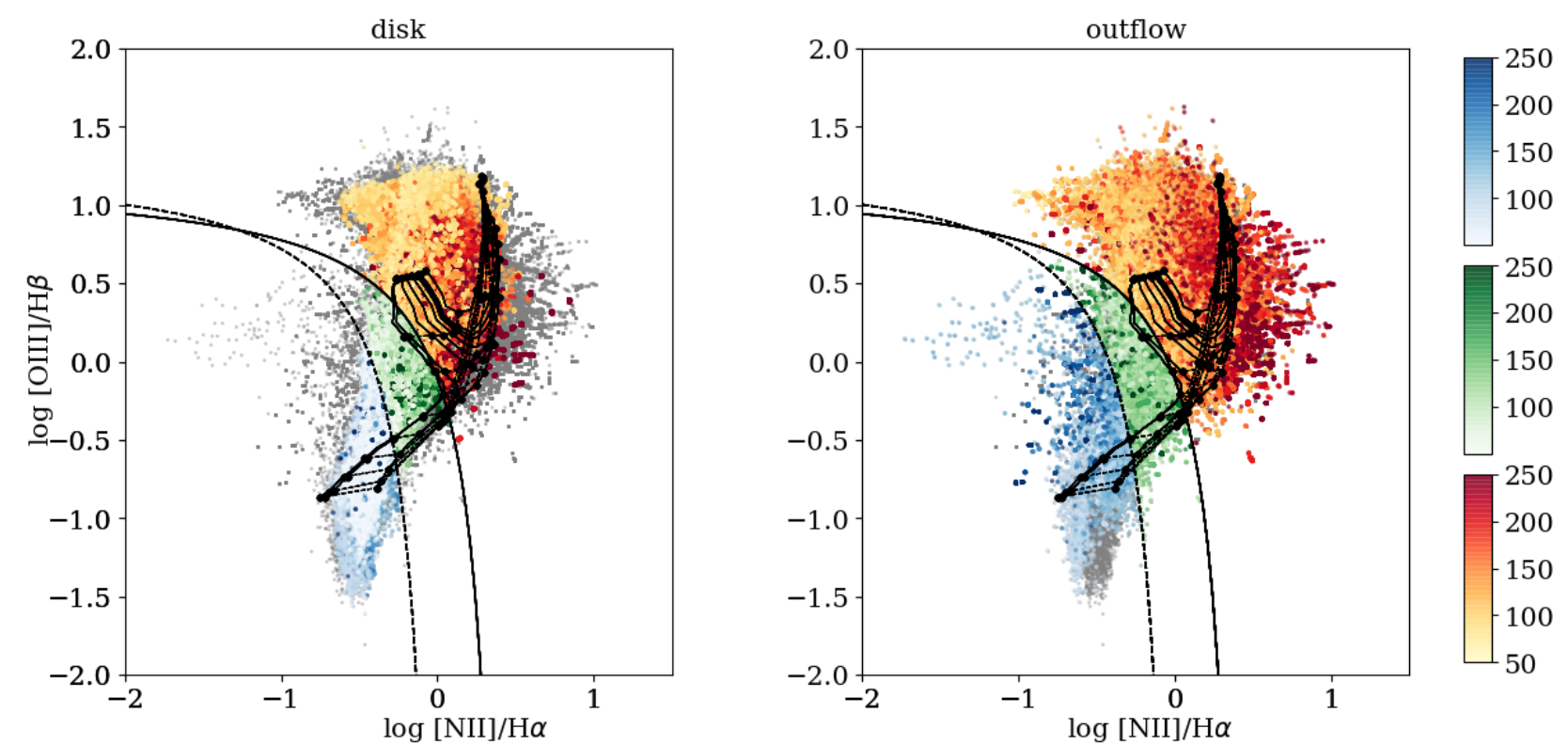

[SII] BPT diagram
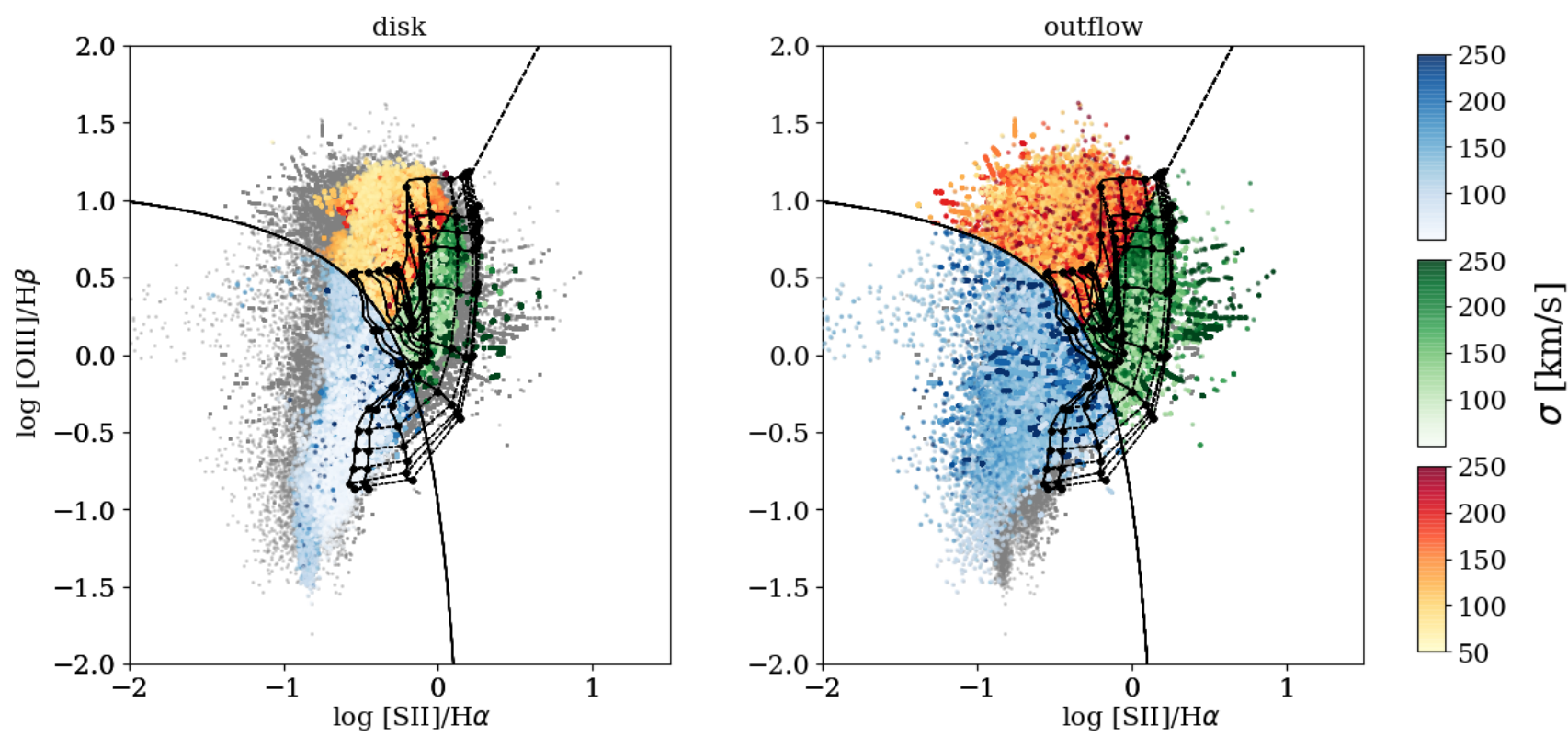

Fig. 8. [N II]- and [S II]-BPT diagrams for the disc and outflow components, as shown in Fig. 6, colour-coded as a function of the [O III] velocity dispersion (darker shades means higher $\sigma_{[\mathrm{Om}]}$ ). The grid of shock models taken from Allen et al. (2008) comprises shock velocities in the range $v_{\mathrm{S}}=100-1000 \mathrm{~km} \mathrm{~s}^{-1}$ (horizontally increasing) and magnetic parameters $B / n^{1 / 2}=10^{-2}-10 \mu \mathrm{G} \mathrm{cm}{ }^{3 / 2}$ (vertically increasing). The black dotted line is the Kewley et al. (2006) boundary between Seyferts and LI(N)ERs.

Kraemer et al. (2008), who analysed HST images of NGC 4151 and found that the $[\mathrm{O}$ III $] \lambda \lambda 4959,5007 /\left[\mathrm{O}_{\mathrm{II}}\right] \lambda \lambda 3726,29-$ a proxy of the ionisation parameter analogous to $\left[\mathrm{S}_{\mathrm{III}}\right] /\left[\mathrm{S}_{\mathrm{II}}\right]$ - is lower near the edges of the ionisation bicone observed in the galaxy than along its axis, concluding that the structure of the NLR is due to filtering of the ionising radiation by ionised gas, and is consistent with disc-wind models.

\section{Conclusions}

In this paper, we explored the gas properties (e.g. density, ionisation parameter, reddening and source of ionisation) of the outflowing gas in the (E)NLR of the 9 nearby Seyfert galaxies that are part of the MAGNUM survey, all characterised by promi- nent conical or biconical outflows. Exploiting the very high spatial resolution of the optical integral field MUSE spectrograph at VLT, we were able to disentangle the outflow component from the disc component in order to analyse its peculiarities through spatially and kinematically resolved maps. To do this, we divided the main emission lines ( $\left.\mathrm{H} \beta,\left[\mathrm{O}_{\mathrm{III}}\right],\left[\mathrm{O}_{\mathrm{I}}\right], \mathrm{H} \alpha, \mathrm{N}_{\mathrm{II}}\right],\left[\mathrm{S}_{\mathrm{II}}\right]$, and [S $\mathrm{SII}_{\mathrm{II}}$ ) in velocity bins, associating the core of the lines (centred on the stellar velocity in each spaxel) with the disc, and the blueshifted and redshifted wings with the outflow. In the following we report our main results:

- In Sect. 3.1, we show that the outflow component is characterised by higher median densities and $\left[\mathrm{S}_{\mathrm{III}}\right] /[\mathrm{S}$ II $]$ ratio, which is a proxy for the ionisation parameter $\left(\left\langle A_{V}\right\rangle \sim 0.9\right.$, 
$\left.\left\langle n_{\mathrm{e}}\right\rangle \sim 250 \mathrm{~cm}^{-3},\left\langle\log \left(\left[\mathrm{S}_{\mathrm{III}}\right] /\left[\mathrm{S}_{\mathrm{II}}\right]\right)\right\rangle \sim 0.16\right)$ with respect to the gas in the disc, which instead is more affected by dust extinction $\left(\left\langle A_{V}\right\rangle \sim 1.75,\left\langle n_{\mathrm{e}}\right\rangle \sim 130 \mathrm{~cm}^{-3},\left\langle\log \left(\left[\mathrm{S}_{\mathrm{III}}\right] /[\mathrm{S} \mathrm{II}]\right)\right\rangle \sim-0.38\right)$. Our median outflow density is lower with respect to what we found in the literature. However, calculating the median density weighting for the [S II] line flux, we obtain higher values $\left(\left\langle n_{\mathrm{e}}\right\rangle \sim 170 \mathrm{~cm}^{-3}\right.$ and $\left\langle n_{\mathrm{e}}\right\rangle \sim 815 \mathrm{~cm}^{-3}$, for disc and outflow, respectively). Therefore, many values of outflow density found in the literature could be biased towards higher $n_{\mathrm{e}}$ because they are based only on the most luminous outflowing regions and are characterised by a higher $\mathrm{S} / \mathrm{N}$.

- In Sect. 3.2, we analyse the ionisation state of the (E)NLR of MAGNUM galaxies, by making spatially and kinematically resolved BPT diagrams. We find that the AGN/LI(N)ERdominated outflow is characterised by the lowest and highest values of low-ionisation line ratios (LILrs, $\log ([\mathrm{N}$ II $] / \mathrm{H} \alpha) \sim-1$, $\log \left(\left[\mathrm{S}_{\mathrm{II}}\right] / \mathrm{H} \alpha\right) \sim-1$ and $\log \left(\left[\mathrm{N}_{\mathrm{II}}\right] / \mathrm{H} \alpha\right) \sim 0.5, \log \left(\left[\mathrm{S}_{\mathrm{II}}\right] / \mathrm{H} \alpha\right) \sim$ 0.5 , respectively), which are not observed in the disc. The lowest LILrs mainly come from the innermost regions of the outflowing cone, near the outflow axis, also characterised by the highest $\left[\mathrm{S}_{\mathrm{III}}\right] /\left[\mathrm{S}_{\mathrm{II}}\right]$ line ratios $\left(\left[\mathrm{S}_{\mathrm{III}}\right] /\left[\mathrm{S}_{\mathrm{II}}\right] \sim 2\right)$, meaning high excitation. On the other hand, the highest LILrs appear to come from the edges of the outflowing cones and/or from the regions perpendicular to the axis of the outflow, typically characterised by high values of $\left[\mathrm{O}_{\mathrm{III}}\right]$ velocity dispersion $\left(\sigma_{\left[\mathrm{O}_{\text {III }}\right.}>200 \mathrm{~km} \mathrm{~s}^{-1}\right)$.

- In Sect. 4, we make a comparison of our spatially and kinematically resolved BPT diagrams with photoionisation and shock models. Specifically, we found that the matter and ionisation bounded (MB and $\mathrm{IB}$ ) dichotomy first introduced by Binette et al. (1996) could reproduce well most of the observed features. MB clouds, characterised by high-ionisation lines (e.g. high $\left[\mathrm{O}_{\mathrm{III}}\right] / \mathrm{H} \beta$ ) and high excitation (i.e. high $\left[\mathrm{S}_{\mathrm{III}}\right] /\left[\mathrm{S}_{\mathrm{II}}\right]$ ) could account for both the $\left[\mathrm{N}\right.$ II] $/ \mathrm{H} \alpha,\left[\mathrm{S} \mathrm{II}_{\mathrm{II}} / \mathrm{H} \alpha\right.$ decrease and the $\left[\mathrm{S}_{\mathrm{III}}\right] /\left[\mathrm{S}_{\mathrm{II}}\right]$ enhancement observed in the majority of MAGNUM galaxies. On the other hand, IB clouds, optically thick and characterised by a much lower excitation, could be responsible for higher $\left[\mathrm{N}_{\mathrm{II}}\right] / \mathrm{H} \alpha$ and $\left[\mathrm{S}_{\mathrm{II}}\right] / \mathrm{H} \alpha$ line ratios, found both in the outflowing and in the disc components. Shocks may explain the highest $\left[\mathrm{N}_{\mathrm{II}}\right] / \mathrm{H} \alpha$ and $\left[\mathrm{S}_{\mathrm{II}}\right] / \mathrm{H} \alpha$ line ratios and the [O III] velocity dispersion enhancement. Consistently, we show the stacked spectrum of the region dominated by the lowest LILrs of Circinus, characterised by the presence of coronal lines

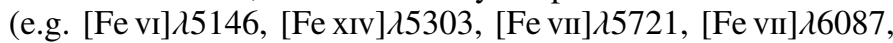
$[\mathrm{Fe} x] \lambda 6374)$, suggesting a very high excitation.

We speculate that the gas in the outflowing cones of our galaxies is set up in clumpy clouds characterised by higher density and ionisation parameters with respect to the disc gas. The innermost regions of the cone are optically thin to the radiation, being characterised by high excitation, and are possibly directly heated by the central ionising AGN. The edges of the cones and the regions perpendicular to the outflow axis could instead be dominated by shock excitation probably because of the interaction between the outflowing gas and the ISM. Alternatively, these regions, generally characterised by low excitation ([S $\mathrm{III}] /\left[\mathrm{S}_{\mathrm{II}}\right]<1$ ) could be impinged by an ionising radiation filtered by clumpy, ionised absorbers. Although these conclusions apply to the MAGNUM sample as a whole, some details may differ in individual galaxies. As an example, NGC1068 is the only galaxy that shows an enhancement of the $[\mathrm{S}$ III $] /\left[\mathrm{S}_{\mathrm{II}}\right]$ line ratio in the regions characterised by the highest LILrs. The next step of our analysis will be a detailed modelling of each MAGNUM galaxy, also making use of the photoionisation code Cloudy (Ferland et al. 2017) to better investigate the different scenarios that we propose to interpret our findings.
Acknowledgements. These results are based on observations collected at the European Southern Observatory under ESO programme 094.B-0321(A). MM acknowledges the University of California Santa Cruz (UCSC) for its stimulating scientific environment and is grateful to Carlo Cannarozzo for inspiring conversations and advice. GC acknowledges the support by INAF/Frontiera through the "Progetti Premiali" funding scheme of the Italian Ministry of Education, University, and Research. GC, FM, AG, PT, and SZ have been supported by the INAF PRIN-SKA 2017 programme 1.05.01.88.04. CC, CF, and EN acknowledge funding from the European Union's Horizon 2020 research and innovation programme under the Marie Skłodowska-Curie grant agreement No 664931. RM acknowledges ERC Advanced Grant 695671 "QUENCH" and support by the Science and Technology Facilities Council (STFC). This research has made use of NASA Astrophysics Data System and of the NASA/IPAC Extragalactic Database (NED), which is operated by the Jet Propulsion Laboratory, California Institute of Technology, under contract with the National Aeronautics and Space Administration.

\section{References}

Allen, M. G., Dopita, M. A., Tsvetanov, Z. I., \& Sutherland, R. S. 1999, ApJ, 511,686

Allen, M. G., Groves, B. A., Dopita, M. A., Sutherland, R. S., \& Kewley, L. J. 2008, ApJS, 178, 20

Allen, J. T., Croom, S. M., Konstantopoulos, I. S., et al. 2015, MNRAS, 446, 1567

Alloin, D., Collin-Souffrin, S., Joly, M., \& Vigroux, L. 1979, A\&A, 78, 200

Alonso-Herrero, A., Pereira-Santaella, M., García-Burillo, S., et al. 2018, ApJ, 859,144

Arribas, S., Colina, L., Bellocchi, E., Maiolino, R., \& Villar-Martín, M. 2014 A\&A, 568, A14

Axon, D. J., Marconi, A., Macchetto, F. D., Capetti, A., \& Robinson, A. 1997, Ap\&SS, 248, 69

Bacon, R., Accardo, M., Adjali, L., et al. 2010, in Ground basedand Airborne Instrumentation for Astronomy III, Proc. SPIE, 7735, 773508

Baldwin, J. A., Phillips, M. M., \& Terlevich, R. 1981, PASP, 93, 5

Barbosa, F. K. B., Storchi-Bergmann, T., McGregor, P., Vale, T. B., \& Rogemar Riffel, A. 2014, MNRAS, 445, 2353

Baumgartner, W. H., Tueller, J., Markwardt, C. B., et al. 2013, ApJS, 207, 19

Belfiore, F., Maiolino, R., Maraston, C., et al. 2016, MNRAS, 461, 3111

Bian, F., Kewley, L. J., Dopita, M. A., \& Juneau, S. 2016, ApJ, 822, 62

Binette, L., Wilson, A. S., \& Storchi-Bergmann, T. 1996, A\&A, 312, 365

Blanco, V. M., Graham, J. A., Lasker, B. M., \& Osmer, P. S. 1975, ApJ, 198, L63

Bland-Hawthorn, J., \& Kedziora-Chudczer, L. 2003, PASA, 20, 242

Bland-Hawthorn, J., Lumsden, S. L., Voit, G. M., Cecil, G. N., \& Weisheit, J. C. 1997, Ap\&SS, 248, 177

Bruhweiler, F. C., Truong, K. Q., \& Altner, B. 1991, ApJ, 379, 596

Brusa, M., Perna, M., Cresci, G., et al. 2016, A\&A, 588, A58

Bundy, K., Bershady, M. A., Law, D. R., et al. 2015, ApJ, 798, 7

Calzetti, D., Armus, L., Bohlin, R. C., et al. 2000, ApJ, 533, 682

Cano-Díaz, M., Maiolino, R., Marconi, A., et al. 2012, A\&A, 537, L8

Cappellari, M., \& Copin, Y. 2003, MNRAS, 342, 345

Cappellari, M., \& Emsellem, E. 2004, PASP, 116, 138

Carniani, S., Marconi, A., Maiolino, R., et al. 2016, A\&A, 591, A28

Cecil, G., Bland, J., \& Tully, R. B. 1990, ApJ, 355, 70

Cecil, G., Dopita, M. A., Groves, B., et al. 2002, ApJ, 568, 627

Colbert, E. J. M., Baum, S. A., Gallimore, J. F., O'Dea, C. P., \& Christensen, J. A. 1996a, ApJ, 467, 551

Colbert, E. J. M., Baum, S. A., Gallimore, J. F., et al. 1996b, ApJS, 105, 75

Colina, L., Fricke, K. J., Kollatschny, W., \& Perryman, M. A. C. 1987, A\&A, 178,51

Colina, L., Sparks, W. B., \& Macchetto, F. 1991, ApJ, 370, 102

Considère, S., Coziol, R., Contini, T., \& Davoust, E. 2000, A\&A, 356, 89

Contini, T., Garilli, B., Le Fèvre, O., et al. 2012, A\&A, 539, A91

Crenshaw, D. M., \& Kraemer, S. B. 2000, ApJ, 532, L101

Crenshaw, D. M., Kraemer, S. B., Schmitt, H. R., et al. 2010, AJ, 139, 871

Cresci, G., \& Maiolino, R. 2018, Nat. Astron., 2, 179

Cresci, G., Vanzi, L., Sauvage, M., Santangelo, G., \& van der Werf, P. 2010, A\&A, 520, A82

Cresci, G., Marconi, A., Zibetti, S., et al. 2015a, A\&A, 582, A63

Cresci, G., Mainieri, V., Brusa, M., et al. 2015b, ApJ, 799, 82

Curti, M., Cresci, G., Mannucci, F., et al. 2017, MNRAS, 465, 1384

Das, V., Crenshaw, D. M., Kraemer, S. B., \& Deo, R. P. 2006, AJ, 132, 620

Dasyra, K. M., Bostrom, A. C., Combes, F., \& Vlahakis, N. 2015, ApJ, 815, 34

Díaz, A. I., Castellanos, M., Terlevich, E., \& Luisa García-Vargas, M. 2000,

MNRAS, 318, 462

Dopita, M. A., \& Sutherland, R. S. 1995, ApJ, 455, 468

Dopita, M. A., \& Sutherland, R. S. 1996, ApJS, 102, 161 
Dopita, M. A., Fischera, J., Sutherland, R. S., et al. 2006, ApJ, 647, 244

Fabian, A. C. 2012, ARA\&A, 50, 455

Ferguson, J. W., Korista, K. T., Baldwin, J. A., \& Ferland, G. J. 1997, ApJ, 487, 122

Ferland, G. J., Chatzikos, M., Guzmán, F., et al. 2017, Rev. Mex. Astron. Astrofis., 53, 385

Ferruit, P., Wilson, A. S., \& Mulchaey, J. 2000, ApJS, 128, 139

Fischer, T. C., Crenshaw, D. M., Kraemer, S. B., \& Schmitt, H. R. 2013, ApJS 209, 1

Förster Schreiber, N. M., Genzel, R., Bouché, N., et al. 2009, ApJ, 706, 1364

Förster Schreiber, N. M., Renzini, A., Mancini, C., et al. 2018a, ApJS, 238, 21

Förster Schreiber, N.M., Übler, H., Davies, R.L., et al. 2018b, ApJ, submitted [arXiv: 1807.04738]

Friedrich, S., Davies, R. I., Hicks, E. K. S., et al. 2010, A\&A, 519, A79

Gallimore, J. F., Baum, S. A., O’Dea, C. P., Brinks, E., \& Pedlar, A. 1994, ApJ, 422, L13

Gallimore, J. F., Elitzur, M., Maiolino, R., et al. 2016, ApJ, 829, L7

García-Burillo, S., Combes, F., Usero, A., et al. 2014, A\&A, 567, A125

Greene, J. E., Zakamska, N. L., \& Smith, P. S. 2012, ApJ, 746, 86

Greenhill, L. J., Kondratko, P. T., Lovell, J. E. J., et al. 2003, ApJ, 582, L11

Guainazzi, M., Matt, G., Brandt, W. N., et al. 2000, A\&A, 356, 463

Hardcastle, M. J., Worrall, D. M., Kraft, R. P., et al. 2003, ApJ, 593, 169

Harrison, C. M., Alexander, D. M., Mullaney, J. R., et al. 2016, MNRAS, 456 1195

Heckman, T. M. 1980, A\&A, 87, 152

Ho, L. C., Li, Z.-Y., Barth, A. J., Seigar, M. S., \& Peng, C. Y. 2011, ApJS, 197, 21

Ho, I.-T., Kewley, L. J., Dopita, M. A., et al. 2014, MNRAS, 444, 3894

Holt, J., Tadhunter, C. N., Morganti, R., \& Emonts, B. H. C. 2011, MNRAS, 410, 1527

Hutchings, J. B., Crenshaw, D. M., Kaiser, M. E., et al. 1998, ApJ, 492, L115

Kakkad, D., Groves, B., Dopita, M., et al. 2018, A\&A, 618, A6

Karouzos, M., Woo, J.-H., \& Bae, H.-J. 2016a, ApJ, 819, 148

Karouzos, M., Woo, J.-H., \& Bae, H.-J. 2016b, ApJ, 833, 171

Kauffmann, G., Heckman, T. M., Tremonti, C., et al. 2003, MNRAS, 346, 1055

Kewley, L. J., \& Dopita, M. A. 2002, ApJS, 142, 35

Kewley, L. J., \& Ellison, S. L. 2008, ApJ, 681, 1183

Kewley, L. J., Dopita, M. A., Sutherland, R. S., Heisler, C. A., \& Trevena, J. 2001, ApJ, 556, 121

Kewley, L. J., Groves, B., Kauffmann, G., \& Heckman, T. 2006, MNRAS, 372, 961

King, A. 2014, Space Sci. Rev., 183, 427

Komossa, S., \& Schulz, H. 1997, A\&A, 323, 31

Kraemer, S. B., Schmitt, H. R., \& Crenshaw, D. M. 2008, ApJ, 679, 1128

Kraft, R. P., Hardcastle, M. J., Sivakoff, G. R., et al. 2008, ApJ, 677, L97

Laor, A. 1998, ApJ, 496, L71

Law, D. R., Steidel, C. C., Erb, D. K., et al. 2009, ApJ, 697, 2057

Lena, D., Robinson, A., Storchi-Bergman, T., et al. 2015, ApJ, 806, 84

Lester, D. F., Joy, M., Harvey, P. M., Ellis, Jr., H. B., \& Parmar, P. S. 1987, ApJ, 321,755

Liang, Y. C., Yin, S. Y., Hammer, F., et al. 2006, ApJ, 652, 257

Lindblad, P. O. 1999, A\&ARv, 9, 221

Liu, C., Yuan, F., Ostriker, J. P., Gan, Z., \& Yang, X. 2013, MNRAS, 434, 1721

Macchetto, F., Capetti, A., Sparks, W. B., Axon, D. J., \& Boksenberg, A. 1994, ApJ, 435, L15

Maiolino, R., \& Rieke, G. H. 1995, ApJ, 454, 95

Mallery, R. P., Kewley, L., Rich, R. M., et al. 2007, ApJS, 173, 482

Marconi, A., Moorwood, A. F. M., Origlia, L., \& Oliva, E. 1994, The Messenger, 78,20

Marconi, A., Oliva, E., van der Werf, P. P., et al. 2000, A\&A, 357, 24

Markwardt, C. B. 2009, in Astronomical Data Analysis Software and Systems

XVIII, eds. D. A. Bohlender, \& P. Dowler, ASP Conf. Ser., 411, 251

Matt, G., Fabian, A. C., Guainazzi, M., et al. 2000, MNRAS, 318, 173

McElroy, R., Croom, S. M., Pracy, M., et al. 2015, MNRAS, 446, 2186

McLeod, A. F., Dale, J. E., Ginsburg, A., et al. 2015, MNRAS, 450, 1057

Monreal-Ibero, A., Arribas, S., \& Colina, L. 2006, ApJ, 637, 138
Monreal-Ibero, A., Arribas, S., Colina, L., et al. 2010, A\&A, 517, A28

Moorwood, A. F. M., Lutz, D., Oliva, E., et al. 1996, A\&A, 315, L109

Morganti, R. 2010, PASA, 27, 463

Morganti, R., Oosterloo, T., \& Tsvetanov, Z. 1998, AJ, 115, 915

Morisset, C., Delgado-Inglada, G., Sánchez, S. F., et al. 2016, A\&A, 594, A37

Nakajima, K., \& Ouchi, M. 2014, MNRAS, 442, 900

Nesvadba, N. 2018, Am. Astron. Soc. Meet. Abstr., 231, 125.08

Netzer, H. 2015, ARA\&A, 53, 365

Oliva, E., Salvati, M., Moorwood, A. F. M., \& Marconi, A. 1994, A\&A, 288, 457

Oosterloo, T., Raymond Oonk, J. B., Morganti, R., et al. 2017, A\&A, 608, A38

Osterbrock, D. E., \& Ferland, G. J. 2006, Astrophysics of Gaseous Nebulae and Active Galactic Nuclei (Mill Valley, CA: University Science Books)

Pérez-Beaupuits, J. P., Spoon, H. W. W., Spaans, M., \& Smith, J. D. 2011, A\&A, 533, A56

Perna, M., Brusa, M., Salvato, M., et al. 2015, A\&A, 583, A72

Perna, M., Lanzuisi, G., Brusa, M., Cresci, G., \& Mignoli, M. 2017, A\&A, 606, A96

Rich, J. A., Kewley, L. J., \& Dopita, M. A. 2011, ApJ, 734, 87

Rich, J. A., Kewley, L. J., \& Dopita, M. A. 2015, ApJS, 221, 28

Riffel, R. A., Storchi-Bergmann, T., \& Winge, C. 2013, MNRAS, 430, 2249

Risaliti, G., Maiolino, R., \& Salvati, M. 1999, ApJ, 522, 157

Sánchez, S. F., Kennicutt, R. C., Gil de Paz, A., et al. 2012, A\&A, 538, A8

Schmitt, H. R., Storchi-Bergmann, T., \& Baldwin, J. A. 1994, ApJ, 423, 237

Scoville, N. Z., Matthews, K., Carico, D. P., \& Sanders, D. B. 1988, ApJ, 327, L61

Sharp, R. G., \& Bland-Hawthorn, J. 2010, ApJ, 711, 818

Silk, J. 2013, ApJ, 772, 112

Silk, J., \& Rees, M. J. 1998, A\&A, 331, L1

Simpson, C., Wilson, A. S., Bower, G., et al. 1997, ApJ, 474, 121

Singh, R., van de Ven, G., Jahnke, K., et al. 2013, A\&A, 558, A43

Stalevski, M., Asmus, D., \& Tristram, K. R. W. 2017, MNRAS, 472, 3854

Storchi-Bergmann, T., Lopes, R. D. S., McGregor, P. J., et al. 2010, MNRAS, 402, 819

Storchi-Bergmann, T., Wilson, A. S., \& Baldwin, J. A. 1992, ApJ, 396, 45

Stott, J. P., Swinbank, A. M., Johnson, H. L., et al. 2016, MNRAS, 457, 1888

Sun, A.-L., Greene, J. E., \& Zakamska, N. L. 2017, ApJ, 835, 222

Sun, A.-L., Greene, J. E., Zakamska, N. L., et al. 2018, MNRAS, 480, 2302

Tadhunter, C., Morganti, R., Rose, M., Oonk, J. B. R., \& Oosterloo, T. 2014, Nature, 511, 440

Thronson, Jr., H. A., Hereld, M., Majewski, S., et al. 1989, ApJ, 343, 158

Trippe, M. L., Crenshaw, D. M., Deo, R., \& Dietrich, M. 2008, AJ, 135, 2048

Turner, O. J., Cirasuolo, M., Harrison, C. M., et al. 2017, MNRAS, 471, 1280

Vaona, L., Ciroi, S., Di Mille, F., et al. 2012, MNRAS, 427, 1266

Vazdekis, A., Sánchez-Blázquez, P., Falcón-Barroso, J., et al. 2010, MNRAS, 404, 1639

Veilleux, S., \& Bland-Hawthorn, J. 1997, ApJ, 479, L105

Veilleux, S., \& Osterbrock, D. E. 1987, ApJS, 63, 295

Veilleux, S., Shopbell, P. L., \& Miller, S. T. 2001, AJ, 121, 198

Venturi, G., Marconi, A., Mingozzi, M., et al. 2017, Front. Astron. Space Sci., 4, 46

Venturi, G., Nardini, E., Marconi, A., et al. 2018, A\&A, 619, A74

Vilchez, J. M., \& Esteban, C. 1996, MNRAS, 280, 720

Villar Martín, M., Emonts, B., Humphrey, A., Cabrera Lavers, A., \& Binette, L. 2014, MNRAS, 440, 3202

Villar Martín, M., Bellocchi, E., Stern, J., et al. 2015, MNRAS, 454, 439

Ward, M., Penston, M. V., Blades, J. C., \& Turtle, A. J. 1980, MNRAS, 193, 563

Westmoquette, M. S., Smith, L. J., \& Gallagher, III., J. S. 2011, MNRAS, 414, 3719

Westmoquette, M. S., Clements, D. L., Bendo, G. J., \& Khan, S. A. 2012, MNRAS, 424, 416

Westmoquette, M. S., Dale, J. E., Ercolano, B., \& Smith, L. J. 2013, MNRAS, 435,30

Wisnioski, E., Förster Schreiber, N. M., Wuyts, S., et al. 2015, ApJ, 799, 209

Zschaechner, L. K., Walter, F., Bolatto, A., et al. 2016, ApJ, 832, 142 
M. Mingozzi et al.: Gas properties in the outflowing and disc components in nearby active galaxies with MUSE

\section{Appendix A: Extinction maps}

We derive the dust extinction map for each galaxy, making use of the Balmer decrement $\mathrm{H} \alpha / \mathrm{H} \beta$, assuming a Calzetti et al. (2000) attenuation law and a fixed temperature of $10^{4} \mathrm{~K}$. The resulting extinction map for the MAGNUM galaxies Centaurus A, IC 5063, NGC 1068, NGC 1365, NGC 1386, NGC 2992, NGC 4945, and NGC 5643 are shown in Fig. A.1. We show only the spaxels in which the $\mathrm{H} \alpha$ and $\mathrm{H} \beta$ lines are detected with $S / N>5$.
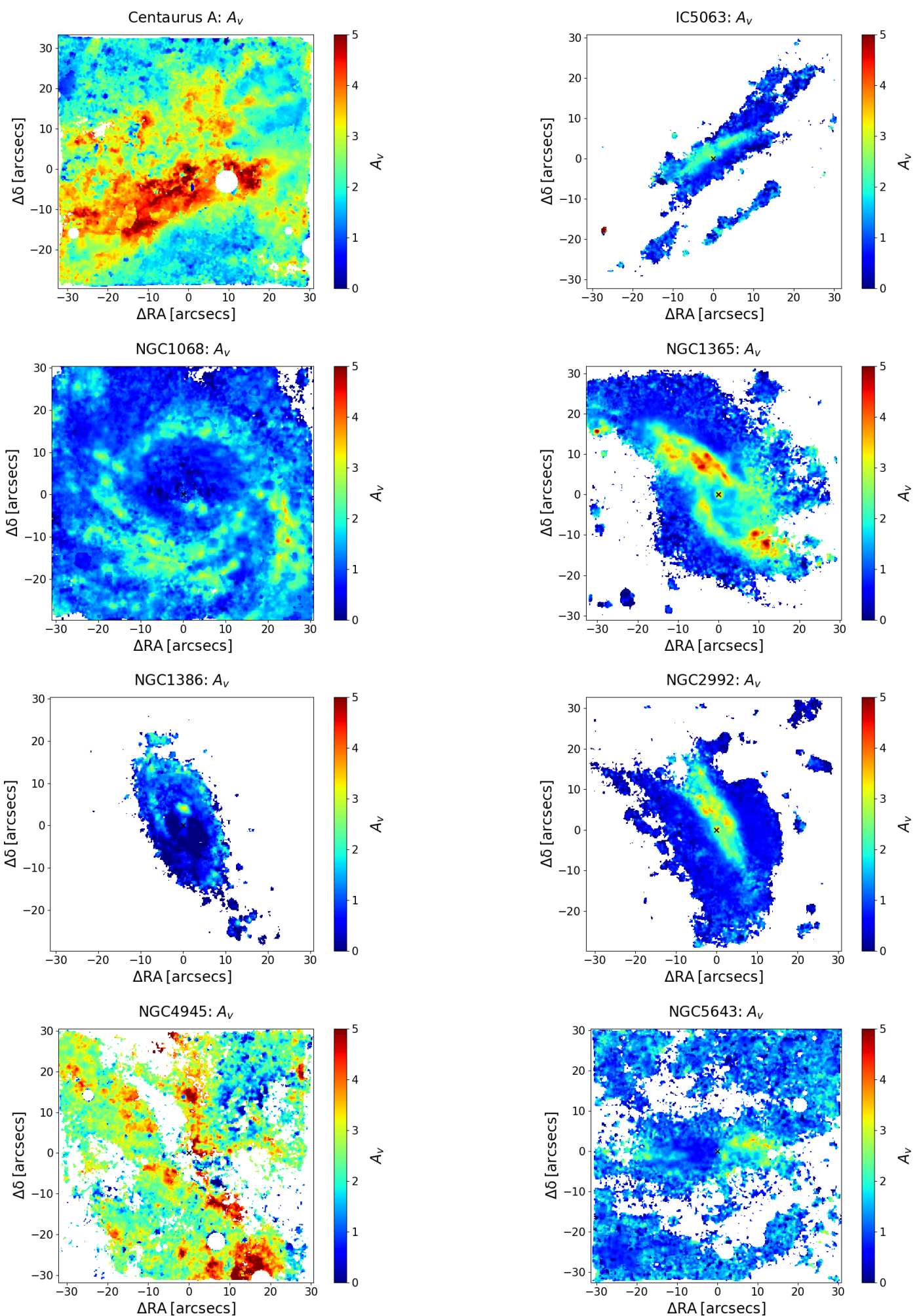

Fig. A.1. Maps of the total extinction in $V$ band $A_{V}$, obtained from the Balmer decrement $\mathrm{H} \alpha / \mathrm{H} \beta$, for Centaurus A, IC 5063, NGC 1068, NGC 1365 , NGC 1386, NGC 2992, NGC 4945, and NGC 5643. Only spaxels with $\mathrm{H} \alpha$ and $\mathrm{H} \beta S / N>5$ are shown. 


\section{Appendix B: Electron density maps}

We have computed the $\left[\mathrm{S}_{\mathrm{II}}\right] \lambda \lambda 6717,31$ line ratio in each spaxel where the [S II] lines are detected with $S / N>5$, and converted it to an electron density using the Osterbrock \& Ferland
(2006) model, sensible to the density variations in the range $50 \mathrm{~cm}^{-3}<n_{\mathrm{e}}<5000 \mathrm{~cm}^{-3}$, assuming a temperature of $10^{4} \mathrm{~K}$. The resulting electron density maps of the MAGNUM galaxies Centaurus A, IC 5063, NGC 1068, NGC 1365, NGC 1386, NGC 2992, NGC 4945, and NGC 5643 are shown in Fig. B.1.
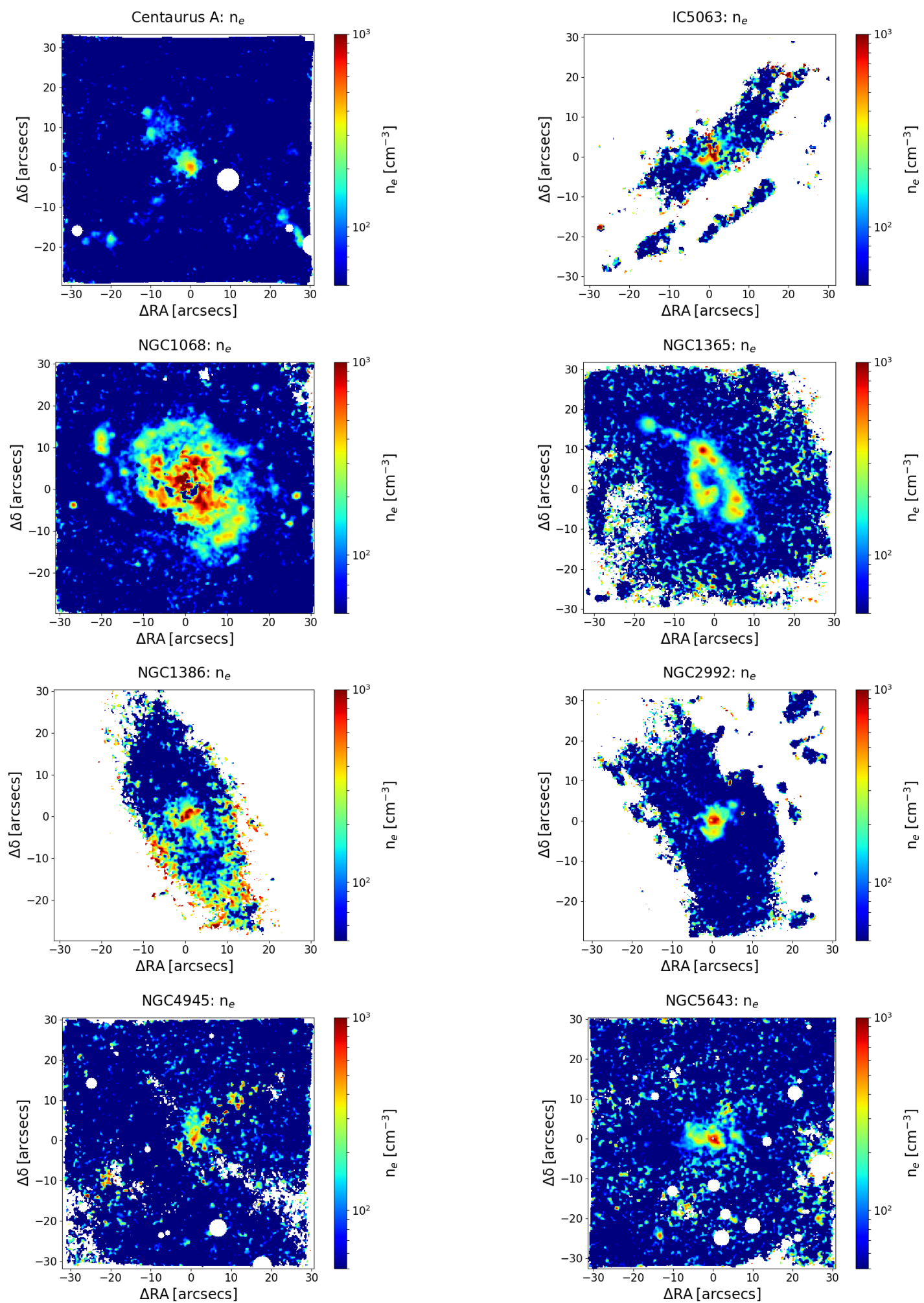

Fig. B.1. Maps of the total electron density $n_{\mathrm{e}}$, measured from the $\left[\mathrm{S}_{\mathrm{II}}\right] \lambda 6717 /\left[\mathrm{S}_{\mathrm{II}}\right] \lambda 6731$ ratio, for Centaurus A, IC 5063, NGC 1068, NGC 1365,

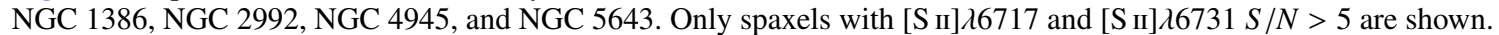


M. Mingozzi et al.: Gas properties in the outflowing and disc components in nearby active galaxies with MUSE

\section{Appendix C: [S III]/[SII maps}

We computed the $\left[\mathrm{S}_{\mathrm{III}}\right] \lambda \lambda 9069,9532 /\left[\mathrm{S}_{\mathrm{II}}\right] \lambda \lambda 6717,31$ line ratio in each MUSE spaxel where the line fluxes are detected with
$S / N>5$ since it is a proxy for the ionisation parameter (e.g. Díaz et al. 2000). The resulting maps for the MAGNUM galaxies Centaurus A, IC 5063, NGC 1068, NGC 1365, NGC 1386, NGC 2992, NGC 4945, and NGC 5643 are shown in Fig. C.1.
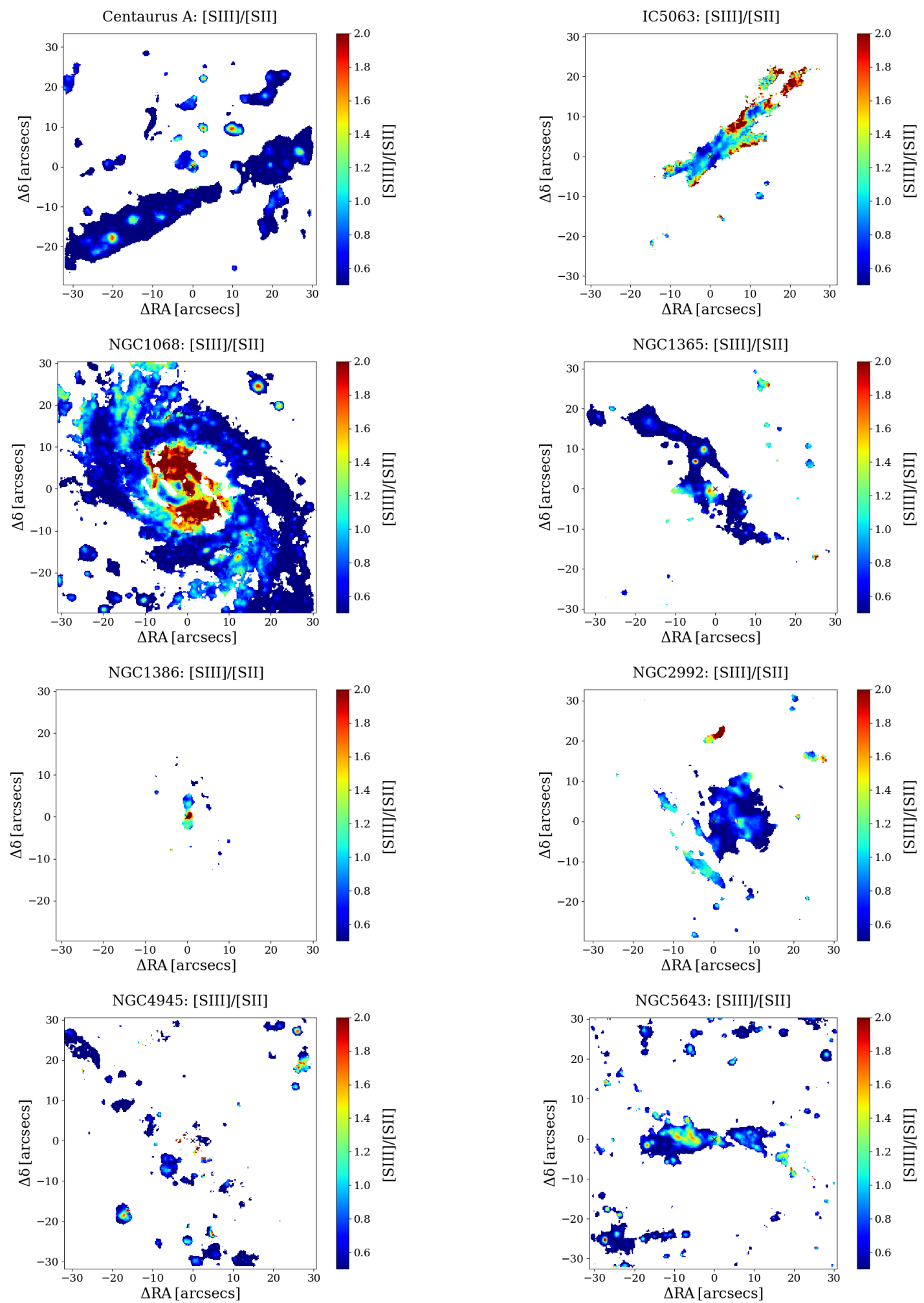

Fig. C.1. Maps of [S III] $\lambda \lambda 9069,9532 /\left[\mathrm{S}_{\mathrm{II}}\right] \lambda \lambda 6717,31$ ratio, a proxy for the ionisation parameter, for Centaurus A, IC 5063, NGC 1068, NGC 1365, NGC 1386, NGC 2992, NGC 4945, and NGC 5643. Only spaxels with [S $\left.\mathrm{III}_{\mathrm{II}}\right] \lambda 9069$ and [S $\left.\mathrm{II}\right] \lambda \lambda 6717,31 S / N>5$ are shown. 


\section{Appendix D: Spatially and kinematically resolved BPT diagrams}

The left panels of Figs. D.1-D.4 show the [N II]- and [S II]BPT diagrams for the disc and outflow components of Centaurus A, Circinus, IC 5063, NGC 1068, NGC 1365, NGC 1386, NGC 2992, NGC 4945, and NGC 5643, respectively. The dashed curve is the boundary between starforming galaxies and AGN defined by Kauffmann et al. (2003), while the solid curve is the theoretical upper limit on SF line ratios found by Kewley et al. (2001). The dotted line, instead, is the boundary between Seyferts and LI(N)ERs introduced by Kewley et al. (2006). The dominant source of ionisation is colour-coded: blue for SF, green for intermediate regions in the $[\mathrm{NII}]-\mathrm{BPT}$ and $\mathrm{LI}(\mathrm{N}) \mathrm{ER}$ in the $\left[\mathrm{S}_{\mathrm{II}}\right]-\mathrm{BPT}$, and red for AGN-like ionised spectra. The colours are shaded as a function of the $x$-axis line ratios (darker shades means higher $x$-axis line ratios). The corresponding position on the map of the galaxy, colour-coded according to the different source of ionisation, is shown in the right panels. In the background of all the pictures (black dots in the BPTs and shaded grey in the corresponding maps) are reported the disc and outflow components together to allow a better visual comparison.

Moreover, Figs. D.5 and D.6 show the [N II]- and [S II]-BPT diagrams colour-coded as a function of the $\left[\mathrm{S}_{\mathrm{III}}\right] /\left[\mathrm{S}_{\mathrm{II}}\right]$ line ratio, which is a proxy of the ionisation parameter (darker shades means higher $[\mathrm{S} \mathrm{III}] /\left[\mathrm{S}_{\mathrm{II}}\right]$ line ratios). Finally, Figs. D.7 and D.8 show the [N $\left.\mathrm{N}_{\mathrm{II}}\right]$ - and [ $\left.\mathrm{S}_{\mathrm{II}}\right]$-BPT diagrams colour-coded as a function of the [O III] velocity dispersion $\sigma_{[\mathrm{O} \text { III] }}$ (darker shades means higher $\sigma_{[\mathrm{O} \text { III }]}$ ).

For each velocity bin, we selected only the spaxels with a $S / N>5$ on each emission line involved in the given diagram, computed by dividing the flux integrated in the velocity channels considered by the corresponding noise.

For NGC 1365, we show the BPT diagrams obtained from the Voronoi-binned cube in order to detect the biconical outflow, which would be too weak to be detected in $\mathrm{H} \beta$ otherwise. 
M. Mingozzi et al.: Gas properties in the outflowing and disc components in nearby active galaxies with MUSE
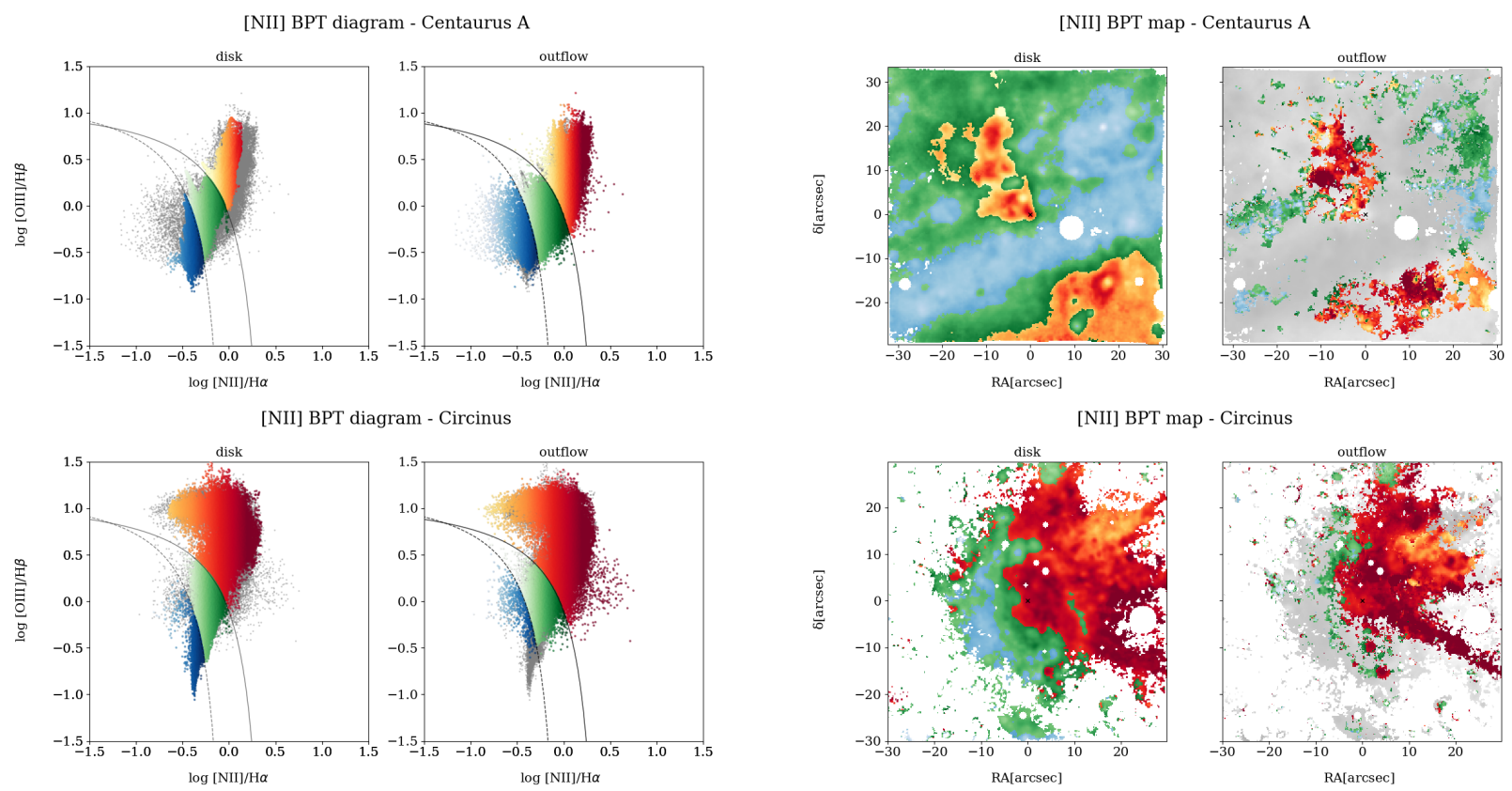

[NII] BPT diagram - IC5063
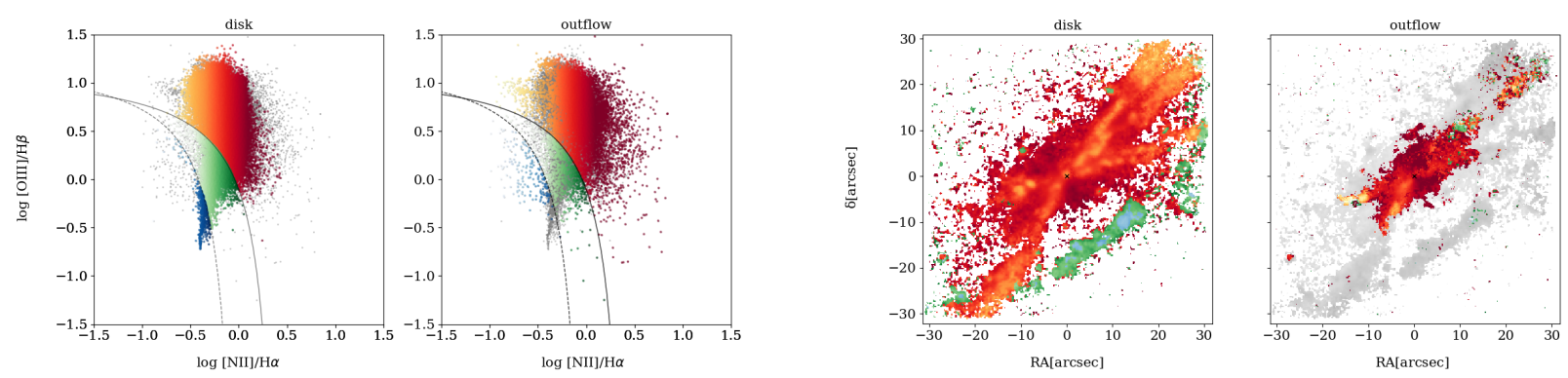

[NII] BPT diagram - NGC1068
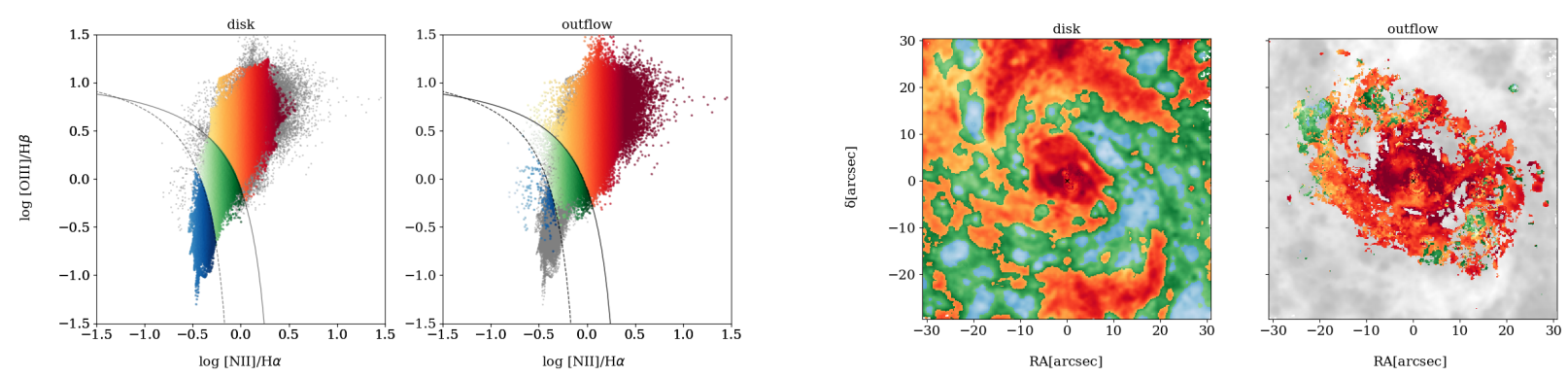

[NII] BPT diagram - NGC1365
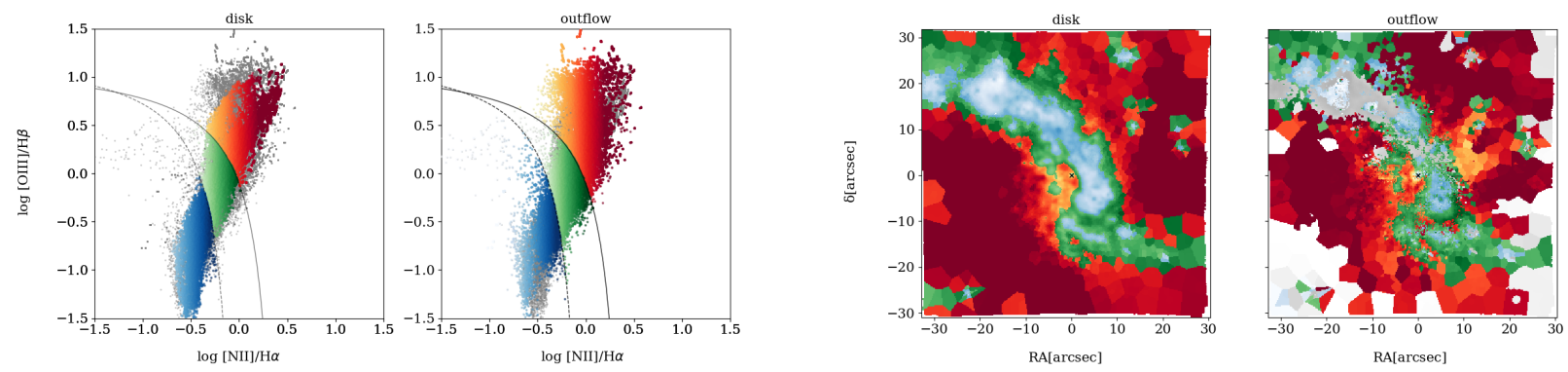

Fig. D.1. Left panels: [N II]-BPT diagrams for the disc and outflow components, on the left and the right respectively, of Centaurus A, IC 5063, NGC 1068, and NGC 1365, colour-coded as follows: shades of blue for SF, green for composite regions, and red for AGN-like ionising spectra, as a function of the $[\mathrm{N} \mathrm{II}] / \mathrm{H} \alpha$ line ratio (darker shades means higher $[\mathrm{N} \mathrm{II}] / \mathrm{H} \alpha$ ). The black dashed curve is the boundary between star-forming galaxies and AGN defined by Kauffmann et al. (2003), while the black solid curve is the theoretical upper limit on SF line ratios found by Kewley et al. (2001). Right panels: [N II]-BPT maps, colour-coded according to the BPT classification. In the background of all the pictures (black dots in the BPTs and shaded grey in the corresponding maps), are shown the disc and outflow component together. For each velocity bin, we select only the spaxels with a $S / N>5$ for all the flux line ratios. 
[NII] BPT diagram - NGC1386
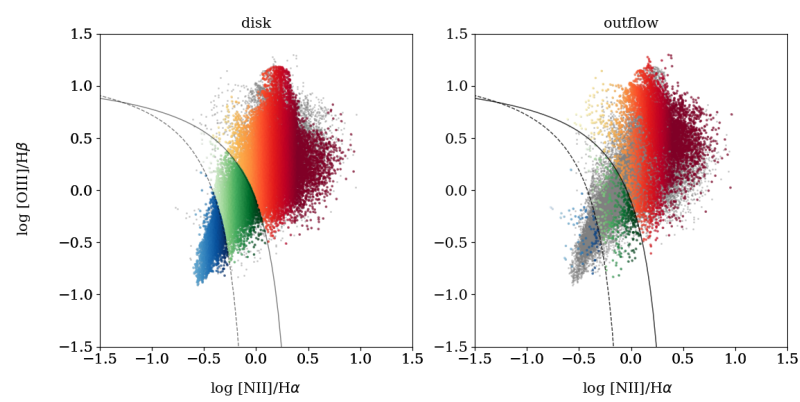

[NII] BPT diagram - NGC2992
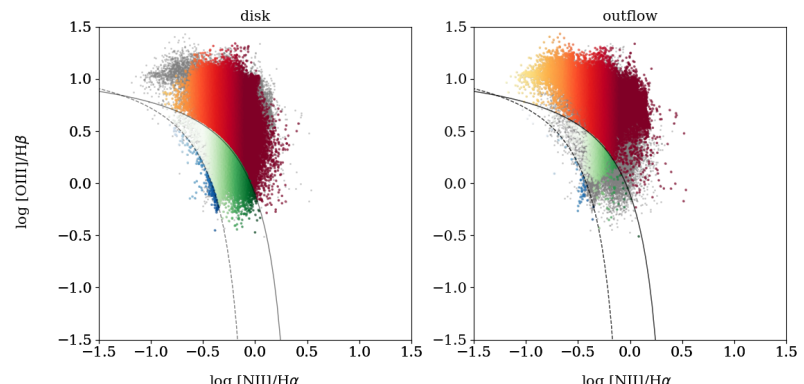

[NII] BPT diagram - NGC4945
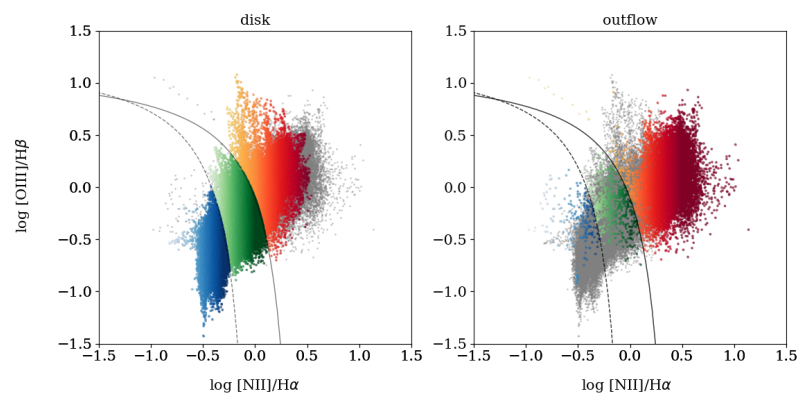

[NII] BPT diagram - NGC5643
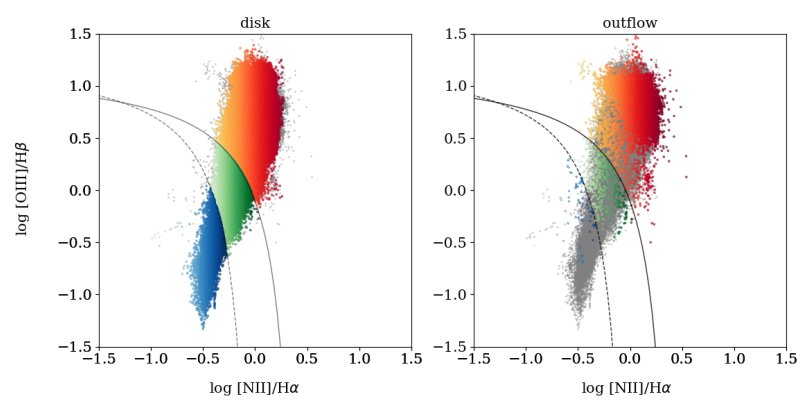

[NII] BPT map - NGC1386
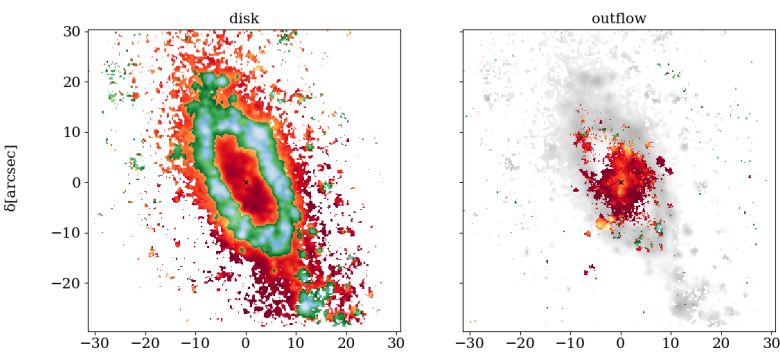

[NII] BPT map - NGC2992
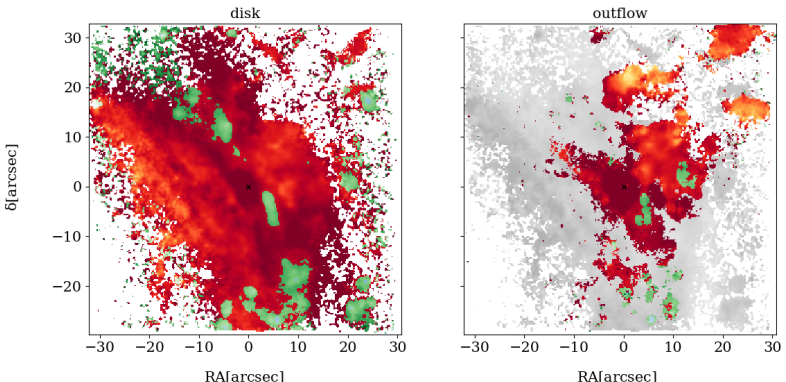

[NII] BPT map - NGC4945
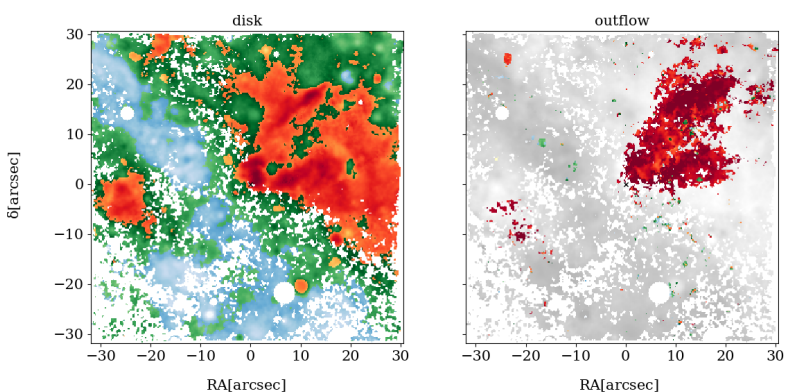

[NII] BPT map - NGC5643
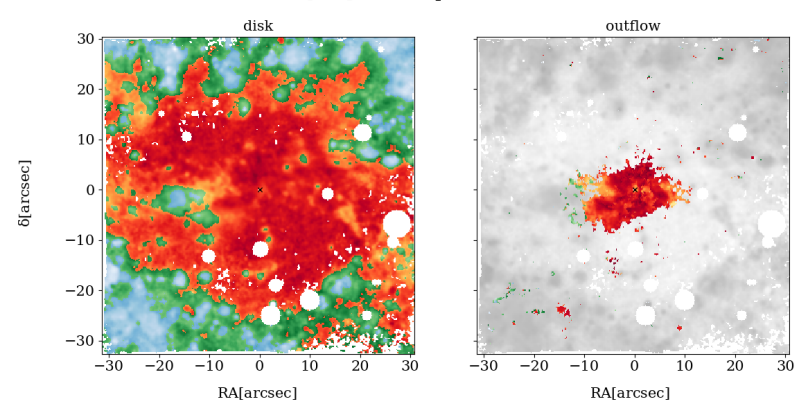

Fig. D.2. Same as Fig. D.1, but for NGC 1386, NGC 2992, NGC 4945, and NGC 5643. 
M. Mingozzi et al.: Gas properties in the outflowing and disc components in nearby active galaxies with MUSE
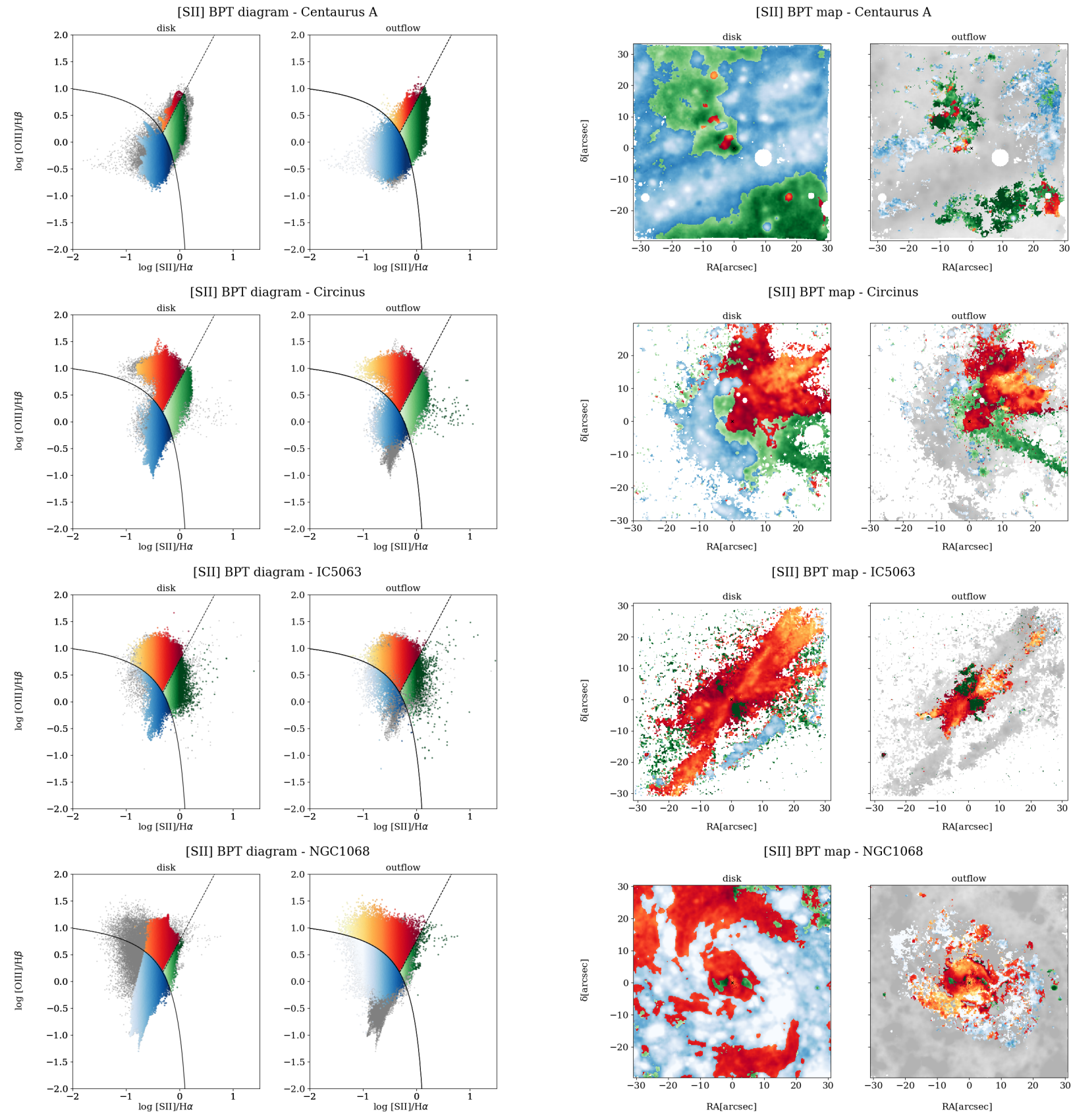

[SII] BPT map - NGC1068

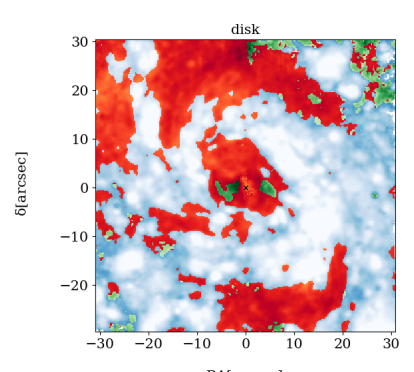

RA[arcsec]

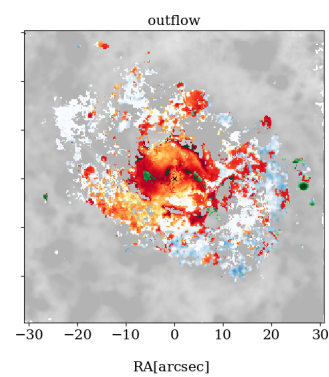

[SII] BPT map - NGC1365
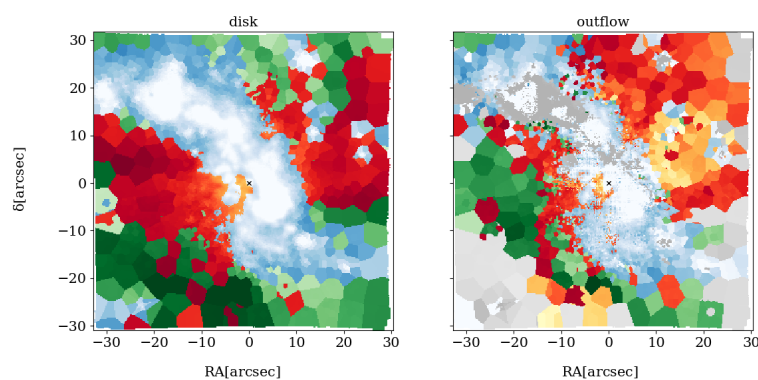

Fig. D.3. Left panels: [ $\left.\mathrm{S}_{\mathrm{II}}\right]$-BPT diagrams for the disc and outflow components, on the left and the right respectively, of Centaurus A, IC 5063, NGC 1068 and NGC 1365, colour-coded as follows: shades of blue for SF, green for LI(N)ER, and red for AGN-like ionising spectra, as a function of the $\left[\mathrm{S}_{\mathrm{II}}\right] / \mathrm{H} \alpha$ line ratio (darker shades means higher $\left[\mathrm{S}_{\mathrm{II}}\right] / \mathrm{H} \alpha$ ). The black solid curve is the theoretical upper limit on $\mathrm{SF}$ line ratios found by Kewley et al. (2001). The black dotted line is the Kewley et al. (2006) boundary between Seyferts and LI(N)ERs. Right panels: [S II]-BPT maps, colour-coded according to the BPT classification. In the background of all the pictures (black dots in the BPTs and shaded grey in the corresponding maps), are shown the disc and outflow component together. For each velocity bin, we select only the spaxels with a $S / N>5$ for all the flux line ratios. 

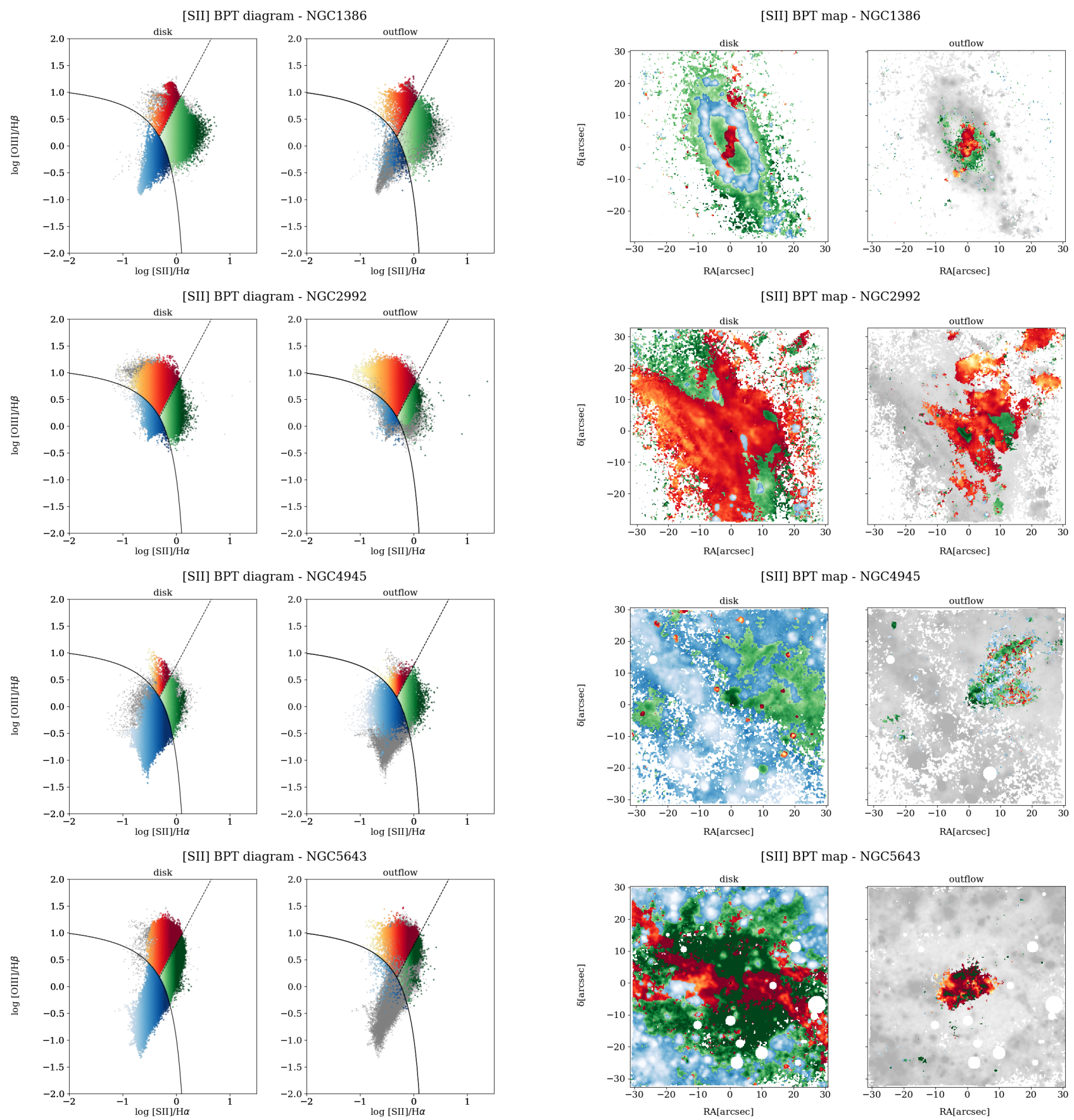

Fig. D.4. Same as Fig. D.3, but for NGC 1386, NGC 2992, NGC 4945 and NGC 5643. 
M. Mingozzi et al.: Gas properties in the outflowing and disc components in nearby active galaxies with MUSE

[NII] BPT diagram - Centaurus A

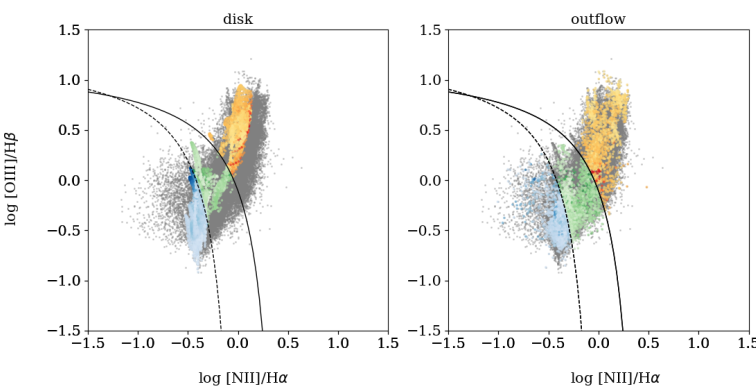

[NII] BPT diagram - IC5063
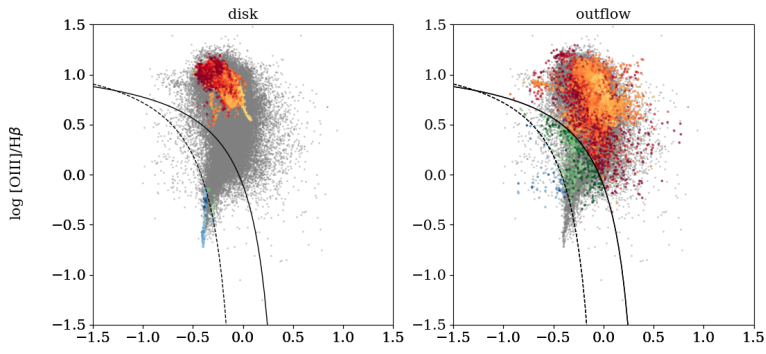

$\log [\mathrm{NII}] / \mathrm{H} \alpha$

[NII] BPT diagram - NGC1365
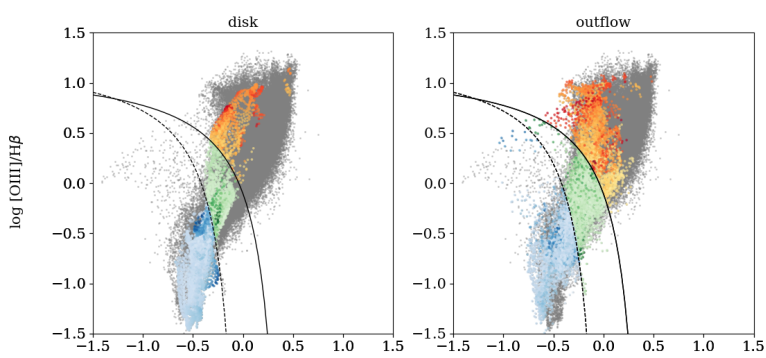

$\log [\mathrm{NII}] / \mathrm{H} \alpha$

[NII] BPT diagram - NGC2992

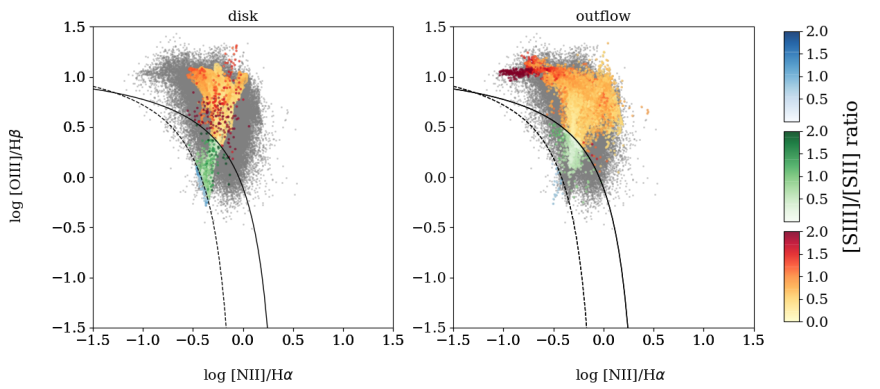

[NII] BPT diagram - Circinus

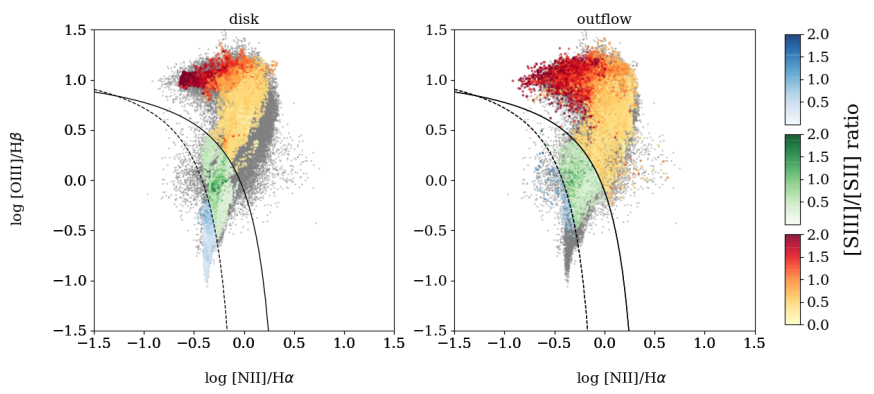

[NII] BPT diagram - NGC1068

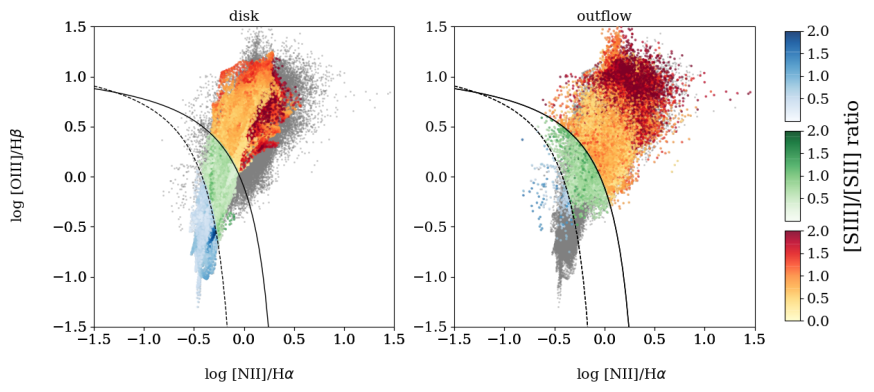

[NII] BPT diagram - NGC1386

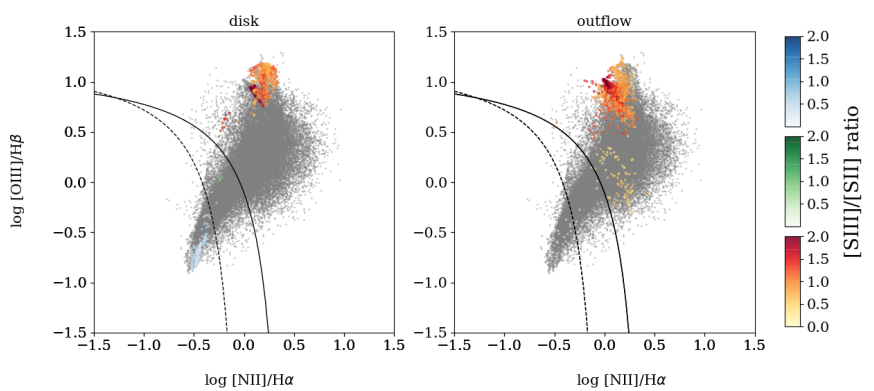

[NII] BPT diagram - NGC4945

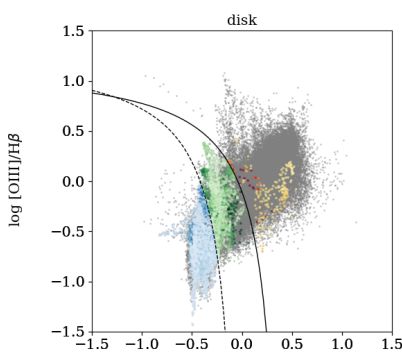

$\log [\mathrm{NII}] / \mathrm{H} \alpha$

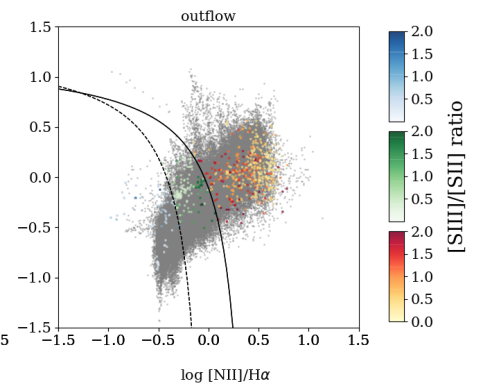

[NII] BPT diagram - NGC5643

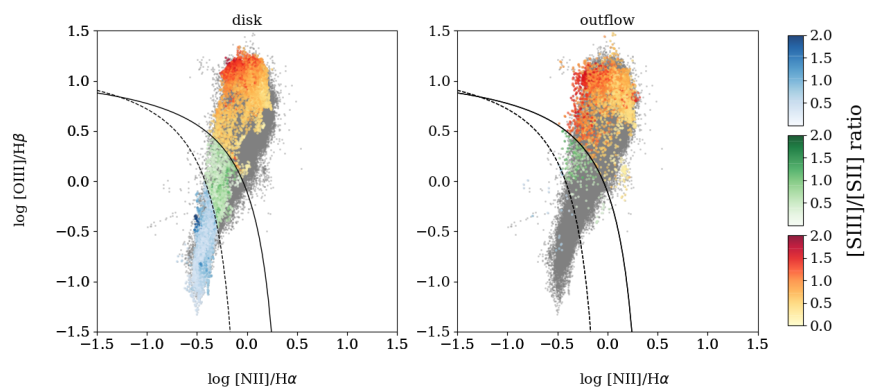

Fig. D.5. [N II]-BPT diagrams for the disc and outflow components for all the MAGNUM galaxies, colour-coded as a function of the [S III $] /\left[\mathrm{S}_{\mathrm{II}}\right]$ line ratio (darker shades means higher $\left[\mathrm{S}_{\mathrm{III}}\right] /\left[\mathrm{S}_{\mathrm{II}}\right]$ ). 

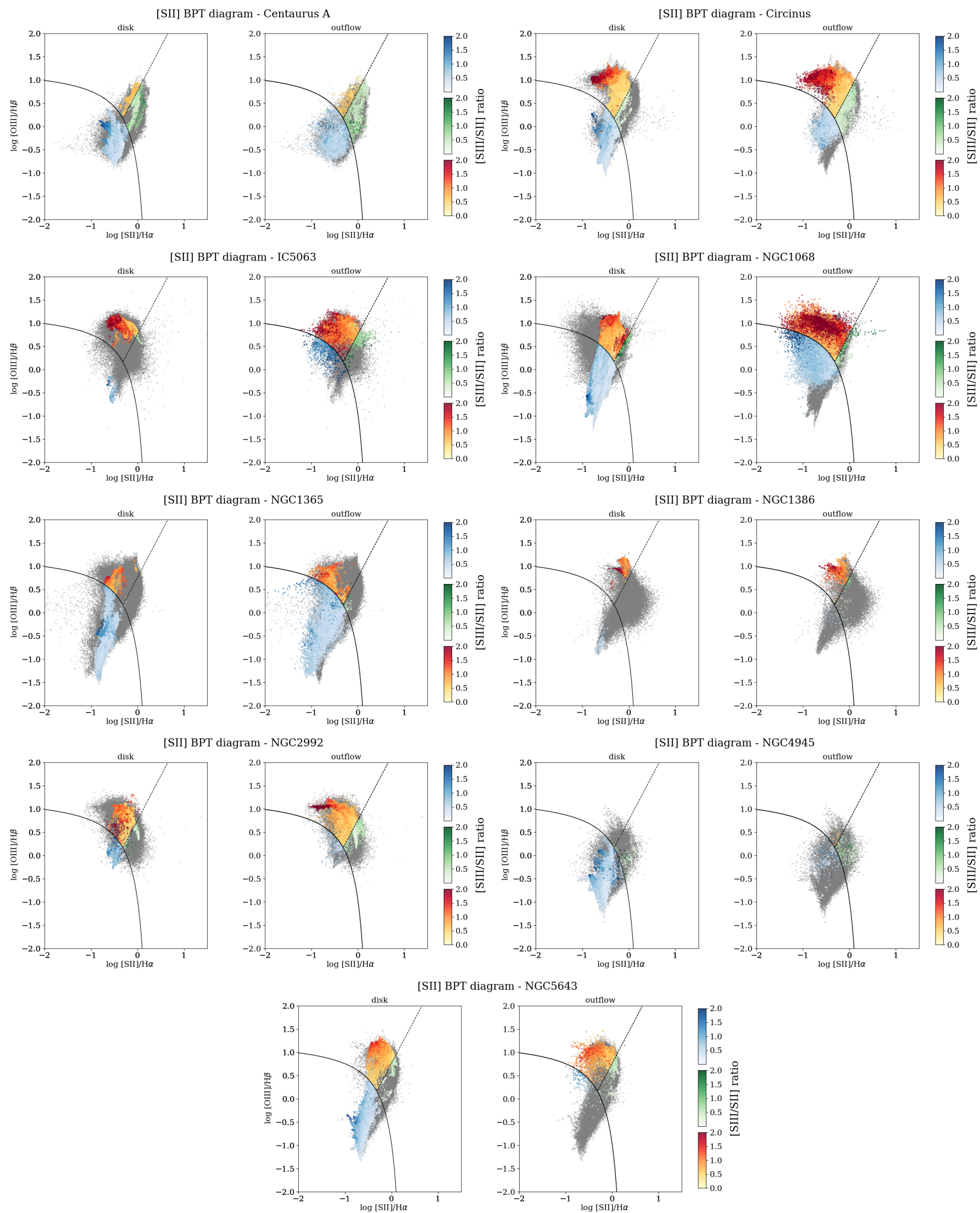

Fig. D.6. [S II]-BPT diagram for the disc and outflow components for all the MAGNUM galaxies, colour-coded as a function of the [S III]/[S II] line ratio (darker shades means higher $\left[\mathrm{S}_{\mathrm{III}}\right] /\left[\mathrm{S}_{\mathrm{II}}\right]$ ). 
M. Mingozzi et al.: Gas properties in the outflowing and disc components in nearby active galaxies with MUSE

[NII] BPT diagram - Centaurus A
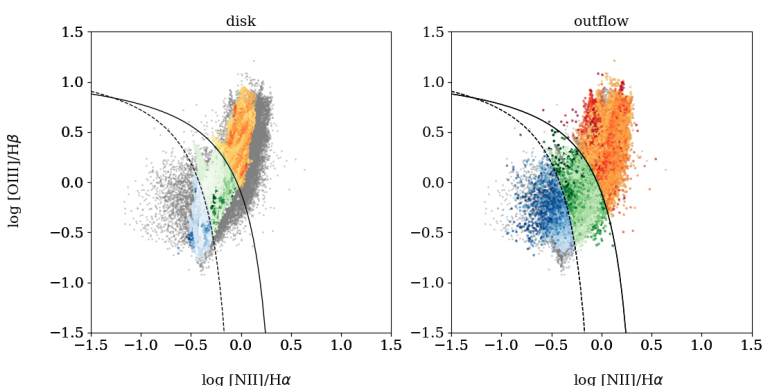

$\log [\mathrm{NII}] / \mathrm{H} \alpha$

[NII] BPT diagram - IC5063

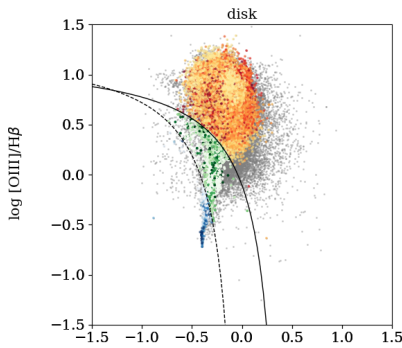

$\log [\mathrm{NII}] / \mathrm{H} \alpha$

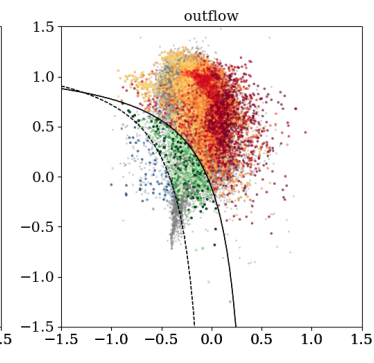

$\log [\mathrm{NII}] / \mathrm{H} \alpha$

[NII] BPT diagram - NGC1365
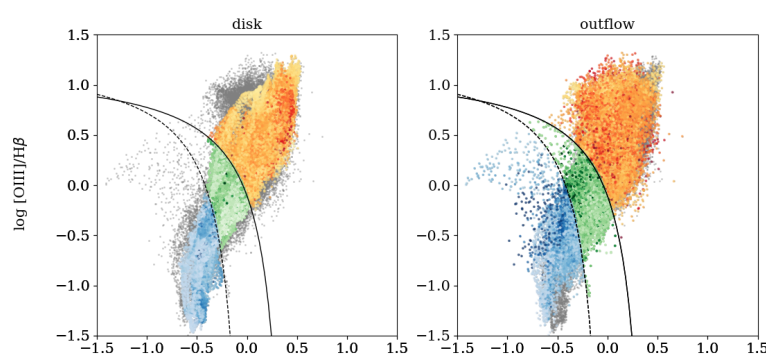

$\log [\mathrm{NII}] / \mathrm{H} \alpha$

[NII] BPT diagram - NGC2992

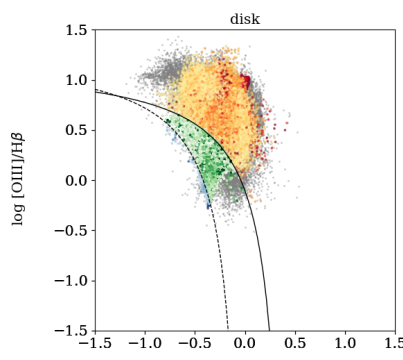

$\log [\mathrm{NII}] / \mathrm{H} \alpha$

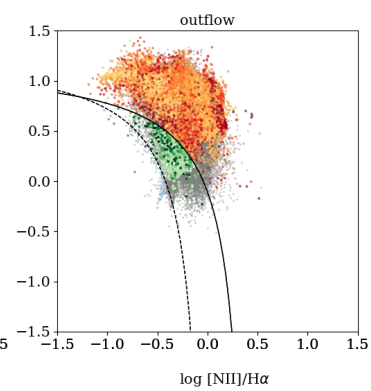

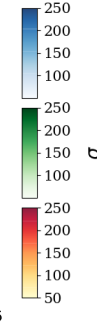
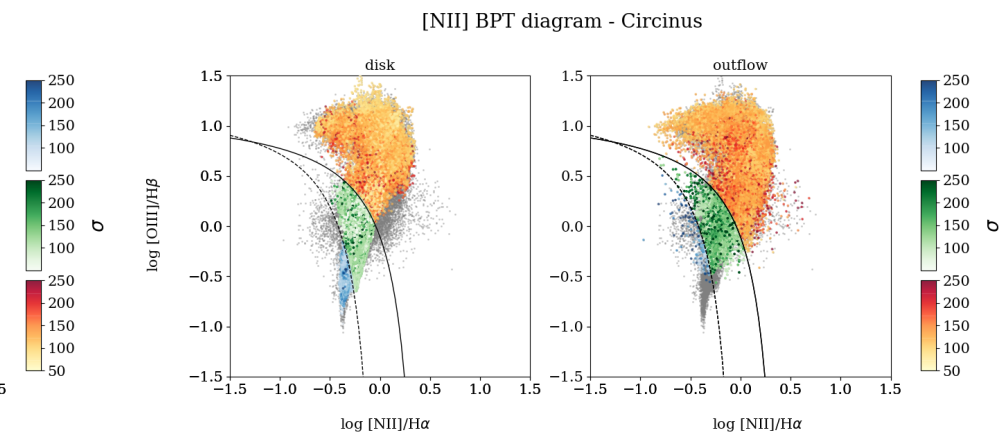

[NII] BPT diagram - NGC1068
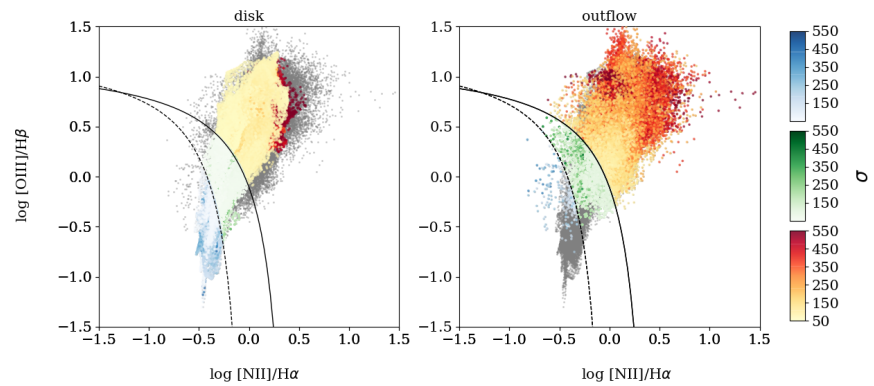

[NII] BPT diagram - NGC1386
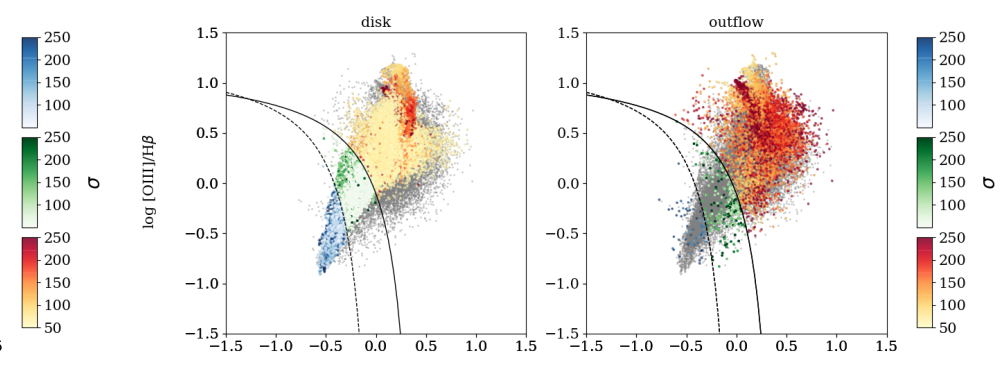

[NII] BPT diagram - NGC4945
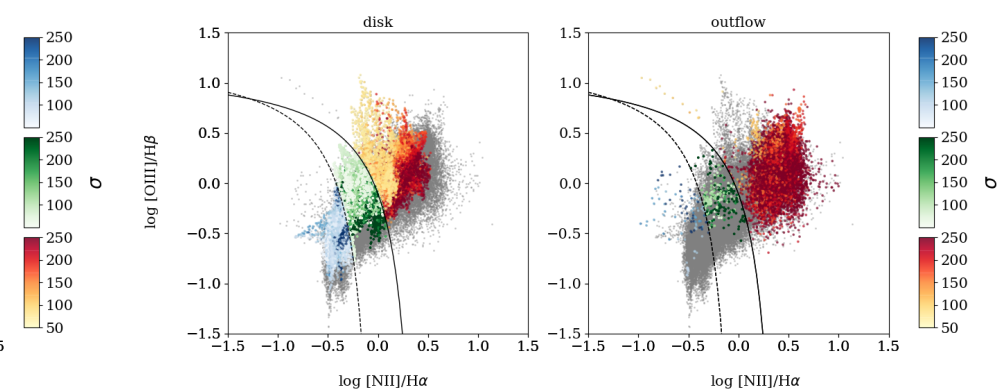

[NII] BPT diagram - NGC5643
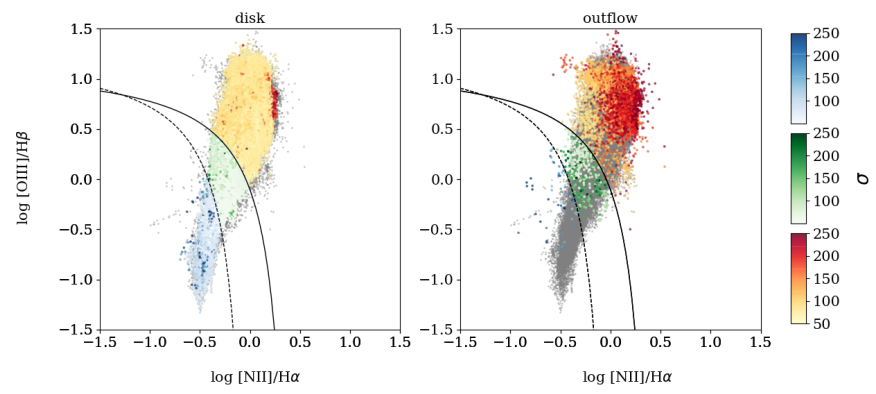

Fig. D.7. [N II]-BPT diagram for the disc and outflow components for all the MAGNUM galaxies, colour-coded as a function of the [O III] velocity dispersion $\sigma_{[\mathrm{OII}]}$ (darker shades means higher $\sigma_{[\mathrm{O} \mathrm{III}]}$ ). 

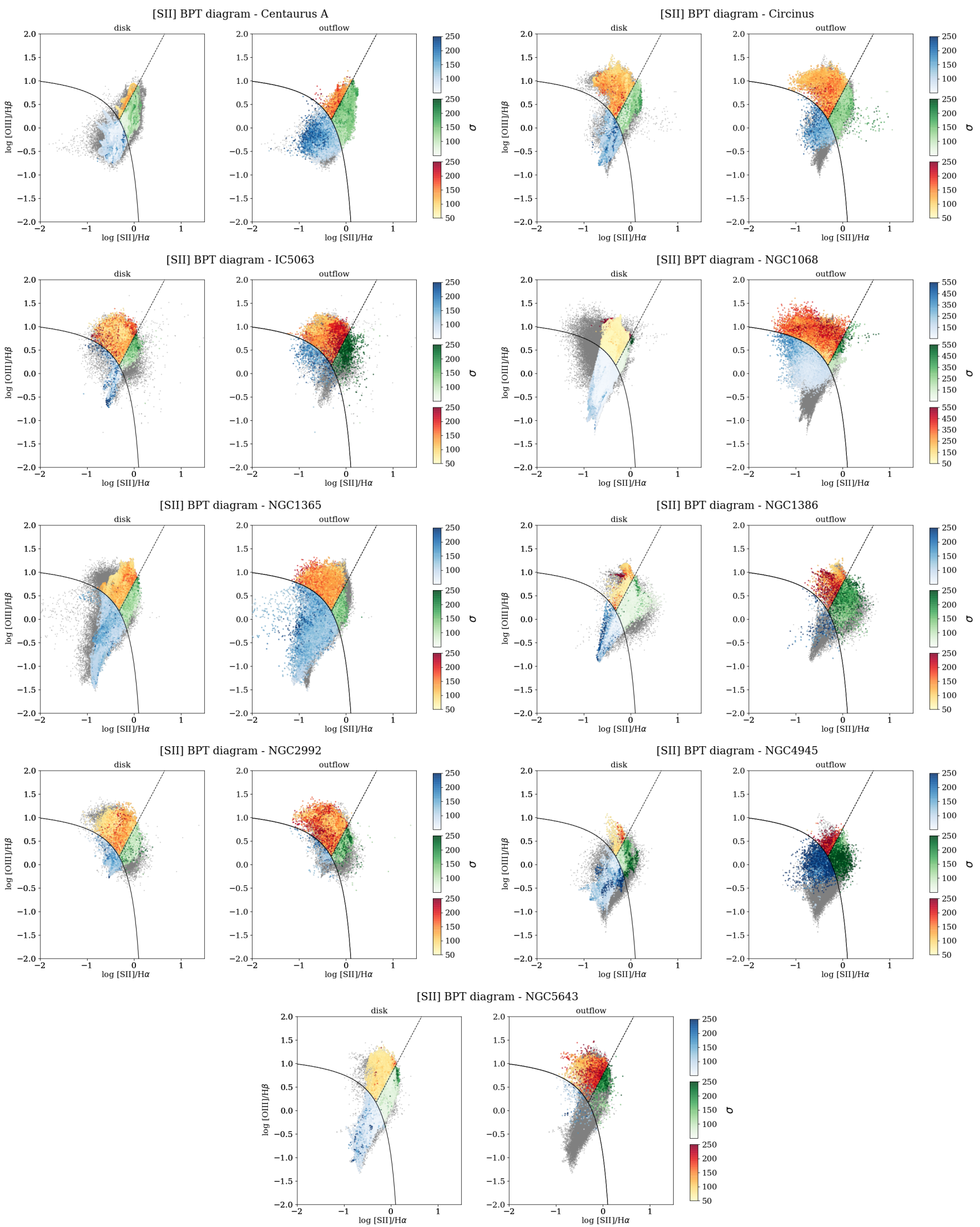

Fig. D.8. [S II]-BPT diagram for the disc and outflow components for all the MAGNUM galaxies, colour-coded as a function of the [O III] velocity dispersion $\sigma_{[\mathrm{OII}]}$ (darker shades means higher $\sigma_{[\mathrm{O} \mathrm{III}]}$ ). 